\title{
Establishment of an Animal Model of Depression: The Serotonin Transporter Knockout Rat
}

\author{
By \\ Meyrick Kidwell \\ A thesis submitted in fulfilment of the requirements of a Master of Science in Cognitive and Behavioural \\ Neuroscience
}





\section{Table of Contents}

Abstract

\section{Chapter One}

General Introduction

Major Depressive Disorder

Current Animal Models

Chapter Two

Conditioned Place Preference

Introduction

Method

Results

Discussion [26]

\section{Chapter Three}

Modified Successive Alleys Test

Introduction

Method

Results

Discussion 


\section{Chapter Four}

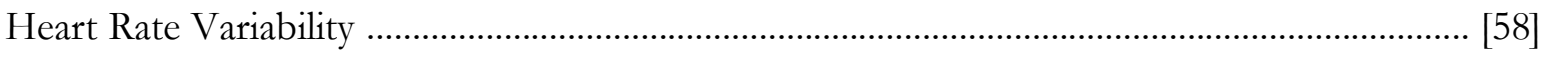

Introduction ……............................................................................................. [59]

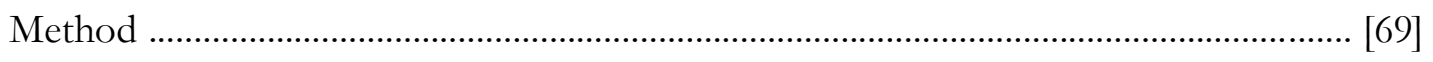

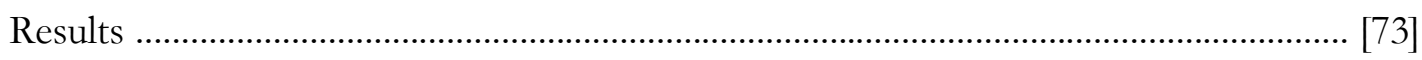

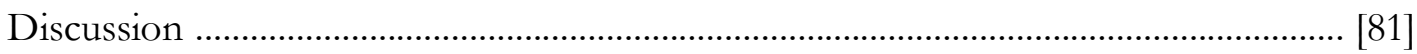

\section{Chapter Five}

Developmental Neurogenesis ............................................................................................. [88]

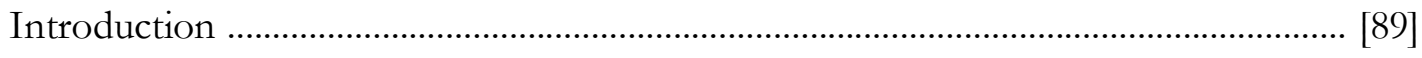

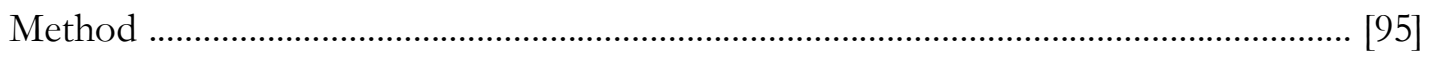

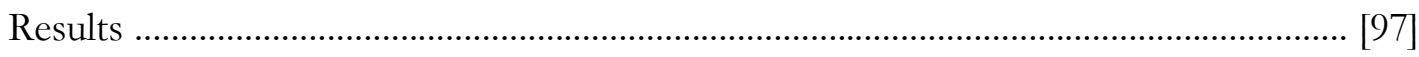

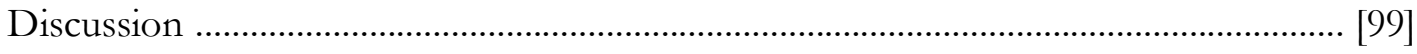

\section{Chapter Six}

Matrix Assisted Laser Desorption/Ionisation ....................................................................... [104]

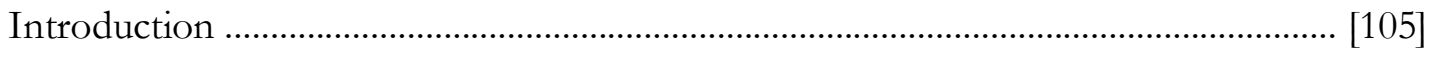

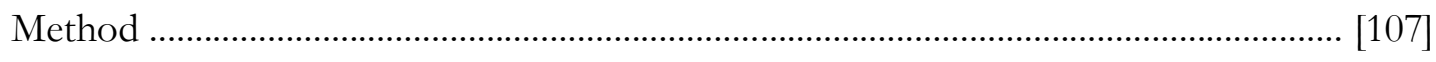

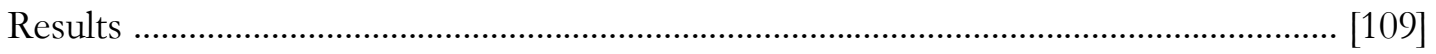

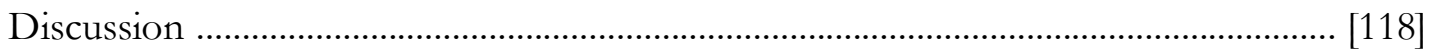




\section{Chapter Seven}

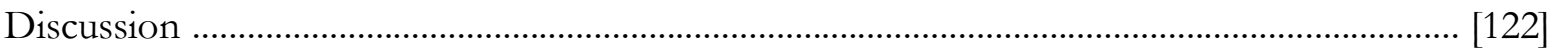

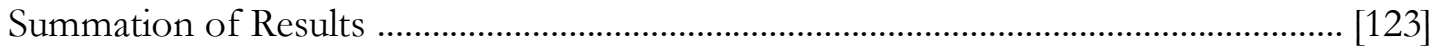

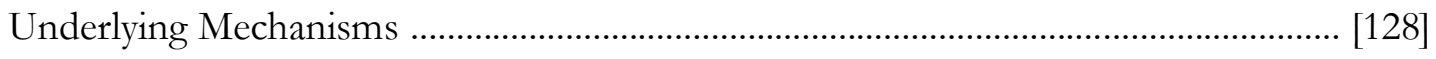

Translatability to Humans .............................................................................................. [130]

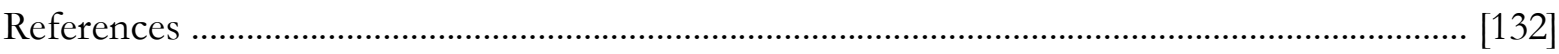

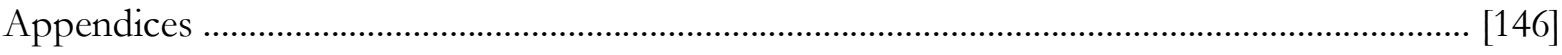


Major depressive disorder (MDD) is a serious and debilitating psychiatric illness found of increasing prevalence. Despite this, our current first line treatments have been shown to lack efficacy and possess a high non-response rate. Most new pharmacological developments have not shown efficacy in humans, likely due to our current models being outdated. This thesis attempts to use a range of novel approaches, integrating behavioural, physiological, and biological methods to provide support for the use of the serotonin transporter knockout (SERT-KO) rat to model components of MDD in humans. Social anhedonia is assessed through conditioned place preference and play behaviour analysis, demonstrating significantly reduced reward sensitivity in SERT-/- animals. Comorbid anxiety is assessed using a modified successive alleys test, whereby SERT-/- animals demonstrate increased anxiety behaviour, which persist over the course of the experiment. The assessment of heart rate variability, a physiological correlate of MDD was impacted by time constraints, however suggests a likely reduction to be present in the SERT-/animals. Finally, neurogenesis was found to be significantly increased in SERT-/- animals during early development (PND7), demonstrating neurodevelopmental alterations associated with reduced SERT expression. These data demonstrate that the SERT knockout rat possesses many deficits associated with MDD, thus being a likely candidate for novel pharmacological development. A final pilot experiment was conducted using MALDI-TOF to provide a method of examining potentially thousands of compounds in brain and cardiac tissue with high spatial definition. Applications and implication of this research are discussed in detail with suggestions for future studies being presented. 


\title{
CHAPTER ONE
}

\author{
Introduction
}

Our Current Understanding of Depressive Disorders and How

They are Modelled in Rodents 
Major Depressive Disorder (MDD) is characterized by a reduction in hedonic state (anhedonia), feelings of guilt or worthlessness, reduced motivation (avolition), fatigue, alongside disruptions in sleep and appetite (Association, 2013). Between 2005 and 2015, prevalence rates of depression increased by $18.4 \%$ (Hay et al., 2017) with current prevalence estimates placing depression as the leading cause of disability worldwide (Organization, 2017). In severe cases, depression can lead to suicide which is the second leading cause of death in those aged 15-29. Despite the severity and increasing prevalence rates of depression, treatments still focus on the monoamine hypothesis of the disease which posits that an imbalance in monoamines such as serotonin, noradrenaline, and dopamine underlie the disease (Krishnan \& Nestler, 2008). While this approach has been useful in the development of pharmaceutical interventions, their effectiveness remains relatively low (Locher et al., 2017; Pigott, Leventhal, Alter, \& Boren, 2010), and the delayed onset of effectiveness is linked to several significant limitations including increased suicidality in initial stages of treatment (Fergusson et al., 2005) and reduced compliance (Keller, Hirschfeld, Demyttenaere, \& Baldwin, 2002).

While the high prevalence of MDD and the concurrent impact of the disorder, and its comorbidities are well documented, there have been few attempts to investigate the long-term impacts outside the symptomology of the disorder when left untreated, or when treatments prove unsuccessful. A recent study by Setiawan et al. (2018) demonstrated that untreated depression can result in progressive neurodegeneration related to the duration of the illness, demonstrating the urgent need for novel treatments of MDD.

To develop novel pharmacological interventions, there is a need for new animal models of depression which closely align with the current research-based understanding of the disorder. Most attempts to model depression are based on the (usually repeated) exposure to stressor, as depression is often regarded as a stress related disorder (Caspi et al., 2003). For example, one widely used model that involves the induction of stress to produce depressive behaviours is the 
chronic mild stress model, which through the exposure to unpredictable long term stressful stimuli, the animal exhibits depressive traits (Willner, Towell, Sampson, Sophokleous, \& Muscat, 1987). This model has become popular as it shows high predictive validity as chronic antidepressant treatments alleviate the depressive symptoms such as anhedonia. Further, the CMS model has been shown to produce similar neurobiological deficits observed in depressed humans (Willner, 2005). While CMS is a strong candidate for modelling depression, and has produced developments within drug discovery, it should be noted that this model is labour intensive. Further, these behavioural deficits are often transient in nature (Grippo, Beltz, \& Johnson, 2003; Pothion, Bizot, Trovero, \& Belzung, 2004), thus not being clearly representative of a chronic disorder such as MDD, rather, CMS may be a better representative of situational depression. More recently, the social defeat model (often referred to as the resident-intruder test) has gained popularity as a model of depression (Hollis \& Kabbaj, 2014; Keeney \& Hogg, 1999). Through introducing a target animal into the home cage of an older, more aggressive male, the target animal is forced into submission, inducing a strong physiological and emotional stress response (Tornatzky \& Miczek, 1993). Through multiple exposures to social defeat, a number of behavioural changes including social avoidance and anhedonia have been demonstrated (Hollis \& Kabbaj, 2014; Rygula et al., 2005) while biological alterations such as reduced neurogenesis, mobility, and food consumption have been noted (Becker et al., 2008). Importantly, it should be noted that these deficits can be reversed through chronic antidepressant treatment.

While the social defeat model of depression appears to be a strong candidate, it suffers from some unique and some similar flaws as the CMS model; Firstly, sucrose preference has been shown to be not affected by repeated social defeat (Hollis \& Kabbaj, 2014). Given the use of sucrose preference as a well-established measure of stress induced anhedonia (Hollis, Wang, Dietz, Gunjan, \& Kabbaj, 2010; Willner et al., 1987), the proposed induction of depressive behaviours should be interpreted with caution. Secondly, as with the CMS model, some of the induced depressive effects are transient in nature, including reduced BDNF expression, body weight 
reductions, and despair behaviours, and exploration (de Jong, van der Vegt, Buwalda, \& Koolhaas, 2005; Fanous, Hammer Jr, \& Nikulina, 2010; Krishnan et al., 2007). These transient effects do not mimic the human depressive experience of persistent dysfunctional behaviours and low mood, thus the social defeat model is more clearly modelling situational depression. Moreover, it is well established that MDD has a strong genetic component (Lopez-Leon et al., 2008) which is not incorporated in such stress models, likely explaining the transient effects previously described.

Another limitation of the current generation of animal models for MDD is the assessment of MDD-like symptoms. While there are many behavioural assays used to assess depressive-like behaviours, one of the most commonly used is the forced swim test (FST). The FST is thought to model behavioural despair, measured through immobility in an inescapable chamber of water, believed to reflect depressive tendencies. That is, the greater the proportion of time spent immobile, is indicative of greater depressive behaviour. Importantly, this assumption was initially developed due to the observation that through administration of antidepressants, immobility is reduced, thus indicating the reduction of depressive behaviours (Porsolt, Le Pichon, \& Jalfre, 1977). While the FST has been shown to be reasonably predictive of antidepressant efficacy through reduced immobility, it would be fallacious to assume that increased immobility is predictive of increased depressive-like behaviours. Furthermore, acute antidepressant administration reduces immobility, however, chronic administration is required to reduce depressive symptoms in humans. This suggests that the mechanisms underlying the antidepressant response in the FST likely differ from those underlying the reduction of depressive symptoms in response to antidepressants in humans (Borsini, Volterra, \& Meli, 1986). Thus, in addition to more faithfully mimicking the aetiology of MDD, better assays for assessing MDD like features are equally warranted.

In the present thesis we have therefore aimed to develop a model of MDD through genetic manipulation, by introducing a mutation which has often been associated with this disorder in 
humans. Specifically, we focussed on a common polymorphism (5HT'TLPR, 5-HT transporter linked polymorphic region) in the gene (SLC6A4) which encodes for the serotonin transporter (SERT). This polymorphism consists of a 44 base pair insertion/deletion ( 1 allelic variant $\&$ s allelic variant respectively) (See figure 1), resulting in a 50\% reduction in SERT activity in the ss-genotype, compared to the ll-genotype, which in turn enhances extracellular levels of serotonin in the synaptic cleft (Nakamura, Ueno, Sano, \& Tanabe, 2000). Due to the widespread effects of serotonin through development and adulthood, the increased extracellular serotonin as a result of this mutation has been associated with several neuropsychiatric disorders, but most predominantly with MDD (Collier et al., 1996; Serretti et al., 2002).

Figure 1.

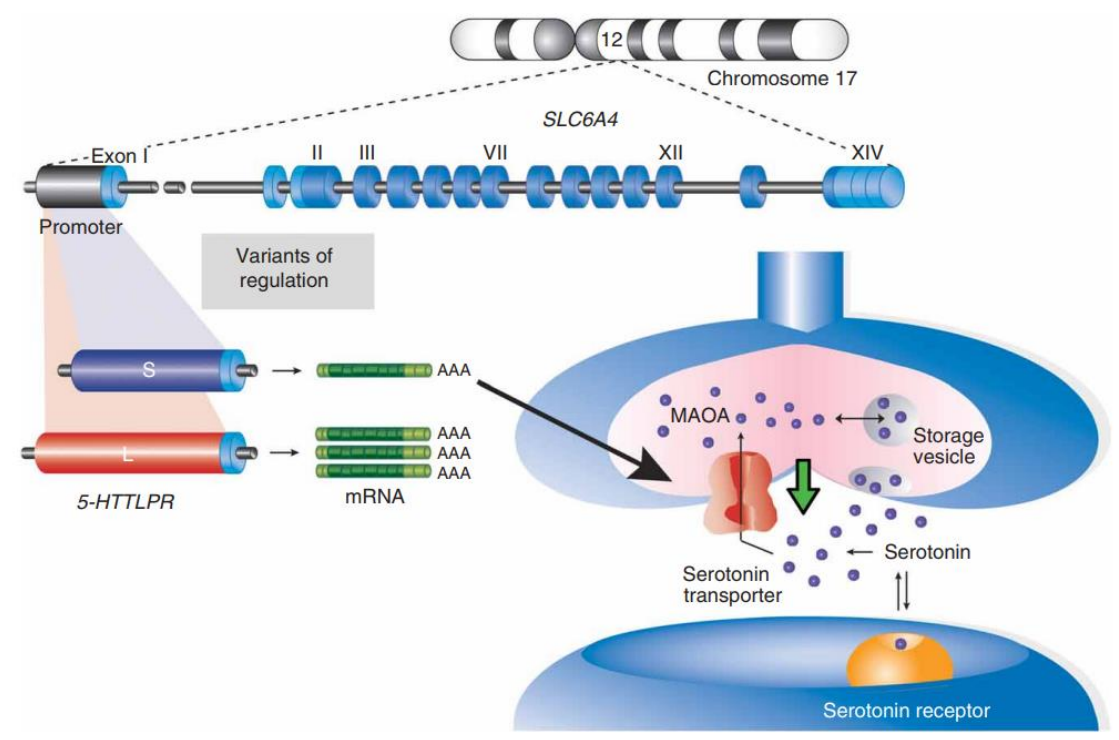

Figure 1. Visual depiction of the common polymorphism (5HTTLPR, 5-HT transporter linked polymorphic region) in the SLC6A4 gene which encodes for the serotonin transporter. A 44 base pair deletion is depicted in purple (s allelic variant), while the 44 base pair insertion is depicted in red (l allelic variant).

While this mutation does not naturally occur in rodents, advancements in genetic manipulation techniques have allowed researchers to reproduce this down regulation of the 
serotonin transporter expression in a laboratory rat strain through $\mathrm{N}$-ethyl-N-nitrosourea (ENU mutagenesis) (Smits et al., 2006). Following the development of this mutant rat, referred to as the SERT knockout (Slc6a4 ${ }^{1 \text { Hubr }}$ ) rat, J. Homberg et al. (2007), examined the impact of this mutation by comparing SERT knockouts, both homozygous $\left(\mathrm{SERT}^{-/}\right)$and heterozygous $\left(\mathrm{SERT}^{+/}\right)$, with those not expressing this mutation $\left(\mathrm{SERT}^{+/+}\right)$. The results indicate that there is a gene dose dependant reduction of the SERT levels, that is a 100\%, and 50\% reduction in SERT knockouts $\left(\mathrm{SERT}^{-/-}\right.$and $\mathrm{SERT}^{+/-}$compared to $\mathrm{SERT}^{+/+}$respectively). Furthermore, there was a nine-fold increase in extracellular concentrations of serotonin in the hippocampal region of SERT ${ }^{-/-}$animals. Finally, the authors determined that no compensatory mechanisms occurred in tryptophan hydroxylase (serotonin synthesizing enzyme) or monoamine oxidase (serotonin metabolizing enzyme). Moreover, no major changes in other monoamines, most notably dopamine and noradrenaline, were found.

Since the development of the SERT knockout rat, it has been subjected to numerous behavioural assays to examine the behaviour consequence of the genetic reduction of the serotonin transporter. Neumann et al. (2011) summarised the deficits observed in SERT knock out rats, with the overview of the data strongly suggesting the validity of this strain as a model for anxiety disorders due to the increased anxiety-like behaviour in a variety of behavioural paradigms, reduced social behaviours, HPA axis dysregulation, and increased amygdala responsiveness. However, it should be noted that the behavioural abnormalities are not limited to modelling anxiety, rather, SERT knockout rodents have also exhibited behaviours frequently associated with depression, including reduced sucrose preference, and increased immobility in the FST (J. Olivier et al., 2008).

This thesis seeks to further explore the impact of the genetic reduction in the expression of SERT as an animal model for depression, using several new techniques. By integrating behavioural, neurobiological, and physiological approaches we hope to further increase our understanding of the validity of the SERT knockout rat as translational model of MDD. 


\title{
CHAPTER TWO
}

\author{
Experiment One
}

Conditioned Place Preference and Social Behaviour 
Humans, like many mammalian species, are innately social beings with theories suggesting that sociability evolved to improve survivability against predation, improve resource collection, and improve gene pools for mate selection (Alexander, 1974). While social interaction and behaviour in itself has evolved over time, it undeniably remains one of the core elements behind the progression and expansion of the human species. The influential hierarchy of needs models propose that the requirement of social belonging falls after the acquisition of physiological and safety requirements (Maslow, 1943), whereas more recent theories propose the requirement of sociability to fall alongside these basic requirements as opposed to the linear hierarchy of needs model (Alderfer, 1969). Nonetheless, given the importance of social interactions in humans, reductions in social behaviours associated with psychiatric conditions are troubling and significantly impact the overall burden of disease. Reductions in social interest and interactions are also frequently reported in Major Depressive Disorder (MDD) (Beck, Steer, \& Brown, 1996; Hamilton, 1960).

To highlight the importance of social relationships in depression, several studies have investigated the detrimental impacts of negative social interactions and social isolation on the development and maintenance of MDD. A longitudinal study examining the social predictors of depression after a 10-year follow up $(\mathrm{n}=4642)$ found that lack of social support, greater social strain, and poorer social relationship quality were all predictive of greater depression scores after 10 years (Teo, Choi, \& Valenstein, 2013). Further longitudinal evidence implicates depressive symptoms in the future reduction of social support (Stice, Ragan, \& Randall, 2004), which suggests a bidirectional relationship between social relationships and depressive symptoms. Additionally, and of greatest concern is that preference for solitude combined with social isolation is predictive of suicidal ideation and self-harming behaviours (Endo et al., 2017). Furthermore, the act of social withdrawal and isolation was shown to frequently precede a suicide attempt (Baumeister, 1990; Stravynski \& Boyer, 2001). Given these relationships, the understanding of social behaviours should be examined further and integrated into new depression models. 
While the reduction in social interactions in MDD is relatively well documented, it should be noted that the relationship may be more complex than first thought. One study examined a community sample to investigate the relationship between social interactions and depressive symptomology (Nezlek, Hampton, \& Shean, 2000); half of those studied met the criteria for clinical depression, these participants did not differ in the amount of day-to-day social interaction reported when compared to the rest of the sample. However, differences emerged when investigating the enjoyment rating of these interactions. Depressed participants rated the interactions as less enjoyable and felt they had less control over these interactions. In other words, depressed individuals may experience more social anhedonia, which in turn may precede social withdrawal observed in those with (more severe) depression.

Serotonin has been strongly implicated in depressive disorders, particularly, in regards to the social component of behaviour (S. N. Young \& Leyton, 2002). For example, increased serotonin is related to improvements in prosocial behaviours such as cooperation and bonding, while conversely, reductions in serotonin, are associated with reduced prosocial behaviours and increased antisocial behaviours such as aggression (Duke, Bègue, Bell, \& Eisenlohr-Moul, 2013; Kiser, SteemerS, Branchi, \& Homberg, 2012; Kruesi et al., 1990). To examine the importance of the serotonergic system, a frequently used method is to reduce tryptophan levels through the acute tryptophan depletion method (ATD) (Ellenbogen, Young, Dean, Palmour, \& Benkelfat, 1996). Through this method the participant ingests food devoid of tryptophan, which subsequently leads to a reduction in synthesised serotonin. Using this method, it has been demonstrated that an acute reduction in serotonin results in significant reductions in mood and social interaction (S. N. Young \& Leyton, 2002). Conversely, by selectively increasing serotonergic activity, we would expect elevations in mood and social interactions. One such method of increasing extracellular serotonin is through 3, 4-Methylenedioxymethamphetamine (MDMA) which is a widely used recreational drug which has anecdotally been reported to improve mood and social connectedness which underlie its popularity (Vollenweider, Liechti, Gamma, Greer, \& Geyer, 2002). Further studies 
have examined these effects to support the anecdotal reports suggesting that MDMA administration improves mood and facilitates social communication (Brothers, 1996). Based on these findings, some have even suggested that MDMA administration may assist in therapeutic psychotherapy settings by acting as a catalyst to enhance therapist-patient bonding and improve communication (Oehen, Traber, Widmer, \& Schnyder, 2013; Sessa, 2007).

Current treatments of mood disorders primarily involve modulation of the serotonin system through selective serotonin reuptake inhibitors (SSRIs), which increase extracellular serotonin levels by preventing the transportation out of the synapse. Studies examining the impact of antidepressant medication on social symptoms of mood disorders has been mostly positive, indicating that through increasing synaptic serotonin levels, elevations in prosocial behaviours occur, while antisocial behaviours tend to be reduced (Bond, 2005; Carrillo, Ricci, Coppersmith, \& Melloni, 2009; Kiser et al., 2012; Steenbergen, Jongkees, Sellaro, \& Colzato, 2016). For example, New et al. (2004) sought to examine the impact of fluoxetine (an SSRI) on impulsive aggression in patients who had exhibited aggressive behaviours. The results of this study showed significant clinical improvements in aggression, thus indicating the regulation of antisocial type behaviour through alterations in the serotonergic system. Further supporting the importance of serotonin in regards to prosocial behaviour, Harmer et al. (2003) examined the impact of acute administration of the SSRI citalopram on the processing of social information through a facial expression task. Those in the citalopram group showed greater accuracy in detecting happy and fearful expressions at a faster rate when compared to the placebo control group. This indicates that citalopram improves the rate and accuracy at which social information is processed.

Genetic evidence also links serotonin to social behaviour. Thus, carriers of the $1 / 1$ genotype of the 5-HTTLPR demonstrated better social adjustment scores in relation to work and family when compared to carriers of the s allele. Additionally, the s-allele has not only been linked to seasonal affective disorder per se (Rosenthal et al., 1998), but particularly to social deficits in this 
disorder. Stoltenberg, Christ, and Carlo (2013) sought to investigate the underlying factors involved in the relationship between the 5-HTTLPR polymorphism and prosocial behaviours. Using a self-report questionnaire, the authors found prosocial behaviours to be increased in homozygous 1 allele carriers while agoraphobia and social phobia were increased in s allele carriers. Furthermore, they found that social phobia partially mediated the relationship between 5HTTLPR and prosocial behaviours indicating that s-allele carriers are less likely to engage in prosocial behaviours in part due to their anxiety surrounding social engagement. While the majority of studies have since focussed on behavioural and psychological data, there also exists a vast literature on the neuronal impacts of the 5-HTTLPR polymorphism. Munafò, Brown, and Hariri (2008) conducted a meta-analysis (14 studies, $n=499)$ regarding the 5-HTTLPR polymorphism and amygdala activation, a core structure involved in emotion, emotional recognition, and social behaviour. Amygdala activity was measured primarily through functional magnetic resonance imaging (fMRI), with two studies using perfusion magnetic resonance imaging. This meta-analysis showed significantly heightened amygdala activity in response to emotional stimuli in s-allele carriers. Furthermore, Canli and Lesch (2007), using a similar approach found that the 5-HTTLPR polymorphism underlies social cognition, and emotion regulation.

Given the role of the 5-HTT gene in social behaviour in humans, the SERT knock out (Smits et al., 2006) rats provide a prime candidate to explore these behaviours further in a controlled experimental environment. Due to this isolated genetic mutation, the use of this animal model in the study of social behaviour allows for the examination of the gene-specific contributions to social behaviour. To date, few studies have examined the social behaviours of SERT knockout animals. Moy et al. (2009) examined socialization behaviour in SERT knockout mice through a two-chamber test. One chamber contained an unfamiliar caged animal, while the other contained no stimulus. SERT ${ }^{-/-}$male mice spent significantly less time investigating the unfamiliar mouse compared to $\mathrm{SERT}^{+/+}$and $\mathrm{SERT}^{+/-}$mice. Furthermore, the time spent in the empty chamber was marginally greater than that spent in the chamber with the other animals. This 
could suggest an aversion to socialization, however, this measure of social behaviour is relatively crude and difficult to tease apart separate elements of social behaviour. In contrast, Homberg, Schiepers, Schoffelmeer, Cuppen, and Vanderschuren (2007) conducted a detailed analysis of social behaviours in peri-adolescent rats (post-natal days 28-35). Animals were placed in pairs into the experimental arena and allowed to freely interact for 15 minutes. The SERT ${ }^{-/}$group had significant reductions across all social play behaviours (such as pinning, pouncing and wrestling, however, non-playful social interactions (such as social grooming) remained unaffected, thus implicating the genetic reduction of serotonin transporter expression in social play behaviours, but not non-playful interactions. While this study remains to be one of the most comprehensive analyses of the serotonin transporters contribution to deficits in social behaviour, it is in urgent need of replication.

Moreover, it is unclear why play behaviour, but not non-play social behaviour is reduced. As previously mentioned, a reduction in reward from social interaction may partially explain reductions in social behaviour. The SERT knockout animals have been shown to have a reduced reward sensitivity and have been shown to exhibit anhedonia. For example, J. Olivier et al. (2008) examined the impact of the SERT knockout mutation on sucrose consumption (a crude but robust measure of anhedonia and reward sensitivity). SERT ${ }^{-/}$animals were found to have a reduction in sucrose consumption, with this deficit becoming more prominent as sucrose concentration increased. These results indicate a reduction in reward sensitivity suggesting the presence of anhedonia. Furthermore, these deficits have been observed in other paradigms including a food reinforced progressive responding schedule (Sanders, Hussain, Hen, \& Zhuang, 2007), and delayed based decision making (Denk et al., 2005). However, SERT downregulation did not affect effort based learning (Denk et al., 2005) and increased sensitivity to drug induced rewards caused by cocaine and MDMA administration have been observed (Homberg et al., 2008; Oakly, Brox, Schenk, \& Ellenbroek, 2014). Thus, while differences in avolition and anhedonia are present in the SERT knockout rat, they are not uniformly observed. Thus, it is important to establish whether 
SERT knockout impacts social reward similarly to food reward, or whether an alternative mechanism may be underlying this association.

One method which has classically been used to assess the rewarding properties of drugs of abuse is the conditioned place preference (CPP) paradigm (Tzschentke, 1998), which, has also been adapted to assess the rewarding properties of social interaction (Trezza, Damsteegt, \& Vanderschuren, 2009). In this paradigm, animals are placed in one of two chambers. Classically, the animal will be infused with a drug with rewarding properties when placed into one chamber, while, no rewarding stimulus is given before the animals are placed in the second chamber. Following the conditioning phases, an animal will spend more time in the drug-paired chamber when allowed to freely choose between chambers. To assess social reward, animals are placed into one chamber with another animal and allowed to interact (or alone in the other chamber). Due to the social nature of rats, being paired with another rat acts as the rewarding stimulus, thus inducing a similar preference for the conditioned chamber in the assessment phase as to what is seen with drugs of abuse (Tzschentke, 2007). Douglas, Varlinskaya, and Spear (2004) provided a detailed assessment of factors likely to induce the greatest preference development. Their results showed that peri-adolescent rats which have been isolated for at least one week prior to conditioning and exposed to 10-minute interaction sessions will develop the greatest preference.

To determine whether the reduction in serotonin transporter expression reduced reward sensitivity for social stimuli, $\mathrm{SERT}^{+/+}, \mathrm{SERT}^{+/}$, and $\mathrm{SERT}^{-/}$rats were examined using the social CPP paradigm. Based on the previously discussed literature, we hypothesised a gene-dose reduction in social place preference. That is, the $\mathrm{SERT}^{+/+}$group would show the largest increase in preference for the socially paired chamber when compared to the $\mathrm{SERT}^{+/-}$and $\mathrm{SERT}^{-/}$rats. 


\section{Method}

Subjects

A total of 32 male Wistar rats were used for this experiment consisting of $10 \mathrm{SERT}^{+/+}, 12$ SERT $^{+/}$, and 10 SERT $^{-/-}$animals (Smits et al., 2006). All subjects were weened at PND 21 from $\mathrm{SERT}^{+/-}$dams and placed directly into individual housing for the remainder of the duration of this experiment. Tissue samples from an ear clipping were sent to be genotyped (Transnetyx, Cordova US), while, subcutaneous RFID implants were injected for later identification. Social isolation was intended to last for 3-5 days prior to the commencement of the experiment, however, due to delays in receiving the genotyping results, this period had to be extended to 10 days, placing the animals in the middle of the peri-adolescent stage of development (PND31) at the beginning of the experiment. All rats were singularly housed in polycarbonate cages in a temperature and humidity (55-60\%) controlled environment, $21^{\circ} \mathrm{C} \pm 2$ and 55-60\% respectively. Animals were housed under a reverse light cycle (lights on at 1900-0700 hours) to ensure testing occurred during active periods. Animals were bred and housed in the Victoria University of Wellington vivarium in accordance with the Animal Welfare Act 1999, with all procedures being approved by the VUW Animal Ethics committee (AEC number 24384).

\section{Apparatus}

Plexiglas locomotor activity boxes (Med Associates Inc, USA; model ENV-515) with the dimensions of $42 \times 42 \times 30 \mathrm{~cm}$ were contained within closable cabinets. Further, two chamber CPP inserts with two compartments (Med Associates Inc, USA; model ENV-517) were fitted into each box to create two clearly distinguishable compartments (table 1). The left compartment contained a grid mesh floor with high contrast black and white vertical stripe wall art, while the right compartment contained bar flooring with high contrast black and white circle pattern wall art figure 1). A fixed divider wall with a sealable passage in the centre separated each compartment. Infrared beams were located across the walls to monitor the rat's movements using a standard beam break 
protocol, allowing accurate detection of the subjects positioning. Med Associates computer software collected data from the locomotor activity boxes, which was then processed for analysis. Infrared cameras were installed directly above the locomotor activity boxes using an adjustable mount to record the subjects' behaviours.

Table 1.

Dimensions and construction of the CPP insert (Med Associates Inc, USA; model ENV-515)

\begin{tabular}{l|ccc}
\hline \hline & \multicolumn{3}{c}{ CPP Apparatus (Med Associates Inc, USA; model ENV-515) } \\
\hline \multicolumn{1}{c}{ Total } & Paired Chamber & Unpaired Chamber \\
\hline Width & $42 \mathrm{~cm}$ & $20.5 \mathrm{~cm}$ & $20.5 \mathrm{~cm}$ \\
Length & $42 \mathrm{~cm}$ & $42 \mathrm{~cm}$ & $42 \mathrm{~cm}$ \\
Height & $30 \mathrm{~cm}$ & $30 \mathrm{~cm}$ & $30 \mathrm{~cm}$ \\
Visual Cue & N/A & High Contrast Circles & High Contrast Stripes \\
Tactile Cue & N/A & Bar Flooring & Grid Flooring \\
\hline
\end{tabular}

Figure 1. A three-dimensional visual depiction of the CPP apparatus used in the present experiment

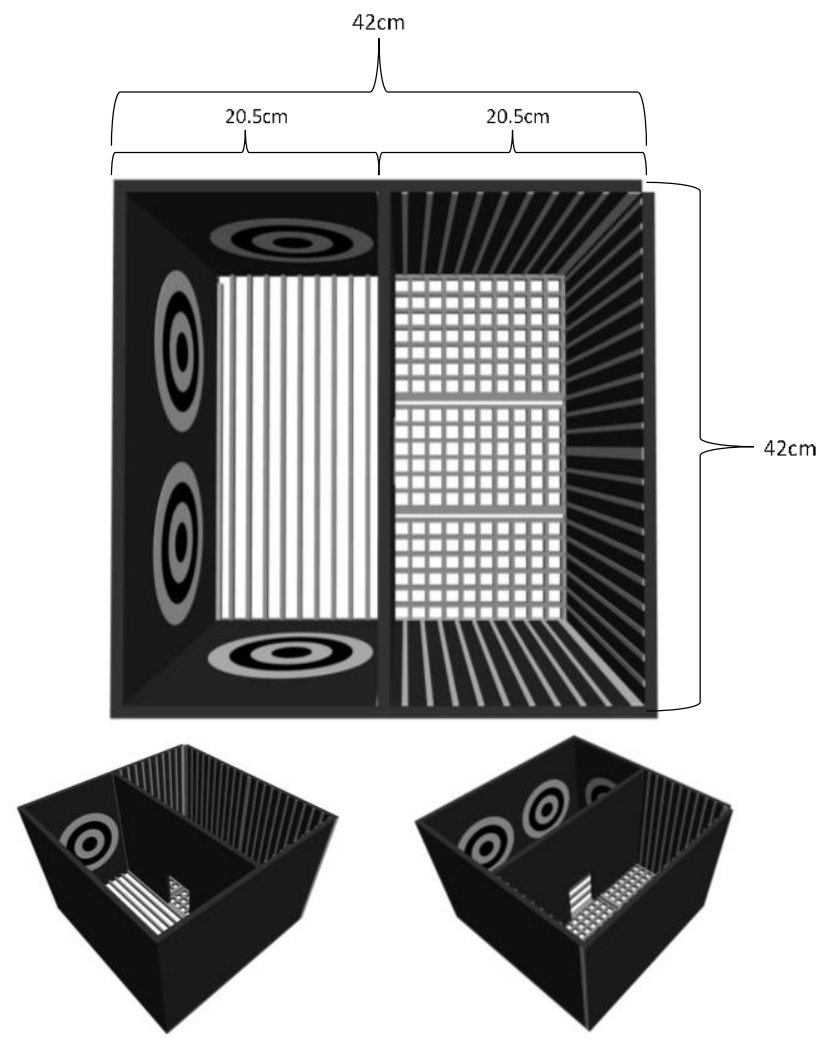




\section{Experimental setting}

The temperature-controlled $\left(21^{\circ} \mathrm{C} \pm 2\right)$ room in which the experiment took place included locomotor chambers around the exterior in closable cabinets and a table in the centre. A red light was used to illuminate the room due to the animal's inability to perceive red light, while maintaining the animals' active period. A white noise generator was used to mask external sound as well as mask the vocalisations from animals in other chambers. Animals were transported into the experimental room in their home cages and placed on the centre table and allowed to habituate for 15-20 minutes prior to the experiment commencing.

Procedure

Prior to the preconditioning phase, pairs of animals were selected based on their genotype and weight, whereby animals were only paired with other animals of the same genotype to avoid genotypical behaviours being masked, as well as avoid dominant behaviour occurring between significantly different sized animals.

Day 1: Preconditioning phase. Following the habitation period, animals were placed in the centre of the apparatus and allowed to freely explore the two chambers to establish any pre-existing bias which may occur due to environmental cues (e.g. tactile differences between the grid and bar flooring). The animal's locomotor activity was tracked using Med associates software to determine the time spent in each chamber throughout the 10 minute session. All animals, irrespective of genotype showed some preference for the chamber with grid flooring, thus following the biased approached, this chamber was used for the later unpaired conditioning phases.

Days 2-11: Conditioning phases. The conditioning phases ran for ten consecutive days with two sessions run per day (one paired and one unpaired). Sessions lasted 10 minutes and were run during the first and last 3 hours of the dark cycle and to ensure the greatest amount of interaction while maintaining the greatest interval between conditioning sessions. The two conditioning 
phases (paired and unpaired) were alternated between morning and night, resulting in a pseudocounterbalanced design. During these phases, a blockage was in place to keep the subject/s in the desired chamber for the remained of the session.

Paired conditioning phases. During the paired conditioning phases, pairs of animals were placed into the centre of the bar floored chamber and allowed to interact for the entire 10minute session. Each session was recorded using an infrared camera located directly above the chamber for later analysis of play behaviours. Following the completion of this session, the animals were removed and placed back into their isolated home cages and returned to the housing room. Chambers were cleaned between sessions using $70 \%$ ethanol to eliminate any residual scents while may affect preference.

Unpaired conditioning phases. During the unpaired conditioning phases, animals were individually placed into the grid floored chamber for the 10-minute session. All procedures were identical to the paired conditioning phases, with the exception of each animal being run individually in grid floored chamber.

Day 12: Post-conditioning phase. To determine the development of preference, the same procedure as the pre-conditioning phase was used. Animals were individually placed in the apparatus and allowed to freely move between the two chambers. Time spent in each chamber was recorded by Med associates software.

\section{Results}

A one-way ANOVA was used to determine whether any preconditioning differences in preference between genotypes existed., revealing no significant between groups differences were present prior to conditioning $[F(2,29)=1.70, p=.200]$.

Following the statistical method of Douglas et al. (2004), preference coefficients were produced for each genotype; Total time spent in the unpaired chamber is deducted from time 
spent in paired chamber, this value is divided by the total trial duration then multiplied by 100 . A one-way ANOVA found a significant genotype effect $[F(2,29)=4.773, p=.016]$ (figure 2). A post-hoc Tukey analysis was conducted to determine the source of the detected differences, revealing a significant difference between $\mathrm{SERT}^{+/+}$and $\mathrm{SERT}^{-/}$groups $(p=.013)$, indicating a greater preference development for $\mathrm{SERT}^{+/+}$genotype (see figure 2). No significant differences were found between SERT ${ }^{+/+}$and SERT ${ }^{+/-}(p=.151)$, nor between the SERT ${ }^{+/-}$and $\mathrm{SERT}^{-/-}$ genotypes $(p=.416)$.

Furthermore, to determine which groups developed a preference, one-sample t-tests were performed to determine whether preference coefficients for each genotype significantly differed from zero. The preference coefficients for the SERT ${ }^{+/+}$group differed significantly from zero $[t$ $(9)=2.62, p=.028]$ indicating a preference for the paired chamber. For the SERT ${ }^{+/-}$group, preferences coefficients did not differ significantly from zero $[t(11)=-.05, p=.961]$, suggesting no development of preference. Finally, the $\mathrm{SERT}^{-/-}$group showed a negative shift in preference which approached significance $[t(9)=-1.99, p=.078]$, indicating that the $\mathrm{SERT}^{-/ \text {group }}$ trended towards an aversion for the paired chamber. 
Figure 2.

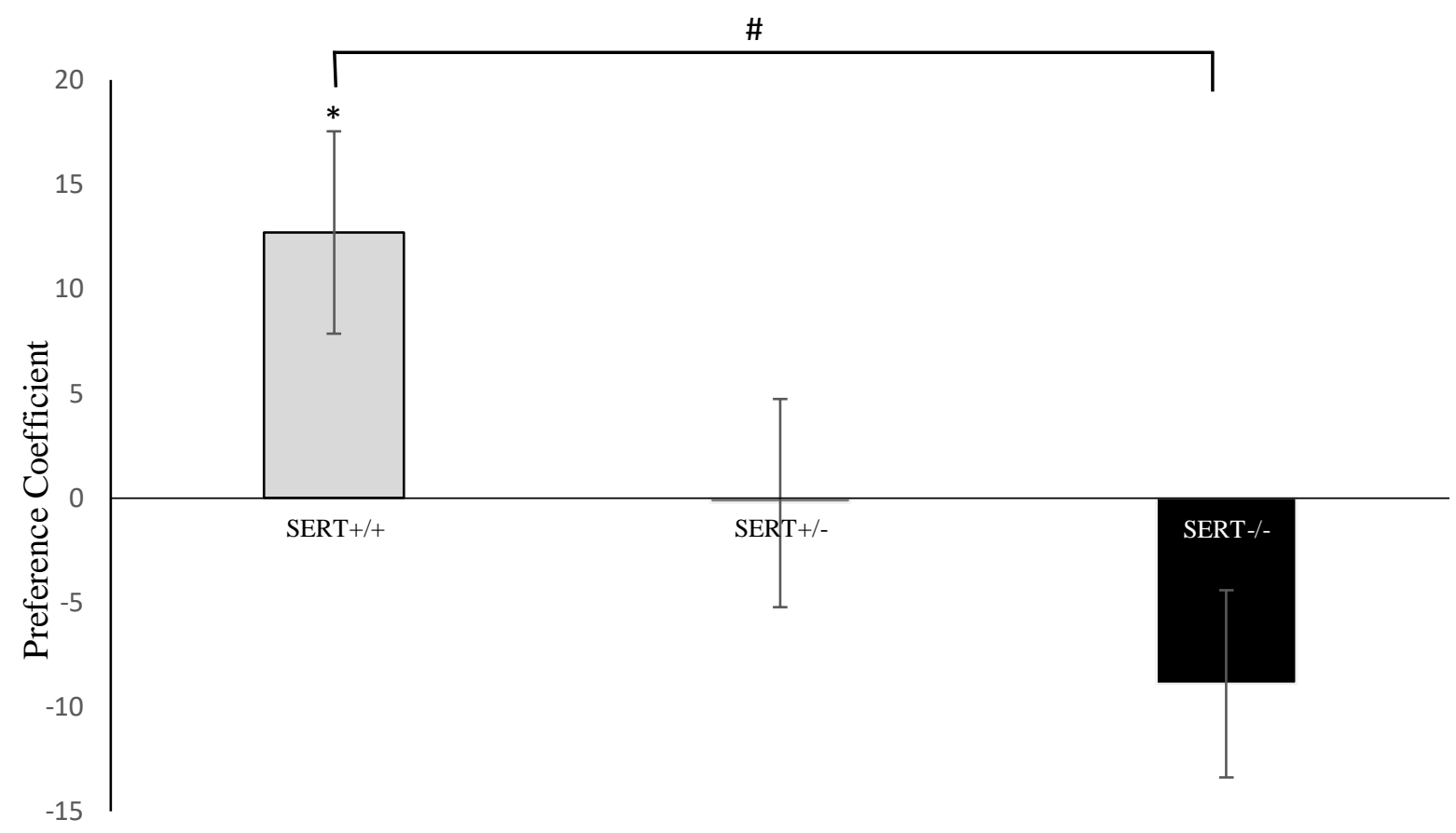

Figure 2. Mean preference coefficients $\left(\frac{\text { Time in paired -Time in unpaired }}{\text { total time } \times 100}\right)$ from the CPP experiment, indicative of the development of preference for the chamber containing the stimulus. Positive values indicate a preference for the paired chamber, while negative values indicate an aversion. Error bars represent the standard error of the mean ( \pm SEM)

$*$ Indicates significant preference development $(p<.05)$

\# Indicates significant between group differences $(p<.05)$

\section{Experiment 1b. Follow up analysis of play behaviour}

To determine whether the preference shift was due to a reduction in reward sensitivity or in social interaction per se video recordings were made. These videos were coded to measure social and non-social behaviour. Following to behavioural criteria of J. R. Homberg et al. (2007), eight behaviours were operationally defined to maintain consistency; non-social behaviour and social behaviour, of which social behaviours were further refined into pinning, pouncing, boxing, and chasing, while non play social interaction included social grooming/exploration, and non-playful indirect social interaction. 
Due to the rewarding nature of play behaviour, we hypothesised that play behaviour would be positively correlated with preference coefficients. Furthermore, based on Homberg and colleagues (2007), we predicted a reduction in play behaviour in the SERT ${ }^{-/}$group compared to the $\mathrm{SERT}^{+/+}$group, with SERT ${ }^{+/-}$showing intermediate levels, in line with the gene-dose effect relationship.

Due to time constraints, means and standard deviations were computed for overall playrelated and non-play related social interaction over the first five days of pairing for 12 of the animals used in the CPP experiment (see Tables $2 \& 3$ ). Pearson's correlations were computed to examine the association between the type of behaviour exhibited during each session and preference coefficients (table 4). Moreover, means and standard deviations for individual behaviours were computed, however, no further analyses were conducted (appendices 1-7) Overall play behaviour, and non-play behaviour were not significantly correlated with preference coefficients.

Table 2.

Means and Standard Deviations for Play Behaviour

\begin{tabular}{c|c|cccccc}
\hline \hline & & Day 1 & Day 2 & Day 3 & Day 4 & Day 5 & Overall \\
\hline SERT & Mean & $54.70 \%$ & $61.44 \%$ & $65.90 \%$ & $69.54 \%$ & $69.72 \%$ & $64.26 \%$ \\
$+/+$ & Standard Deviation & 6.68 & 3.85 & 4.36 & 0.48 & 1.02 & 2.83 \\
\hline \multirow{2}{*}{ SERT } & Mean & $54.58 \%$ & $58.57 \%$ & $71.40 \%$ & $61.88 \%$ & $53.54 \%$ & $59.99 \%$ \\
$+/-$ & Standard Deviation & 2.99 & 1.91 & 3.64 & 2.76 & 4.56 & 3.21 \\
\hline \multirow{2}{*}{ SERT -/- } & Mean & $48.25 \%$ & $55.59 \%$ & $64.47 \%$ & $63.09 \%$ & $67.38 \%$ & $59.75 \%$ \\
& Standard Deviation & 3.97 & 1.36 & 0.99 & 1.88 & 1.21 & 3.47 \\
\hline
\end{tabular}


Table 3 .

Means and Standard Deviations for Non-Play Behaviour

\begin{tabular}{c|c|cccccc}
\hline \hline \multicolumn{2}{c}{ Mean } & $28.61 \%$ & $24.93 \%$ & $25.80 \%$ & $21.78 \%$ & $25.03 \%$ & $25.23 \%$ \\
\hline SERT & Standard Deviation & 3.39 & 2.80 & 3.19 & 0.74 & 1.00 & 1.09 \\
\hline SERT & Mean & $32.83 \%$ & $32.69 \%$ & $25.85 \%$ & $27.54 \%$ & $13.58 \%$ & $26.50 \%$ \\
$+/-$ & Standard Deviation & 1.55 & 1.69 & 1.74 & 3.07 & 2.31 & 3.51 \\
\hline \multirow{2}{*}{ SERT -/- } & Mean & $39.09 \%$ & $34.95 \%$ & $28.95 \%$ & $33.57 \%$ & $30.24 \%$ & $33.36 \%$ \\
& Standard Deviation & 0.87 & 1.62 & 2.53 & 1.93 & 0.98 & 1.80 \\
\hline
\end{tabular}

Table 4.

Correlation Matrix of Social Behaviours and Preference Coefficients

\begin{tabular}{|c|c|c|c|c|c|c|}
\hline & & Day 1 & Day 2 & Day 3 & $\overline{\text { Day } 4}$ & Day 5 \\
\hline \multirow{2}{*}{ Non-Play } & Correlation Coefficient (r) & -.016 & .208 & .083 & .369 & .015 \\
\hline & Significance (p) & .953 & .439 & .760 & .159 & .957 \\
\hline \multirow{2}{*}{ Play } & Correlation Coefficient (r) & -.296 & -.404 & -.109 & $-.513^{*}$ & $\begin{array}{l}.149 \\
-.149\end{array}$ \\
\hline & Significance (p) & .265 & . 121 & .687 & .042 & .582 \\
\hline
\end{tabular}

To examine the effect of reduced serotonin transporter expression on the exhibition of play related social interactions over time, means and standard deviations were computed for the proportion of time engaging in social play behaviour for each day. A mixed ANOVA was conducted with time (Days 1-5) as the repeated measure, and genotype $\left(\mathrm{SERT}^{+/+}, \mathrm{SERT}^{+/}, \mathrm{SERT}^{-}\right.$ $\left.{ }^{\prime}\right)$ as the between groups variable. As determined by Mauchley's test, the assumption of sphericity was not violated, thus no corrections were required. A significant main effect of day was uncovered $[F(4,20)=8.164, p<.001]$, suggesting that irrespective of genotype, the proportional duration of play related social behaviour increased over time (see figure 3). However, no significant genotype effect, or interaction was present. 
Figure 3.

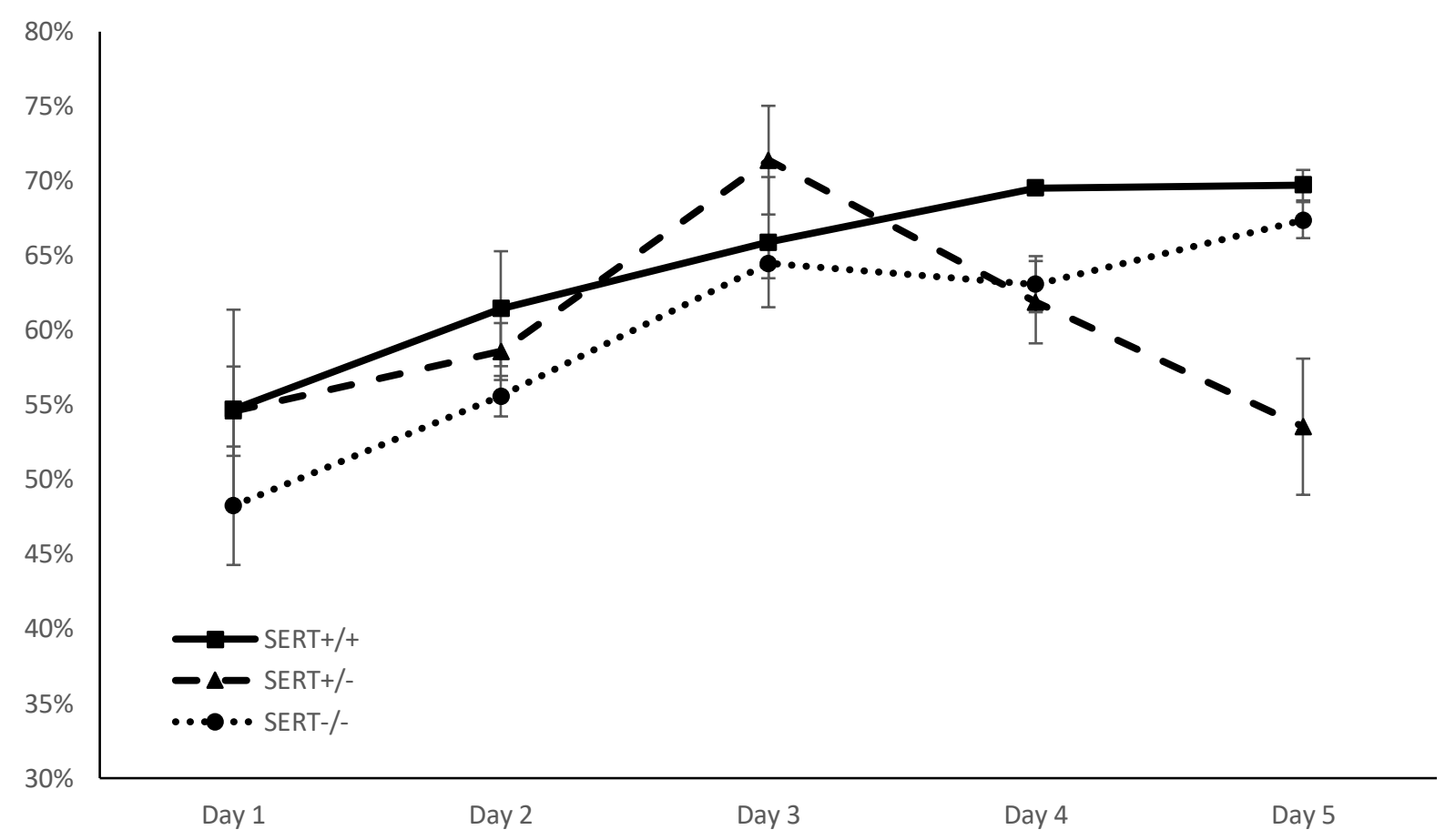

Figure 3. Means and \pm SEM of the proportion of time spent engaging in play related social interaction (total trail duration 600 seconds). Consecutive days are presented on the $x$ axis, while the percentage of time is depicted through the $y$ axis. SERT ${ }^{+/+}$are represented by the solid line, $\mathrm{SERT}^{+/}$are represented by the dashed line, while the dotted line indicates the $\mathrm{SERT}^{-/}$group. A significant main effect of time was discovered $(p<.001)$, no further effects or interactions were uncovered.

A mixed ANOVA was also conducted with time (Days 1-5) as the repeated measure, and genotype $\left(\mathrm{SERT}^{+/+}, \mathrm{SERT}^{+/-}, \mathrm{SERT}^{-/}\right)$as the between groups variable for social non-ply behaviour. The assumption of sphericity was not violated, thus no corrections were used. A significant main effect of time was revealed $[F(4,20)=4.55, p=.009]$ indicating that irrespective of genotype, non-play behaviours reduced as a function of time (see figure 4). Furthermore, a significant main effect of genotype was uncovered $[F(2,5)=6.49, p=.041]$, indicating that there was an effect of genotype, irrespective of day. Follow-up pairwise comparisons were conducted indicating that the SERT $\mathrm{S}^{-/}$ group engaged in significantly greater non-play social behaviour than both the $\mathrm{SERT}^{+/+}$group (p $=.025)$ and the $\mathrm{SERT}^{+/-}$group $(\mathrm{p}=.031)$. Finally, the genotype $\mathrm{x}$ day interaction was not significant. 
Figure 4.

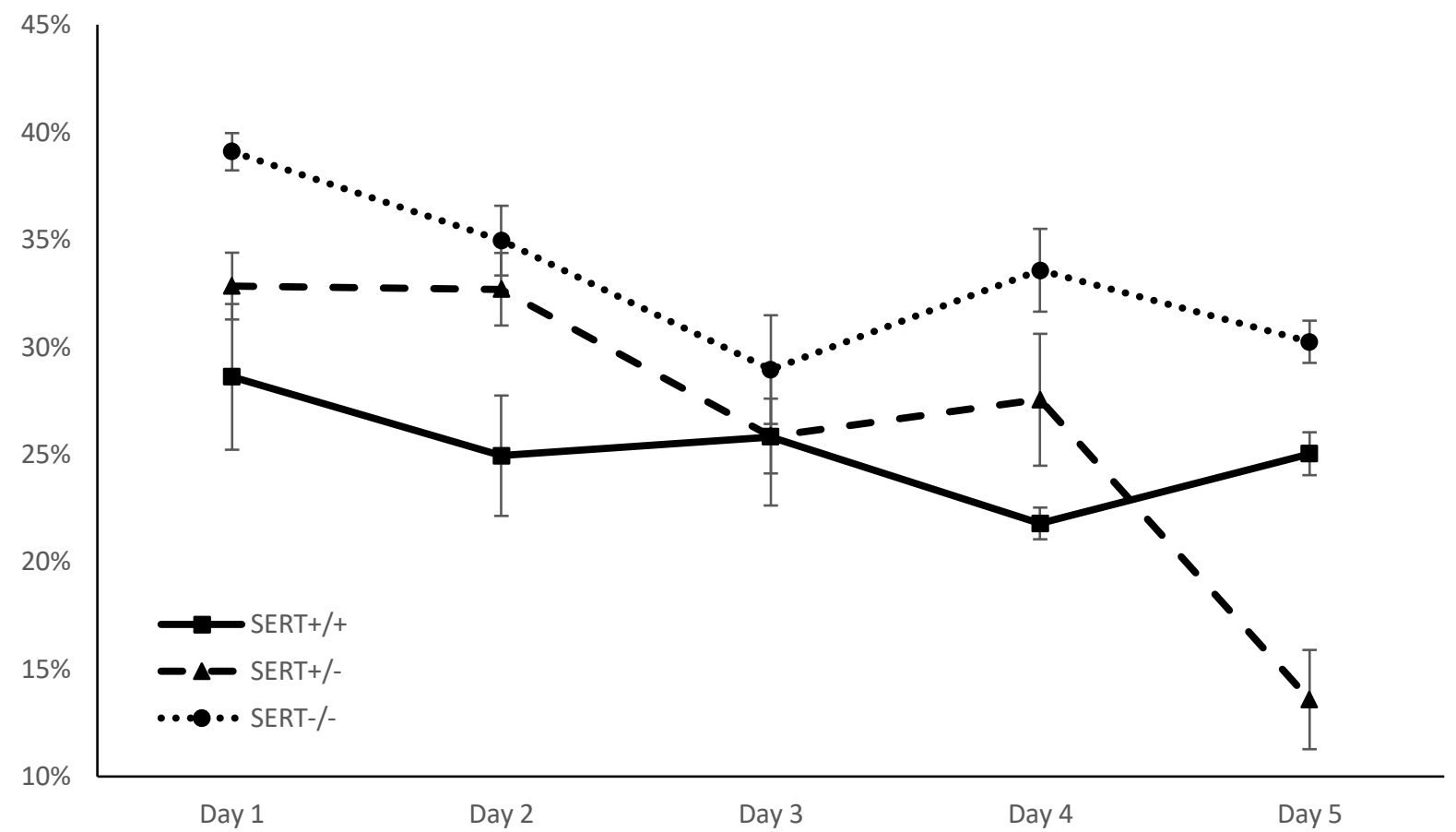

Figure 4. Means and \pm SEM of the proportion of time spent engaging in non-play related social interaction (total trail duration 600 seconds). Consecutive days are presented on the $x$ axis, while the percentage of time is depicted through the $y$ axis. SERT $^{+/+}$are represented by the solid line, SERT ${ }^{+/}$are represented by the dashed line, while the dotted line indicates the $\mathrm{SERT}^{-/}$group. A significant main effect of time was found $(p=.009)$.

Furthermore, a significant main effect of genotype was uncovered $(p=.041)$, whereby the SERT $^{-/}$group engaged in more non-play related interaction than the SERT ${ }^{+/-}(p=.031)$ and SERT $^{+/+}(p=.025)$ groups. No interactions were uncovered.

\section{Discussion}

The present experiment sought to examine the influence of the partial and complete genetic abolition of the serotonin transporter on social reward. To assess this, the CPP paradigm was used, with the level of preference development being indicative of experiencing reward from social stimuli. Based on the comprehensive study by Douglas et al. (2004), detailing the factors which increase the development of social preference, it was hypothesised that adolescent rats with an uncompromised serotoninergic system would develop a significant preference for the location 
where social pairings occurred. Furthermore, based on the human and animal studies examining the influence of a compromised serotonergic system on social behaviour and reward (J. R. Homberg et al., 2007; Rosenthal et al., 1998), it was hypothesised that a partial deletion in serotonin transporter expression $\left(\mathrm{SERT}^{+/}\right)$would lead to a small reduction in preference development, while a complete elimination of the serotonin transporter expression $\left(\mathrm{SERT}^{-/}\right)$would result in a larger reduction, or even an absence of preference development. In line with our hypotheses, the SERT $^{+/+}$animals showed a significantly greater preference for the social compartment than the $\mathrm{SERT}^{-/}$group, and while not reaching significance, the $\mathrm{SERT}^{+/}$group fell midway between the two extremes, thus demonstrating a gene-dose response-like effect. Further analyses revealed the development of a significant preference was limited to the SERT ${ }^{+/+}$group (see figure 2). In fact, while the SERT ${ }^{+/}$group showed no preference, the SERT ${ }^{-/}$group even showed a (nonsignificant) tendency towards an aversion ( $p=0.08$, see figure 2). These results indicate that a partial reduction in serotonin transporter expression results in an absence of social reward, while a complete reduction may result in social stimuli being aversive in nature.

Aligning with prior research and our understanding of serotonin's involvement in social behaviour, it has been demonstrated that increased extracellular serotonin from gestation results in a diminished preference for novel social interaction (Moy et al., 2009). However, the precise mechanisms underlying this effect have yet to be fully established. To further elucidate the underlying mechanisms behind the development of preference, or lack thereof, social behaviour was coded as either play related, or non-play related social behaviour in accordance with $\mathrm{J}$. R. Homberg et al. (2007). As Homberg and colleagues demonstrated a reduction in social play behaviour in $\mathrm{SERT}^{-/}$animals, while non-play related social behaviour remained unaffected, it was hypothesised that these results would be replicated with the SERT ${ }^{+/-}$falling midway between the extremes, producing a gene-dose response. 
These hypotheses were not supported by the data, as unlike Homberg and colleagues, we did not find any significant genotype differences in play related behaviours. There are several possible explanations for these discrepancies. First of all, it is known that play behaviour strongly depends on external factors, such as lightning, size of the experimental chamber, period of previous social isolation etc (Vanderschuren, Niesink, Spruijt, \& Van Ree, 1995). Secondly, we focused on total duration, while Homberg et al only measured frequency of behaviours. Thirdly, the duration of each trial differed, this experiment used 10 minute trials as it has been previously shown that play behaviours decline after 10 minutes of interaction (Douglas et al., 2004), while Homberg et al used a 15 minute trial duration. Finally, we measure repeatedly over a period of 5 days, while Homberg et al only assessed play behaviour in a single experiment.

Intriguingly, neither play, nor non-play related social behavioural was associated with preference coefficients when examining all animals. This may in part be due to the relatively small sample size (time constraints did not allow us to analyse all videos from all animals). However, it is also possible that social reward (as reflected in the preference coefficients) is fundamentally different from social (play) behaviour per se. The finding that SERT-/- rats had significantly reduced social reward, but showed no difference in play behaviour and even an increase in nonplay social behaviour supports this view.

A possible mechanism by which the trend towards aversion in the $\mathrm{SERT}^{-/-}$occurred may be the experience of stress from being placed in the environment which was deemed to be less favourable in the pre-conditioning test. All animals showed an initial preference for the chamber which contained the grid flooring, which is likely due to more stable footing as the bar floor contained wide gaps between bars. Being place in a chamber which may cause discomfort would induce a stress response While this stress response would occur in uncompromised animals, this is likely offset by the experience of reward from social interaction (Ulrich-Lai et al., 2010). However, the compromised reward system of the SERT $T^{-/}$animals may have failed to offset the 
effect of stress, resulting in a measure of environmental stress, possibly due to increased neuronal activity in the amygdala (Hariri et al., 2002; Krishnan \& Nestler, 2008; Montañez, Munn, Owens, Horton, \& Daws, 2014).

It should be noted that due to the extended duration of isolation prior to the experiment commencing, stress may have significantly impacted the observed results. Social isolation has been shown to be a significant stressor to rats due to their highly sociable nature resulting is significant HPA axis dysfunction (Serra, Pisu, Floris, \& Biggio, 2005), and increased depressive and anxietylike behaviours (Brenes, Rodríguez, \& Fornaguera, 2008; Weiss, Pryce, Jongen-Rêlo, Nanz-Bahr, \& Feldon, 2004). Furthermore, the negative impact of social isolation has been shown to be exacerbated between PND25-45, as this is a critical time of social development (Einon \& Morgan, 1977). While stress is likely to have impacted all genotypes, the experience of stress may have been exacerbated by the genetic reduction of serotonin transporter expression, thus comprising a geneenvironment interaction. This interaction has been demonstrated in rhesus macaques exhibiting similar serotonergic dysregulation to the human 5-HTTLPR s-allele carriers and SERT knock out rats, whereby HPA axis activation in response to stressors were shown to amplified in s-allele carriers (Barr et al., 2004). Similarly, Carola et al. (2008) demonstrated that poor maternal care significantly impacted anxiety like behaviours in $\mathrm{SERT}^{+/-}$but not $\mathrm{SERT}^{+/+}$mice, indicating the presence of the gene $x$ environment interaction in SERT knockout rodents.

The impact of stress during early development aligns with studies on humans, whereby exposure to adverse experiences such and abuse and neglect or other significant stressors during childhood and adolescents have been shown to predict negative outcomes psychiatric illness (Hazel, Hammen, Brennan, \& Najman, 2008). Furthermore, the gene x environment interaction between 5-HTTLPR polymorphism and early stress has been well documented in humans, being associated with depression, and anxiety, among other psychiatric disorders (Caspi \& Moffitt, 2006; Taylor et al., 2006), whereby s-allele carriers reporting a higher number of stressful events, or 
chronic stressors were found to be at a significantly greater risk of developing depression (Caspi et al., 2003) thus likely benefiting the translatability of these results to the human condition.

Previous studies have demonstrated impaired social communication as a result of alterations in serotonin homeostasis (Ellenbogen et al., 1996). These studies generally focus on the reduction of serotonin through depleting the precursor tryptophan, thus resulting in the inability to replenish serotonin levels. As a result of this reduction, mood and social deficits are mostly reported after tryptophan depleting in adults. These deficits return to baseline as serotonin levels normalize, while existing deficits are often alleviated through chronic increases in serotonin (Locher et al., 2017). However, through the genetic reduction in serotonin transporter expression, as seen in $\mathrm{SERT}^{+/}$and $\mathrm{SERT}^{-/}$rats, extracellular serotonin levels are increased, yet as demonstrated in this study produce depressive-like behaviours such as social anhedonia. This dissociation is referred to as the serotonin paradox, and while the precise mechanisms remain to be fully elucidated, some possible mechanisms have been proposed. Given then serotonin paradox implicates increased extracellular serotonin throughout development as underlying the depressive characteristics produces, the most promising theory posits that early increasing in serotonin induce multiple developmental effects, as well as the downregulation of post-synaptic receptors, resulting in a reduced receptor activation (Fox, Stein, French, \& Murphy, 2010). This downregulation of serotonin receptors likely underlie the social deficits in $\mathrm{SERT}^{+/-}$and $\mathrm{SERT}^{-/}$rats, as demonstrated by Dunn, Corbett, and Fielding (1989), administration of selective 5-HT ${ }^{1 \mathrm{~A}}$ agonists; buspirone, fepirone, and 8-hydroxy-2-(di-n-propylamino) tetralin (8-OH-DPAT) all produced significant increases in social interaction. Furthermore, using CPP, intraperitoneal administration and direct microinjections into the raphe-nuclei of 8-OH-DPAT were sufficient in producing a significant preference, thus implicating $5-\mathrm{HT}^{1 \mathrm{~A}}$ agonism in social interaction and reward.

Most importantly, the data of the present study provide further validity for the SERT ${ }^{-/-}$ rats as a model for depression. While multiple studies have found a reduction in social interaction 
in depressed patients (Lewinsohn, Mischel, Chaplin, \& Barton, 1980), these self-reported data may be skewed by the negative biases associated with depression (Pyszczynski, Hamilton, Herring, \& Greenberg, 1989), while the frequency of interaction may be underreported due to feelings of isolation and reduced emotional valence during socialisation (Cacioppo, Hawkley, \& Thisted, 2010). One study addressed these methodological issues used diary reports whereby interactions were reported in detail, including the relationship with the person, gender, and personal experience of the interaction (Nezlek, Imbrie, \& Shean, 1994). Across all measures of the quantity of interactions, including total number of interactions, duration of interaction, and proportion of time spent interacting per day, no significant differences were found between the depressed and nondepressed sample. However, enjoyment, intimacy, responsiveness, influence, and confidence measures for each interaction showed significant reductions in depressed participants. These results are very similar to the findings reported here, while the overall duration of play behaviour was not reduced (and in fact the duration of social non-play behaviour was even slightly increased in $\mathrm{SERT}^{-/-}$rats), the rewarding effect of interactions is significantly reduced in rats with a genetically compromised SERT. These findings suggest that the $\mathrm{SERT}^{-/-}$rats represent a valid model for the social anhedonia observed in patients with MDD. 


\title{
CHAPTER THREE
}

\author{
Experiment Two
}

Repeated Testing of Anxiety Using the Modified Successive

Alleys Test 
Patients with major depressive disorders often show comorbid symptoms of anxiety disorders. According to the DSM-V, anxiety disorders are by persistent or irregular panic, fear, and uneasiness, sleep disturbances, agitation, perspiration, shortness of breath, and increased heart rate in the absence of physical exertion (Association, 2013). Comorbidity rates of these disorders are some of the highest in the mental health area with studies suggesting between $50-60 \%$ of people experiencing MDD will also suffer from an anxiety disorder (Fava et al., 2000; Kaufman \& Charney, 2000; Kessler, Nelson, McGonagle, \& Liu, 1996). This is unsurprising given that predominant theories posit that both depressive and anxiety disorders are in essence stress induced disorders (Smoller, 2016), with some suggesting that anxiety generally pre-empts the development of depression (Cole, Peeke, Martin, Truglio, \& Seroczynski, 1998). To further examine this temporal association, Moffitt et al. (2007) analysed the cumulative and sequential comorbidity of depression and generalised anxiety disorder (GAD) using data obtained from the ground breaking Dunedin Multidisciplinary Health and Development Study (Poulton, Moffitt, \& Silva, 2015; Stanton, 1996), where a cohort of 1037 babies born between 1972 and 1973 has been (and continues to be) followed throughout life. The results of this study indicate that while $48 \%$ of lifetime cases of depression experienced anxiety, $72 \%$ of those who experienced GAD also experienced depression within the time course of this study. Further analyses were conducted to examine the potential of a directional relationship which found that while $37 \%$ of cases of depression occurred concurrently or following the development of anxiety, the opposing direction was observed in $32 \%$ of cases of anxiety. These results further support the idea that the association between these disorders are strongly related but further suggests that the relation is more complex than the simplistic directional relationship that has been suggested.

The comorbidity between depression and anxiety disorders is strong enough to prompt the inclusion of the diagnostic criteria of mixed anxiety and depressive disorder in the World Heath Organisations International Statistical Classification of Diseases and Related Health Problems (ICD) (Lam et al., 2012). This combination of disorders has not been without criticism (Goldberg, 
2014), as in recent years there has been a drive towards separating disorders into their individual symptoms to circumvent their heterogeneity (Cuthbert, 2014). Despite the issues with this combination approach, it is important to note that both depressive and anxiety disorders are most commonly treated with selective serotonin reuptake inhibitors (SSRIs) such as fluoxetine, escitalopram, and sertraline (Panel, 1993; Roy-Byrne, Wingerson, Cowley, \& Dager, 1993). Response to these pharmacological treatments are variable, with many patients being prescribed multiple different SSRIs prior to any reduction in symptoms occurring (Berman, Narasimhan, \& Charney, 1997; O'reardon, Brunswick, \& Amsterdam, 2000). Despite the variability in response, SSRIs are the preferred treatment for anxiety disorders as other anxiolytics such as benzodiazepines can impair normal functioning and have a high abuse potential (Longo \& Johnson, 2000). Moreover, the effects of SSRIs in both MDD and anxiety disorders points to a common role for serotonin in these psychiatric disorders.

In order to examine anxious behaviours in animals, a range of behavioural assays have been developed, virtually all are based around the conflict theory, whereby an animal must choose between safety, and a competing (natural) impulse, such as exploration, food etc (Wall \& Messier, 2001) (Cryan \& Sweeney, 2011). J. Olivier et al. (2008) conducted a study examining anxiety like behaviours in SERT knockout rats across the open field, elevated plus maze, home cage emergence paradigms (all based on the conflict between safety and the drive to explore) and novelty suppressed feeding (based on the conflict between safety and the drive to eat). The results showed $\mathrm{SERT}^{-/-}$animals tend to show more anxious behaviours across all paradigms, except for the elevated plus maze which failed to reach significance. While robust, these paradigms may lack the sensitivity to differentiate between high anxiety compared to moderate anxiety, as with the elevated plus maze due to their binary nature. For example, most of the results focus on whether the animal is in location $x$ (low anxiety/safety) or location $y$ (high anxiety/danger) which while useful, remains a crude measure attempting to match a more complex disorder on a spectrum of severity. Moreover, from an ethological point of view, spending more time in a safe environment may be 
beneficial. Indeed, it is difficult to determine in these models where natural cautiousness ends and pathological anxiety starts.

Finally one of the major issues in the present tests of anxiety is the one-trial-tolerance (OT'T) problem, which was first demonstrated by File, Mabbutt, and Hitchcott (1990), whereby prior exposure to the elevated plus maze diminished the effects of anxiolytics, creating issues in testing within subject effects. Furthermore, anxious behaviour diminished across trails resulting in a failure to find significant group effects with only one additional trial (Cook, Crounse, \& Flaherty, 2002; File, Lippa, Beer, \& Lippa, 2004; Hånell \& Marklund, 2014; Lister, 1987). Although Schwarting and Pawlak (2004) showed that a 120 day separation between trials eliminated the emergence of OTT, and Schneider, Ho, Spanagel, and Pawlak (2011) further demonstrated that a novel experimental room with a 28 day interval was sufficient, it is clear that the current generation of anxiety models are not able to measure stable trait-like anxiety states.

The successive alleys test (SAT) was designed to provide a solution for the binary issue mentioned above (Deacon, 2013). Like the elevated plus maze, the SAT contains four segments or "alleys", however, they are in one straight line and, most importantly, have progressively smaller walls and narrower alleys (see figure 1). However, in its original form, the test does not solve the issue of repeated testing. Indeed, unpublished studies from our own laboratory showed that, as with the elevated plus maze, repeated testing in the SAT leads to a reduction in time spent in the more anxiogenic alleys ( 3 and 4 ) and more time spent in the safer alleys (1 and 2). Therefore, we set out to adapt the SAT in an attempt to allow meaningful repeated measures of anxiety. This would not only allow us to more accurately assess trait anxiety but also would make the test suitable for investigating novel treatments.

One core element in the diagnosis of anxiety disorders, is that the symptoms must impact general functioning. While this is somewhat observed in the reduction of exploration, it is better modelled in the novelty suppressed feeding, whereby animals are food deprived, thus the 
acquisition of food or lack thereof is a behaviour essential for survival. We therefore decided to combine the SAT with food rewards in each alley. This, we reasoned, has the added benefit that the baiting of each alley entices exploration of the more anxiogenic alleys, even upon repeated exposure. As a result, several different variables can be measured from the test, including latency to leave alley one, latency to reach alley four, duration in all four alleys, food retrieval behaviour, and ultrasonic vocalizations.

A significant criticism of our current tests of anxiety is related to inferring the emotional state of an animal from its behaviour (Ramos, 2008). Therefore, it is important to incorporate an element which is considered to be a marker of emotional state in the test animal. Very few methods of assessing pure emotional state in rats exist, however, ultrasonic vocalisations (USVs) and facial expressions (Finlayson, Lampe, Hintze, Würbel, \& Melotti, 2016) have been proposed as markers of internal emotional state. Facial expression has been used to detect both positive and negative valence in response to rewarding or aversive stimuli, however, the difficulty of detection makes the assessment of facial expression incompatible with many experimental designs. USVs, on the other hand, represent a method of communication between rats and are relatively easy to record. As a result, it can be incorporated in many experimental set-ups. USVs are generally categorized into two ranges in adult rats, $22-\mathrm{khz}$ (true range: $18-32 \mathrm{khz}$ ) calls which are indicative of a negative emotional state (Jelen, Soltysik, \& Zagrodzka, 2003), while 50-khz (true range 32-96khz) are suggested to indicate a positive emotional state, furthermore, individual rats tend to only fluctuate by around 1-6khz of their average emitted frequency in these ranges. To support this claim, Thompson, Leonard, and Brudzynski (2006) administered amphetamine, a highly rewarding drug due to its action on the dopamine system, to rats and recorded the USVs emitted. A dose response increase of the number of 50-khz calls in response to amphetamine administration was observed. These results were further supported by Burgdorf, Panksepp, and Moskal (2011) who observed 50-khz call increases in response to enhanced dopaminergic activity, whilst further suggesting the involvement of the cholinergic system, which is often involved in number of defensive behaviours, 
to underlie the increase in $22-\mathrm{khz}$ calls in response to negative stimuli. The authors also suggest that these two types of calls never occur at the same time and due to their association with associated behaviour and stimuli are clear measures of negative or positive emotional experiences. Finally, USV data was obtained alongside facial expressions by Finlayson et al. (2016), who found a significant correlation between these measures. Finally, since latency and duration are heavily reliant on exploratory behaviour, the inclusion of food retrieval and particularly USV allows for the assessment of motor behaviour-independent aspects of anxiety.

Given these association, it is important that measures of anxiety should include USVs to further validate what is being inferred from the observed behaviour of rats. Through the modified SAT and the inclusion of USVs, we propose a more detailed measure of anxiety both from a behavioural and emotional perspective, allowing the development of a behavioural trait as opposed to a single behavioural state score. Furthermore, given the number of studies examining the SERT knockout mutation on anxiety behaviours, this mutation is a strong candidate for examining anxious behaviour in a novel paradigm.

The present study aimed to establish a behavioural test of anxiety which circumvents the core issues present in more frequently used assessment of anxiety in animal models. Through the use of the modified SAT (mSAT), we hope to provide a measure that does not rely on a dichotomous design, thus likely being more sensitive to differentiating between severities of anxiety-like behaviours. Furthermore, the inclusion of baited alleys will be incorporated to circumvent the OTT issue previously discussed.

To determine whether the mSAT overcomes the OTT issue, multiple trials were conducted over the course of a week. It was hypothesised that there might be a significant effect of day, however this would be independent of genotype, which would indicate that the mSAT is resistant to the OTT issue. Furthermore, to ensure the construct (anxiety) being assessed during each trial did not change, Cronbach's alpha was computed for each behavioural measure. We hypothesised 
that a sufficient alpha value (greater than .7) would be obtained, suggesting that the anxiety remained stable over all trials. Furthermore, to further validate anxiety we hypothesised that the proportion of $50 \mathrm{khz}$ USVs (indicative of a positive emotional state) would be inversely correlated with latency to enter alley four, while being positively associated with the duration spent in alley four (as these latter two are measures of a negative emotional state). Given the results from the previous chapter, that $\mathrm{SERT}^{-/-}$rats show more depression-like social anhedonia and the strong comorbidity between depression and anxiety, we hypothesise that $\mathrm{SERT}^{-/}$rats show the most anxious and the SERT $+/+$ rats the least anxious phenotype. This would also be in line with previous data from more classical anxiety tests such as the elevated plus maze, home cage emergence and one field test (J. Olivier et al., 2008). Thus, we predict that SERT ${ }^{-/-}$rats show the shortest time spent in, and the longest latency to enter alley 4. Likewise, we predict that the SERT ${ }^{-}$

${ }^{1-}$ had the lowest food retrieval scores (as explained below, lower retrieval scores are indicative of a more anxious phenotype). In addition, we expect these genotype differences to remain stable over time, although the overall anxiety levels may improve with repeated exposure to the mSAT.

\section{Method}

Subjects

A separate cohort of animals was used for this experiment consisting of 27 male Wistar rats. Three different genotypes were used; 6 homozygous SERT knockout $\left(\mathrm{SERT}^{-/}\right), 11$ heterozygous SERT knockout $\left(\mathrm{SERT}^{+/}\right.$), and 10 Wildtype animals lacking the SERT knockout mutation $\left(\mathrm{SERT}^{+/+}\right)$. All subjects were of adult age ranging between postnatal days (PND) 71-100. Animals were maintained at $85-90 \%$ of their free feeding weight for at least one week prior to the start of testing to establish food directed motivation (Woods \& Begg, 2015). To maintain this weight, 12-15g of food was provided daily in the evening. On testing days, animals were fed at a random time point following testing, with at least a one hour variable delay between the end of their test session and being fed to maintain food motivation (Boulos, Rosenwasser, \& Terman, 
1980). All rats were housed in polycarbonate cages (2-3 per cage) in a temperature and humiditycontrolled environment, $21^{\circ} \mathrm{C} \pm 2$ and $55-60 \%$ respectively. Animals were housed on a reverse light cycle (lights on at 1900-0700 hours) to ensure testing occurred during active periods. Animals were bred and housed in the Victoria University of Wellington vivarium in accordance with the Animal Welfare Act 1999, with all procedures being approved by the VUW Animal Ethics committee (AEC number 24384).

Experimental setting

The experimental room was temperature controlled $\left(21^{\circ} \mathrm{C} \pm 2\right)$ consisting of the SAT apparatus set up in the centre of the room with a high definition recorder set up above the apparatus, providing a bird's eye view to monitor the animal behaviour. Dim LED lighting was used and maintained at approximately, 5, 8, 9, and 10 lux (alleys 1 through 4 respectively), the minimum threshold at which detection through the camera was possible. Furthermore, a 30x30 cm novel environment chamber containing an UltraMic microphone (250K 16 bi; Java Sound) was located in the corner of the room, outside of direct view of the SAT apparatus.

\section{Apparatus}

The successive alleys apparatus was constructed using gloss black polycaronate pannels, joined with epoxy resin. Each alley slots together to create a complete unit which can be broken down for thourough cleaning. Alleys progressively reduce in runway width and wall height to increase the induction of anxiety. Each alley is $45 \mathrm{~cm}$ in length $(180 \mathrm{~cm}$ total), while alley one is $29 \mathrm{~cm}$ in height and $9 \mathrm{~cm}$ in width, alley two is $6 \mathrm{~cm}$ by $8 \mathrm{~cm}$, alley three is $2.5 \mathrm{~cm}$ by $6.5 \mathrm{~cm}$, and alley four is $1 \mathrm{~cm}$ by $3.5 \mathrm{~cm}$ (table 1 \& figure 1$)$. 
Figure 1.
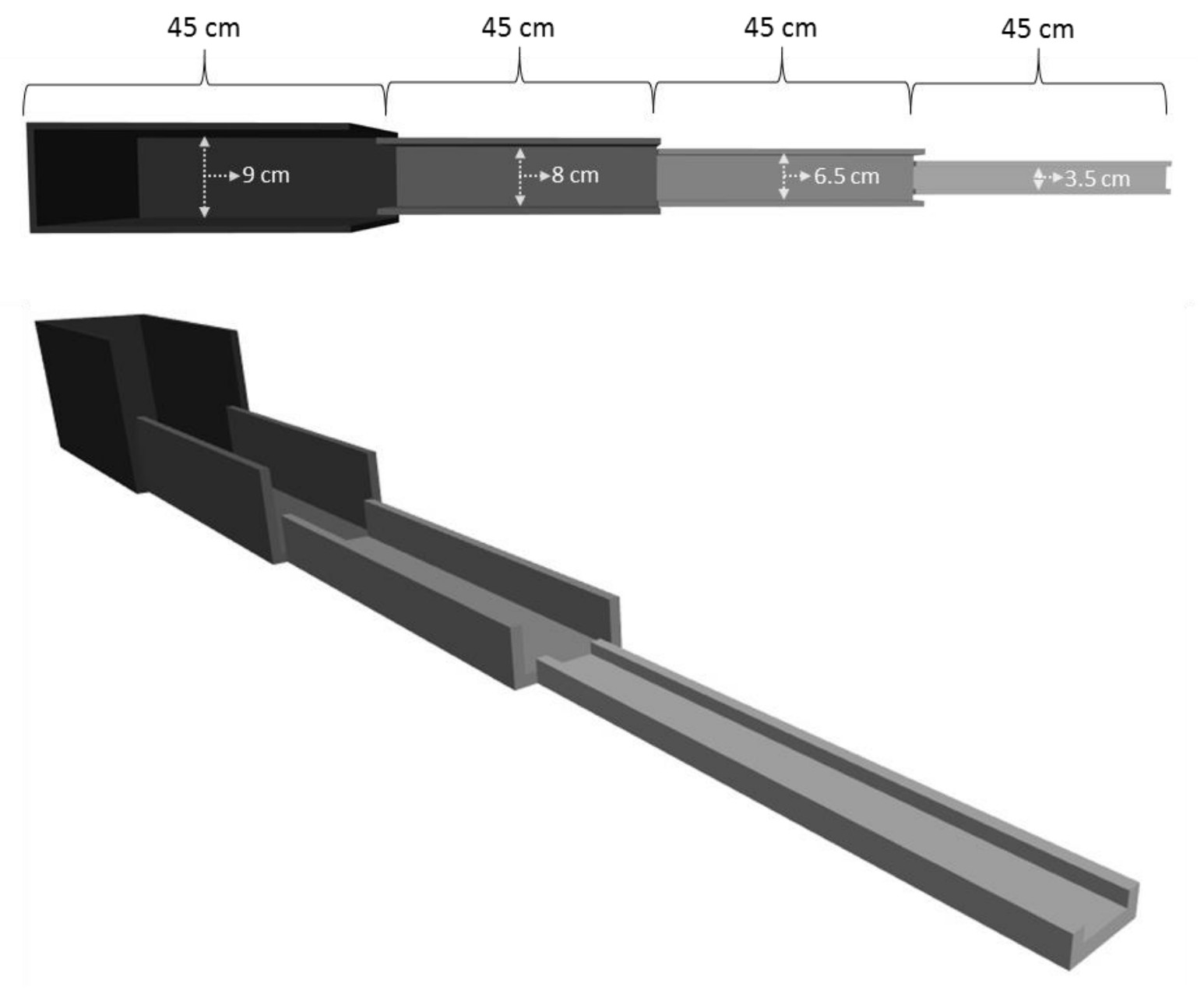

Table 1.

Dimensions of the Successive Alleys Apparatus

\begin{tabular}{|c|c|c|c|c|}
\hline Alley & Length & Width & Wall height & $\begin{array}{c}\text { Hypothesised Anxiogenic } \\
\text { Effect }\end{array}$ \\
\hline 1 & $45 \mathrm{~cm}$ & $9 \mathrm{~cm}$ & $29 \mathrm{~cm}$ & Low \\
\hline 2 & $45 \mathrm{~cm}$ & $8 \mathrm{~cm}$ & $6 \mathrm{~cm}$ & Modest \\
\hline 3 & $45 \mathrm{~cm}$ & $6.5 \mathrm{~cm}$ & $2.5 \mathrm{~cm}$ & Moderate \\
\hline 4 & $45 \mathrm{~cm}$ & $3.5 \mathrm{~cm}$ & $1 \mathrm{~cm}$ & High \\
\hline
\end{tabular}

\section{Procedure}

Following the pilot procedure, testing occurred over the duration of eight days; an initial four days of testing, followed by a two day break, then two additional days of testing. On testing 
days, animals were transported from the housing room into the testing room in their home cages and allowed to habituate for at least 30 minutes prior to any testing.

Animals were placed into a novel experimental box five minutes prior to being placed in the SAT alleys apparatus to record anticipatory USVs. USV data were collected using an UltraMic (205K, 16 bi; Java Sound) and analysed using Raven software, whereby all calls present in each 600 second file were manually selected to ensure a high degree of accuracy. Calls falling with in the 18$32 \mathrm{khz}$ range were coded as $22 \mathrm{khz}$ calls, while $50 \mathrm{khz}$ calls were those between $32-72 \mathrm{khz}$ (Jelen et al., 2003). A large degree of variation was detected between animals in respect to the total number of calls made, to correct for this variation, the number of $50 \mathrm{khz}$ call was divided by the total number of calls within the $18-72 \mathrm{khz}$ range, producing a $50 \mathrm{khz}$ percentage which would indicate the interplay between high and low frequency calls. Increased high frequency percentage would be indicative of both increased $50 \mathrm{khz}$, and reduced $22 \mathrm{khz}$ calls, and, as such reflect a more positive emotional state. Following the completion of the USV recordings, the animal was removed from the novel environment box and placed facing the back of alley one of the successive alleys. Locomotor activity was recorded using a camera positioned directly above the apparatus and using Ethovision software to track movement. Total duration in each alley, as well as latency to enter each alley was measured using Ethovision tracking, while consumption of the Frootloops and location of consumption was manually recorded. The SAT was cleaned and baited (half a Frootloop in the centre of each alley) between each session.

Each day of testing, the bait position was changed in correspondence to the behaviour that was observed the prior day. Alleys were baited from the highest anxiety inducing alley where consumption occurred on the previous session and onwards. For example, if an animal consumed the bait from each alley, in the alley they were presented, the following day bait would only be presented in the fourth alley, however, if all bait from each alley was retrieved and consumed in alley two, the following session, alley two, three, and four would be baited. 
To score retrieval behaviour, each alley was allocated with a value whereby bait consumed within that alley would be given either one, two, three, or four points (alleys 1 through 4 respectively) (figure 1). Alleys which induce a greater anxiogenic effect would be attributed a higher score, thus animals scoring higher in retrieval behaviour consume the bait in more anxiogenic alleys, in turn implying a lower level of anxiety. For example, if an animal consumed all bait in the alleys in which they were presented, the animal would be given a score of 10 , while an animal who retrieves the bait from each alley and consumed it all in alley one would receive a score of four. Finally, at least in theory, animals could score a 0 , if they would not eat any of the baits. For consecutive days where bait was not presented in earlier alleys due to prior performance, the animal will automatically receive the value of each alley where bait is not presented.

\section{Results}

\section{Latency to Alley Four}

A mixed factorial ANOVA was conducted with time (days 1-6) as the repeated measure and genotype $\left(\mathrm{SERT}^{+/+}, \mathrm{SERT}^{+/-}, \mathrm{SERT}^{-/}\right.$) as the between groups measure to examine the effect of day and genotype on latency to enter alley 4 (figure 2). For the effect of days, Mauchly's test of sphericity determined that the assumption of sphericity was violated, thus Greenhouse-Geisser correction was used. A significant main effect of day was found, $[F(2.72,62.65)=8.46, p<.001]$, as well as a significant main effect of genotype was uncovered; $[F(2,23)=3.66, p=.042]$. Most importantly, however, no significant interaction between day and genotype was found through this analysis. Follow-up pairwise comparisons were performed to determine the source of the genotype difference. A significant difference was found between SERT ${ }^{+/+}$and SERT ${ }^{-/-}(p=.018)$, and between SERT ${ }^{+/-}$and SERT ${ }^{-/-}(p=.030)$. As figure 2 shows, while there was a significant reduction in latency over the trials, $\mathrm{SERT}^{-/-}$had significantly longer latencies throughout the experiment.

To determine the consistency of the anxiety measured over days, Cronbach's alpha was analysed. While less conventional than a simple correlation, it was deemed theoretically more valid 
(Santos, 1999). Performance on each day of testing was considered a scale item, thus Cronbach's alpha could be computed between latency to alley four at days 1 through 6 . Scores for latency to alley 4 showed acceptable internal consistency $(\alpha=.73)$.

To validate the mSAT as measure of anxiety, as opposed to a measure of exploratory behaviour, the association between latency scores and the percentage of 50khz USVs was examined. A two-tailed Pearson's correlation coefficient was computed between latency to alley four and the percentage of $50 \mathrm{khz}$ calls to examine the association between these variables. As predicted, a significant negative correlation was uncovered $[r(160)=-.28, p<.000]$, suggesting a negative association between these variables (see figure 3).

Figure 2. Latency to enter alley four over the course of the experiment

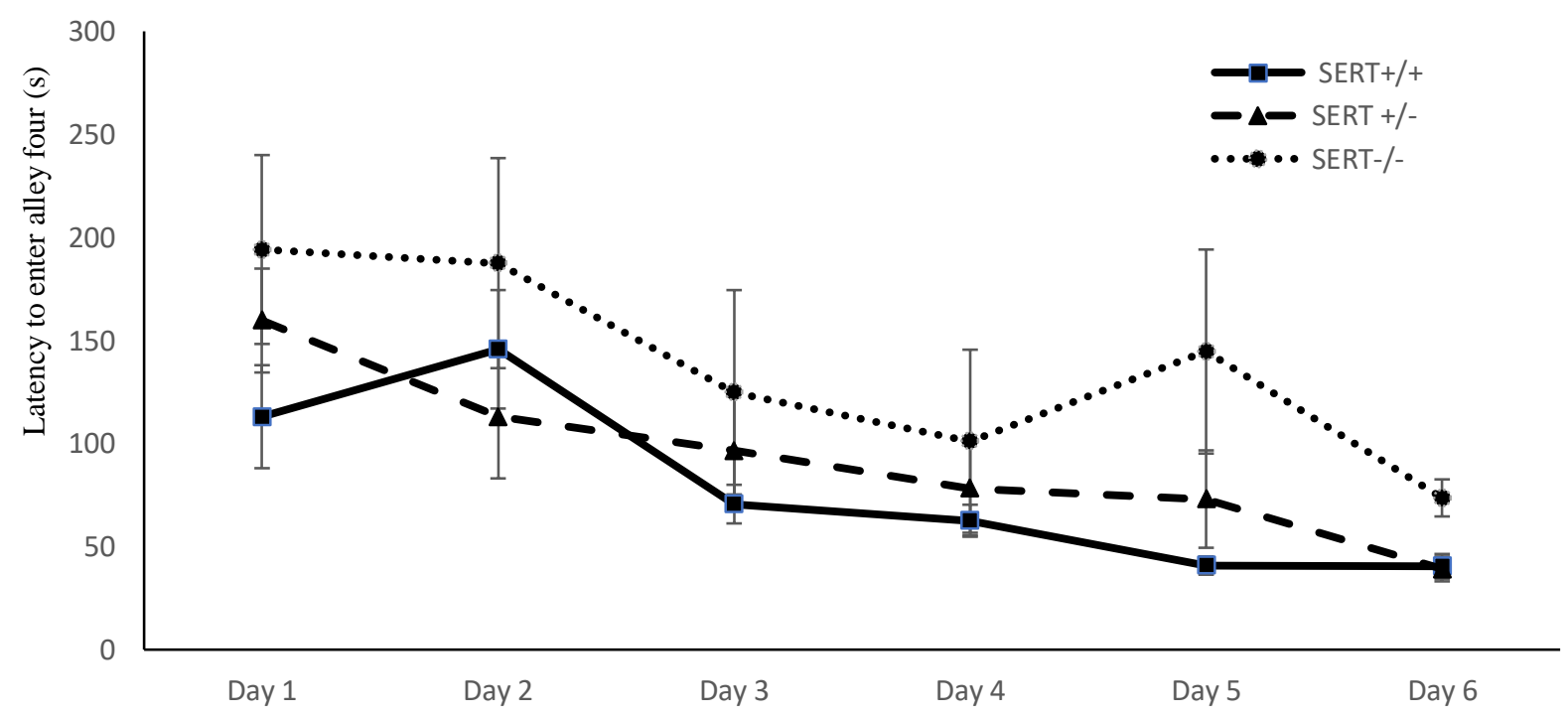

Figure 2. Means and \pm SEM of the latency to enter alley four across the six trial days (trail duration 600 seconds). Trial days are presented along the $x$ axis, while the latency in seconds is depicted along the y axis. SERT ${ }^{+/+}$are represented by the solid line, SERT $^{+/}$are represented by the dashed line, while the dotted line indicates the SERT ${ }^{-/}$group. A significant main effect of day $(p<.001)$ and genotype were uncovered $(p=.042)$, no further effects or interactions were uncovered. No interaction was observed. SERT-/- were found to show increased latency scores when compared to SERT ${ }^{+/+}(p=.018)$, and SERT ${ }^{-/-}(p=.030)$ groups, irrespective of day. 
Figure 3. Correlation between latency to alley four and the percentage of $50 \mathrm{khz}$ calls

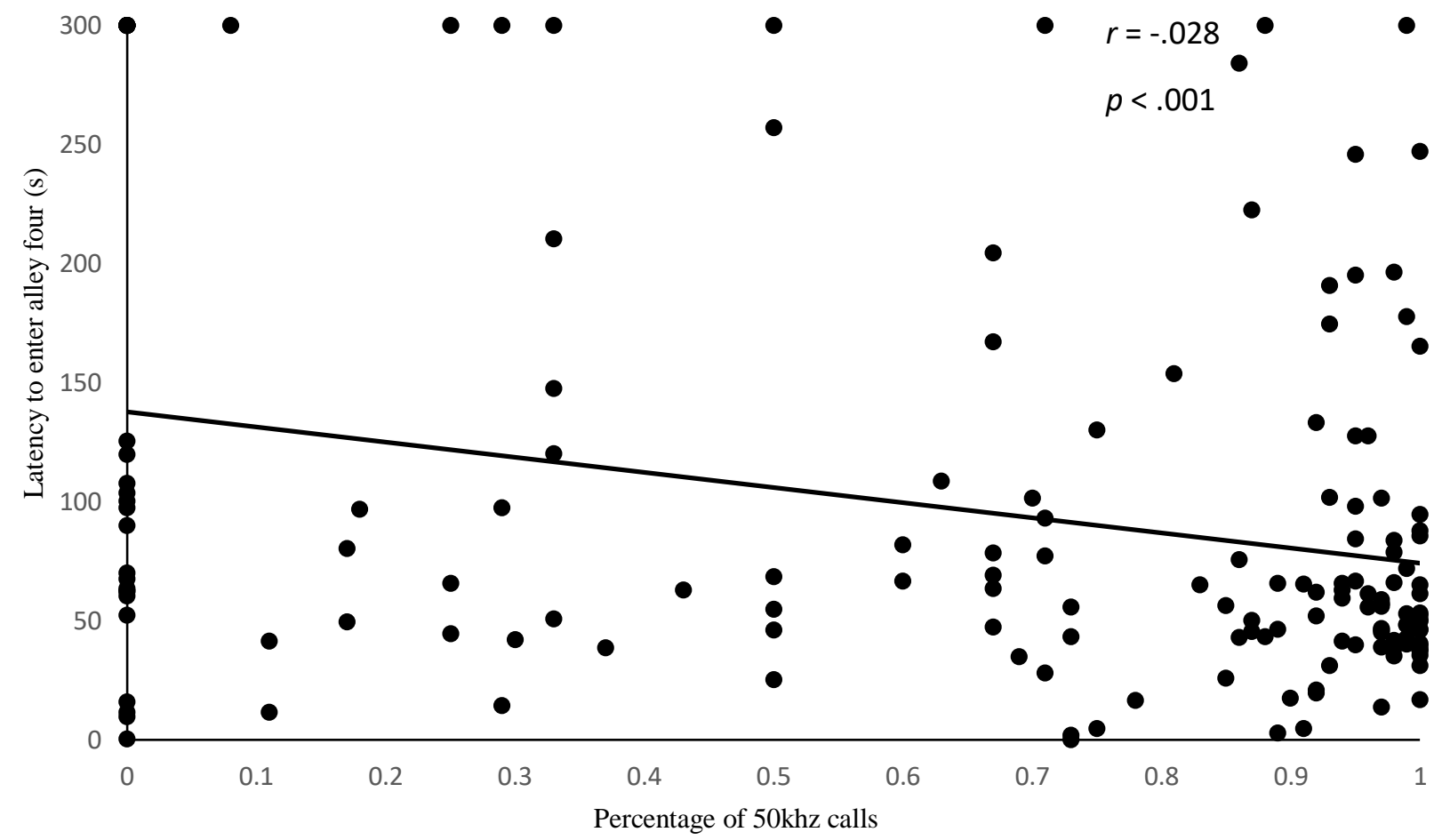

Figure 3. A scatterplot depicting the correlation between latency to enter alley four (s) and the percentage of $50 \mathrm{khz}$ anticipatory calls. A significant negative association is depicted by the trend line $(p<.001)$.

\section{Cumulative Duration in Alley Four}

Similarly to the prior analyses, a mixed ANOVA was conducted with time (days 1-6) as the repeated measure, and genotype $\left(\mathrm{SERT}^{+/+}, \mathrm{SERT}^{+/}, \mathrm{SERT}^{-/}\right)$as the between groups measure examining the effect of genotype and day on the cumulative duration spent in alley four. The assumption of sphericity was violated as determined by Mauchly's test of sphericity, thus the Greenhouse-Geisser correction was used for the following analyses. A significant main effect of day was observed $[F(2.77,63.58)=12.76, p<.001]$, as well as a marginally significant main effect of genotype was uncovered $[F(2,23)=2.98, p=.071]$, again, no interaction between genotype and trail day was observed (see figure 4). While the main effect of genotypes just missed significance, given the previous results with latency to alley 4, we followed up with a pairwise comparisons, which revealed significant difference between SERT ${ }^{+/+}$and $\mathrm{SERT}^{-/-}(p=.035)$, and between SERT $^{+/-}$and SERT ${ }^{-/-}$were observed $(p=.041)$, but not between SERT ${ }^{+/+}$and SERT ${ }^{+/-}$. Inspection 
of figure 4 showed that $\mathrm{SERT}^{-/-}$spent less time in alley four compared to $\mathrm{SERT}^{+/+}$and $\mathrm{SERT}^{+/-}$, irrespective of the effect of day.

To determine whether the same construct was assessed across all days, duration spent in alley four on each day was considered a single item, thus allowing for the analysis of reliability over time through the computation of a Cronbach's alpha $(\alpha=.86)$. Internal consistency of days one through six are considered high. Finally, a two-tailed Pearson's correlation revealed a significant positive correlation between duration in alley four and the percentage of high frequency calls $[r(160)=-.28, p<.000]($ see figure 5$)$.

Figure 4. Duration spent in alley four over the course of the experiment

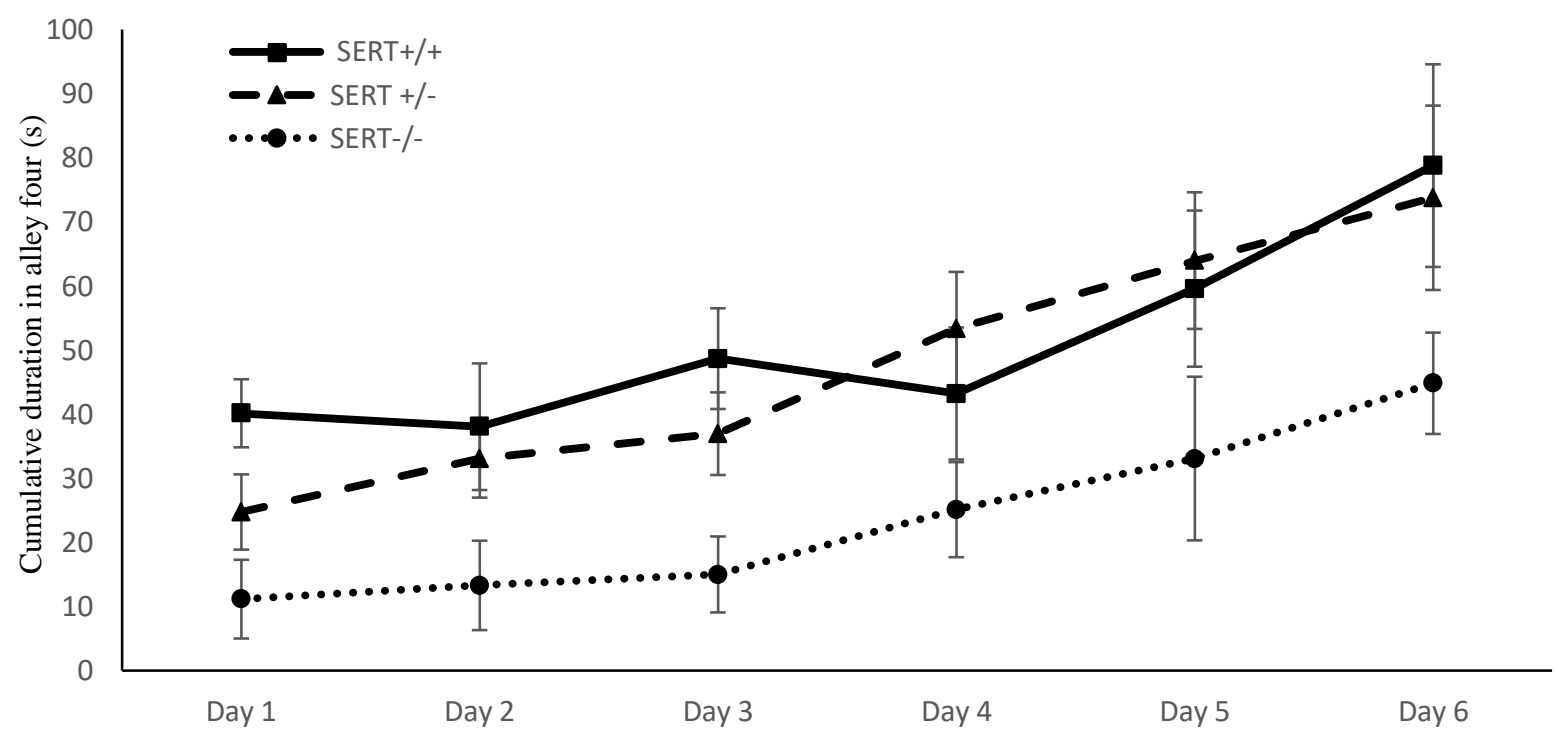

Figure 4. Means and \pm SEM of the cumulative duration spent in alley four across the six trial days (trail duration 600 seconds). Trial days are presented along the $x$ axis, while the duration in seconds is depicted along the y axis. SERT ${ }^{+/+}$are represented by the solid line, $\mathrm{SERT}^{+/-}$are represented by the dashed line, while the dotted line indicates the SERT ${ }^{-/}$group. Follow-up pairwise analyses indicated that the $\mathrm{SERT}^{-/}$group showed a reduction in duration spent in alley four when compared to SERT ${ }^{+/+}(p=.035)$, and $\mathrm{SERT}^{-/-}(p=.041)$ groups, irrespective of day. 
Figure 5. Correlation between duration in alley four and the percentage of 50khz calls

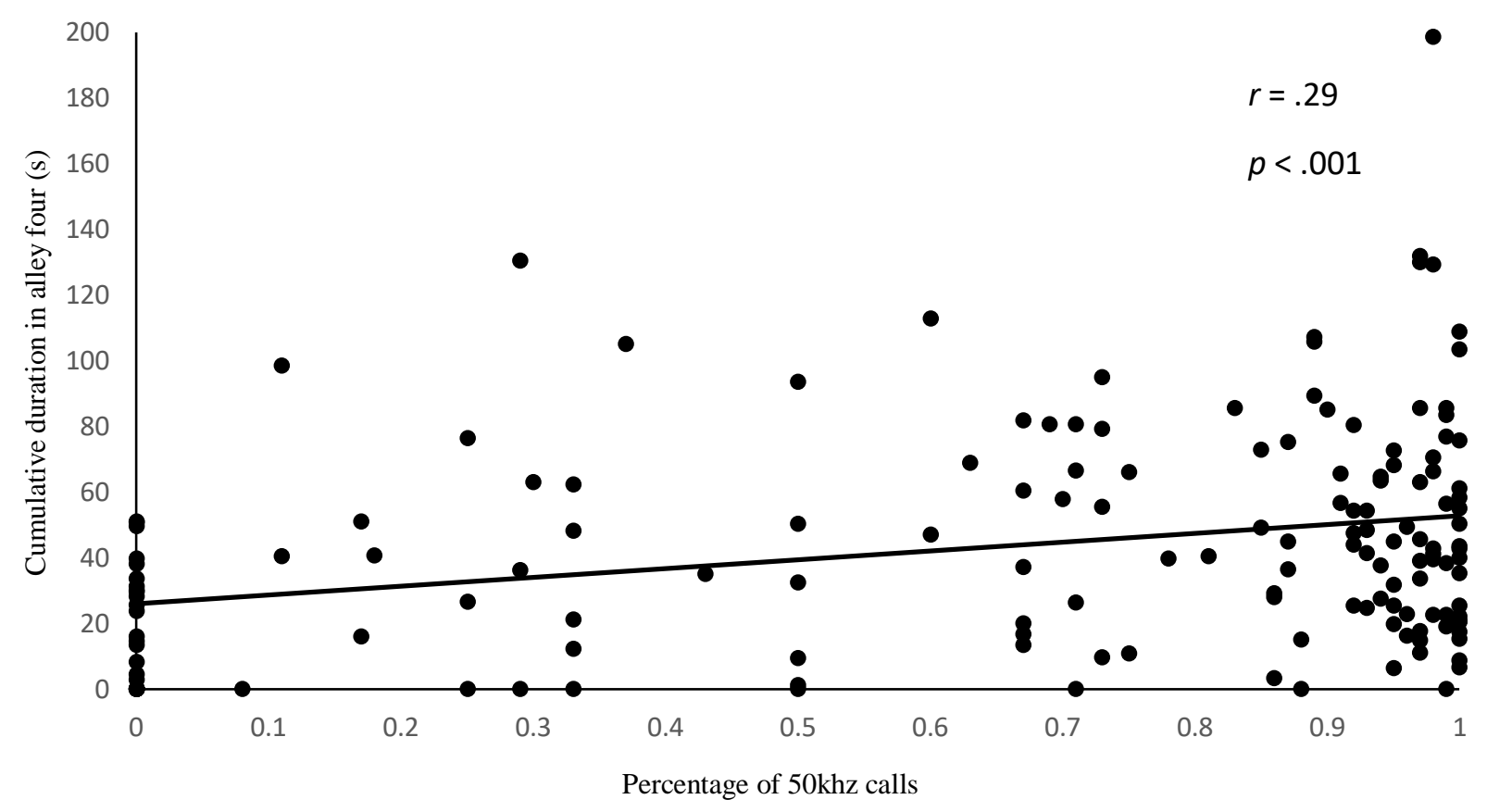

Figure 5. A scatterplot depicting the correlation between cumulative duration spent in alley four (s) and the percentage of $50 \mathrm{khz}$ anticipatory calls. A significant positive correlation is depicted by the trend line $(p<.001)$

\section{Retrieval Behaviour}

In addition to the classical parameters of latency and duration, we also evaluated a novel variable, namely retrieval behaviour, which indicates whether and where the animals consumed their food. As discussed in the material and methods section, higher retrieval scores are indicative of lower anxiety.

As with the previous two parameters, we first studied the effects of day and genotype on retrieval behaviour. A mixed ANOVA with time (days 1-6) as the repeated measure, and genotype $\left(\mathrm{SERT}^{+/+}, \mathrm{SERT}^{+/-}, \mathrm{SERT}^{-/}\right)$as the between groups measure was conducted. As determined by Mauchly's test of sphericity the assumption of sphericity was violated, therefore the GreenhouseGeisser correction was used in the following analyses. In line with the previous measures, a significant main effect was observed for day $[F(3.45,21.61)=6.21, p<.001]$, and genotype was identified $[F(2,22)=14.16, p<.001]$, while no significant interaction was observed (figure 6 ). 
Pairwise comparisons identified significant differences between all genotype SERT ${ }^{+/+}$and $\mathrm{SERT}^{+/-}$ $(p=.034), \mathrm{SERT}^{+/+}$and SERT ${ }^{-/-}(p<.001), \mathrm{SERT}^{+/-}$and $\mathrm{SERT}^{-/-}(p=.002)$, Figure 10 shows that $\mathrm{SERT}^{-/-}$had the lowest retrieval scores, and SERT ${ }^{+/+}$had the highest, with $\mathrm{SERT}^{+/-}$rats having intermediate values.

As with prior behavioural measures, a Cronbach's alpha was computed to determine if the retrieval behaviour at time one was a constant predictor of retrieval behaviour over time, thus implying the measurement of the same construct. Supporting this, a high reliability was observed $(\alpha=.86)$.

A two-tailed Pearson's correlation was computed between retrieval data and latency to enter alley four, revealing a significant negative association $[r(160)=-.39, p<.001]$ (see figure 7 ), while a significant positive relationship was uncovered between retrieval data and the cumulative duration spent in alley four $[r(160)=.43, p<.001]$ (see figure 8$)$. 
Figure 6. Retrieval scores over the course of the experiment

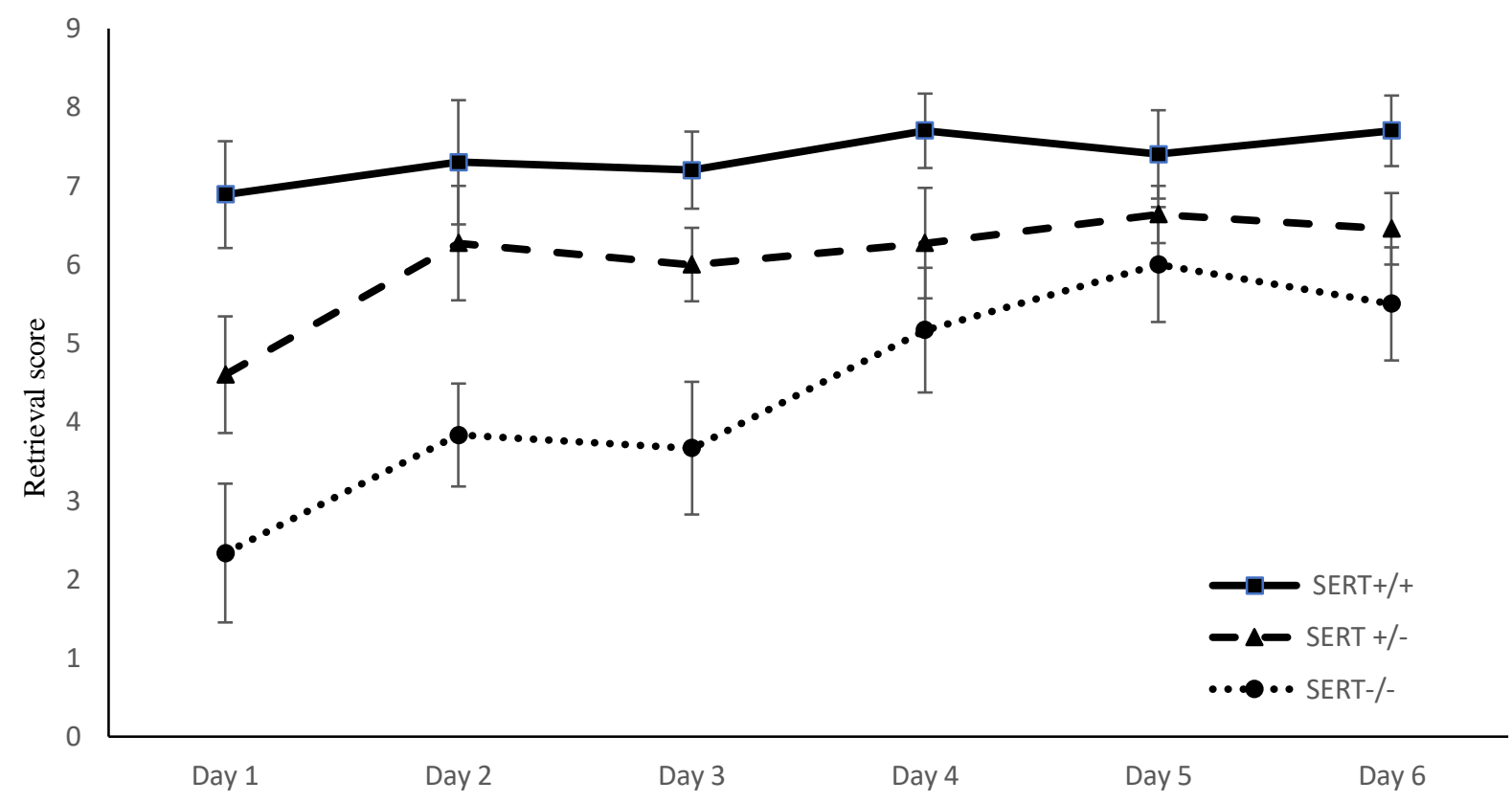

Figure 6. Means and \pm SEM for the retrieval scores across the six trial days. Trial days are presented along the $x$ axis, while the retrieval scores along the $y$ axis. SERT $^{+/+}$are represented by the solid line, SERT ${ }^{+/}$are represented by the dashed line, while the dotted line indicates the SERT $^{-/}$group. A significant main effect of day $(p<.001)$ and genotype were uncovered $(p<.001)$, while no interaction was observed. Pair wise analyses revealed that all groups significantly differed irrespective of day, with $\mathrm{SERT}^{-/}$showing reduced retrieval scores compared to both the $\mathrm{SERT}^{+/+}$ $(p<.001)$ and SERT ${ }^{+/-}(p=.002)$, furthermore, the SERT ${ }^{+/-}$showed a reduced retrieval score in comparison to the SERT $+/+$ group $(p=.034)$. 
Figure 7. Correlation between retrieval scores and latency to enter alley four

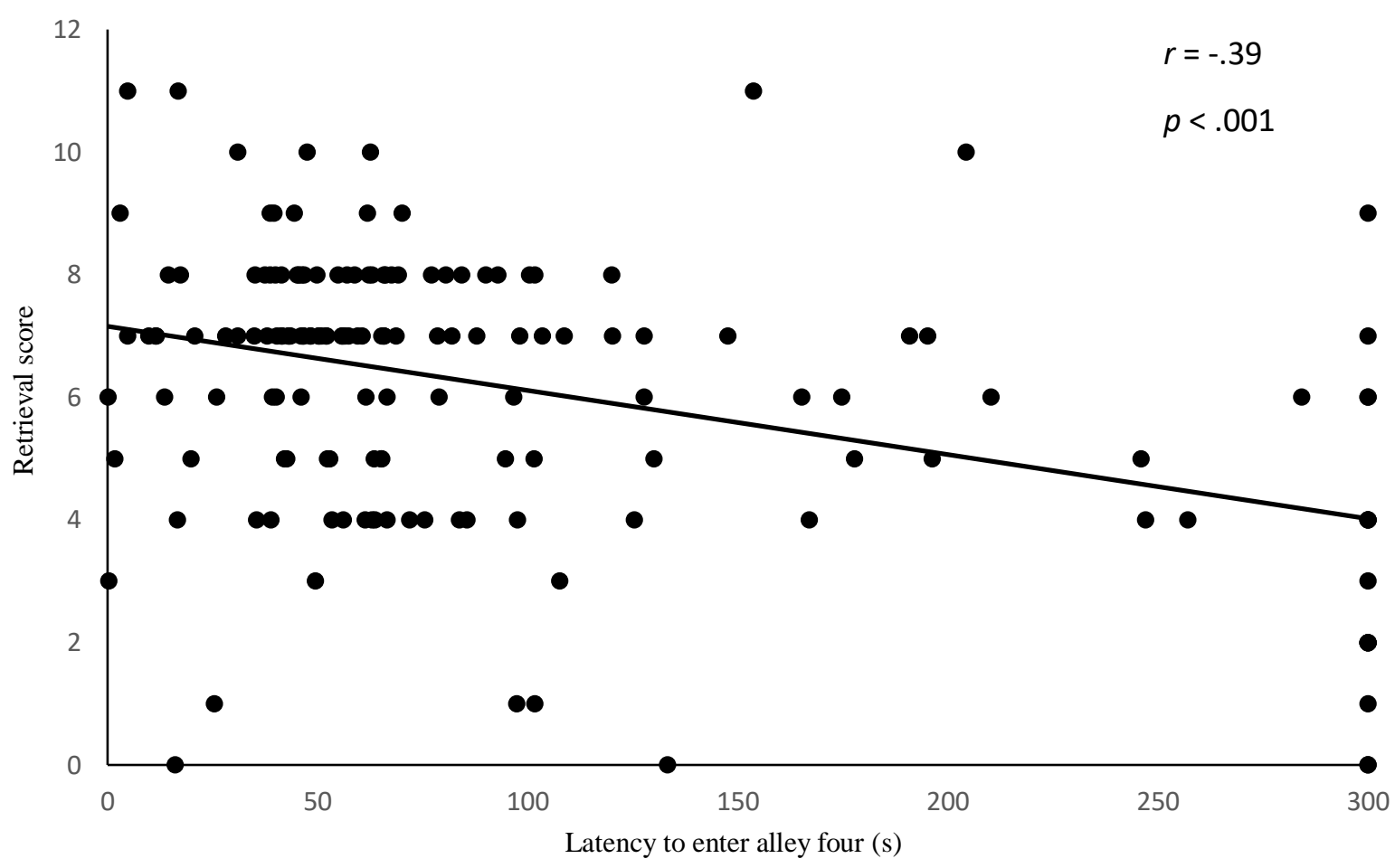

Figure 7. A scatterplot depicting the correlation between retrieval scores and latency to enter alley four (s). A significant negative correlation is depicted by the trend line $(p<.001)$

Figure 8. Correlation between retrieval scores and duration spent in alley four

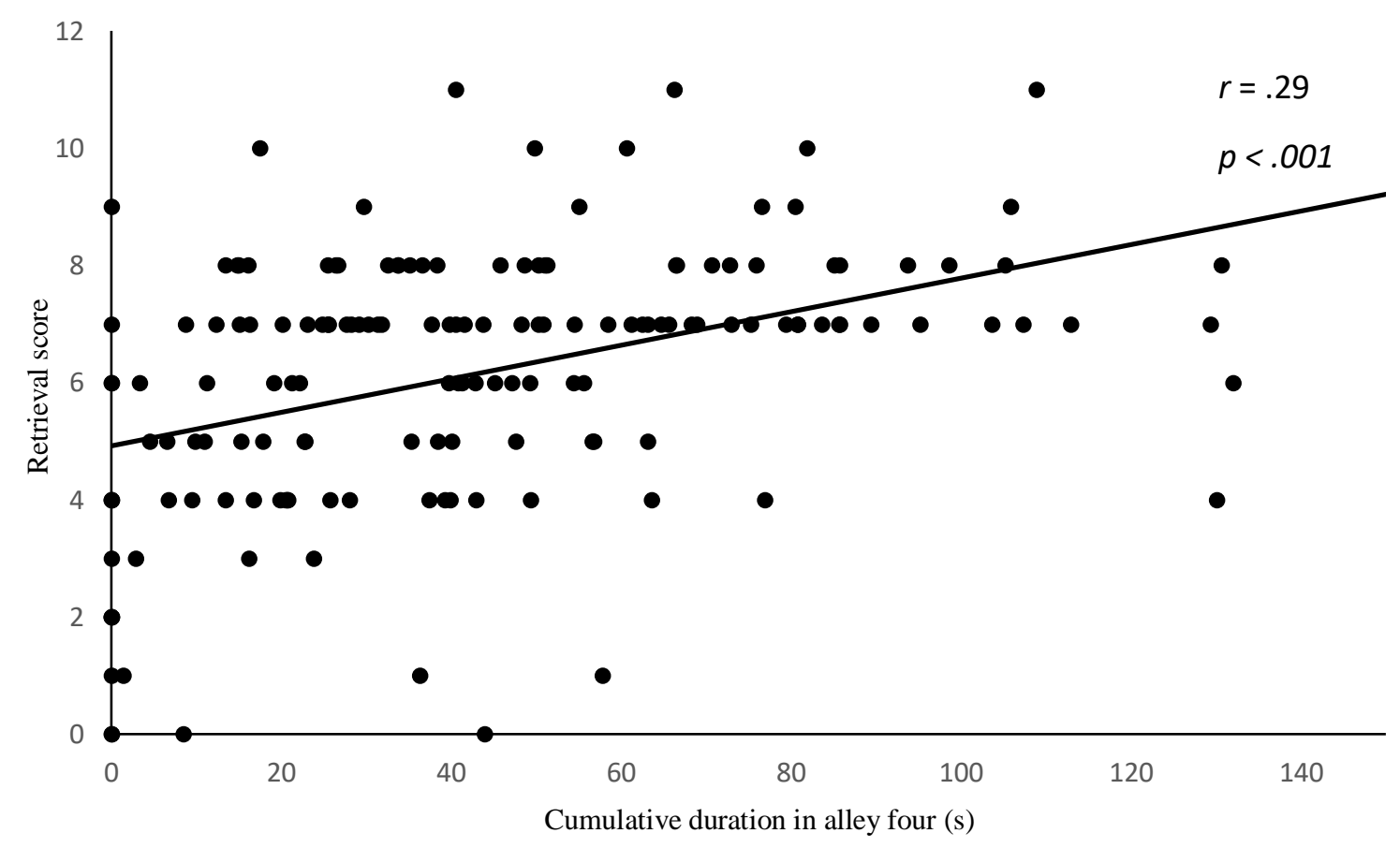


Figure 8. A scatterplot depicting the correlation between retrieval scores and the cumulative duration spent in enter alley four (s). A significant positive correlation is depicted by the trend line $(p<.001)$

\section{Discussion}

The SAT is still a relatively novel test for assessing anxiety, particularly when compared to the elevated plus maze but also with other classic anxiety paradigms (open field, light dark box etc.). In the present experiment we aimed to further improve on the SAT, and our results show that (1) anxious behaviour can be reliably recorded for at least 6 sessions; (2) there is a remarkable consistency between different measures of anxiety (latency to enter alleys four, food retrieval behaviour, USVs) and (3) rats with a genetically compromised SERT activity show trait dependent anxious behaviour. Thus, our findings not only provide support for the mSAT as a valid model for measuring trait anxiety, but further provides evidence that the validity of the SERT knockout animals as an animal model of trait anxiety. Overall, our hypotheses that there would be a gene dose effect on anxiety were mostly supported as significant differences between $\mathrm{SERT}^{+/+}$and $\mathrm{SERT}^{-/}$groups were observed in measures of latency to alley four, duration in alley four, and retrieval scores. However, $\mathrm{SERT}^{+/+}$and $\mathrm{SERT}^{+/-}$groups did not significantly differ over time in duration and latency measures, indicating if differences in anxiety are present between these genotypes, they may have been too subtle to detect with these more classical parameters. Similar results have been observed with anxious behaviours between SERT ${ }^{+/+}$and SERT ${ }^{-/+}$mice rarely being detected, a finding which is particularly robust in males (Ansorge, Zhou, Lira, Hen, \& Gingrich, 2004; Holmes, Yang, Lesch, Crawley, \& Murphy, 2003). Likewise, in a previous paper from our group, no genotype differences were found between $\mathrm{SERT}^{+/+}$and $\mathrm{SERT}^{+/}$in the elevated plus maze and the novelty suppressed feeding task (Ellenbroek, August, \& Youn, 2016).

Intriguingly, $\mathrm{SERT}^{+/-}$rats different significantly from $\mathrm{SERT}^{+/+}$rats in retrieval scores, suggesting this may be a more sensitive measure of anxiety. Retrieval scores are a novel measure 
where resources are plentiful to overcome periods of scarcity. Given that food deprivation induces an artificial resource scarcity, the animal is more likely to engage in pilfering and collection for consumption purposes, however, this scarcity increases competition and predation, thus animals are more likely to return to a safe location prior to consumption (Verdolin, 2006). While seemingly adaptive, complete retraction from an area containing resources increases the probability that these areas will be pilfered prior to returning. Due to this interplay between resource competition and predation, the most adaptive behaviour would be a partial retraction to a nearby lower risk location, allowing for the consumption of collected resources and a rapid return to continue collection, a balance between risk and reward (Lima \& Dill, 1990). This behaviour has been experimentally tested in jackrabbits (Longland, 1991), an animal facing similar predation and competition to rodent species (Erlinge et al., 1983). Furthermore, it should be noted that these retrieval and hoarding behaviours are not moderated by the direct presence of a predator or its scent, rather, through indirect cues such as increased exposure (Verdolin, 2006), thus there is no need to introduce direct predation cues into the SAT to induce these natural behaviours. These behaviours are mirrored using the mSAT, whereby less anxious animals $\left(\mathrm{SERT}^{+/+}\right)$would only partially retract to a location deemed relatively safe, while anxious $\left(\mathrm{SERT}^{-/}\right.$) animals deem these areas as too stressful, thus more often resulting in a complete retraction for consumption. While, as mentioned above, our data suggests that retrieval behaviours provide a more sensitive measure of anxiety-like behaviours, further validation is clearly required using other (genetic) models of anxiety, and the effects of anxiogenic and anxiolytic pharmacological interventions.

The present study also included USVs as they have been considered a relatively accurate representation of the emotional state of the animal, with calls within the 50khz range being indicative of a positive emotional state, while a negative emotional state being represented by calls within the $22 \mathrm{khz}$ range. Due to the serotonergic system being implicated in both exploratory and anxiety like behaviours through different receptor subtype combinations (see Gaspar et al, 2003 for a full review), and exploratory behaviours being heavily involved in current behavioural 
measures of anxiety, the inclusion of a measure of internal state allows for the differentiation of the mechanisms underlying the observed behaviours. The inclusion of anticipatory USVs provides further validity to the measure as the anticipation of exposure to a stressful stimulus would result in an increased proportion of $22 \mathrm{khz}$ calls and/or a reduction in the total number of $50 \mathrm{kHz}$ calls, while the inverse would be expected in anticipation of a positive environment or stimulus. Unfortunately, we observed a large degree of variation between animals in the total number of $50 \mathrm{khz}$ and $22 \mathrm{khz}$ call. Furthermore, some animals presented no $50 \mathrm{khz}$ calls on day one, while other animals showed no $22 \mathrm{khz}$ calls on the first day of the experiment. As a result, we were unable to measure the relative increase in these calls over time. As discussed in the material and methods section, we opted for a ratio between $50 \mathrm{khz}$ and $22 \mathrm{kHz}$ calls. This, we reasoned would allow to detect a difference between positive (higher 50khz percentage) and negative (lower 50khz percentages) internal emotional states. Thus it was hypothesised that the percentage of $50 \mathrm{khz}$ calls would be positively associated with the duration of time spent in, but negatively with the latency to enter alley four. As hypothesised, percentage of 50khz calls was positively correlated with the duration spent in alley four, while inversely correlated with latency to alley four. Given these associations, anticipatory USVs improve the validity of the SAT as the observed behaviours are associated with the predicted internal state of the animal.

Finally, the use of the modified version of the SAT proved useful as a behavioural paradigm to assess trait anxiety-like behaviours which persisted over multiple consecutive testing days, thus overcoming the OT'T problem. As figures 2, 5 and 10 indicate, and the statistics confirm, there was no interaction between genotype and day, thus confirming that the anxious phenotype of the rats with a genetically compromised SERT activity is maintained throughout testing. Some studies have suggested that through repeatedly testing anxiety behaviours in the same subject alters the mechanisms underlying the behaviour, thus only the first test session is truly indicative of anxiety-like behaviours (Carobrez \& Bertoglio, 2005). While this likely explains the OTT problem previously discussed, this effect was not observed in the present experiment. To further determine 
whether the behaviours examined at each time point were the same behavioural construct as observed on each other day, the Cronbach's alpha of each measure was calculated using each day as a 'scale item'. For each behavioural measure, there was a sufficient alpha value suggesting behaviours exhibited on each day were reflective of the same behavioural construct, suggesting no OTT or behavioural shift present.

As discussed earlier, depression and anxiety disorders have a very high comorbidity, the highest of any two psychiatric illnesses (Melartin et al., 2002). This is likely due to a significant overlap in the underlying mechanisms, with both disorders being associated altered amygdala response to emotional stimuli, and serotonergic system alterations (Munafò et al., 2008). Furthermore, the first line treatment for both disorders involves modulation of the serotonergic system through the pharmacological blockade of the serotonin reuptake transporter (Hirschfeld, 2001). The role of serotonin in emotion and anxiety is complex, however, of the 14 known receptor types, the $5-\mathrm{HT}^{1 \mathrm{~A}}$ receptor has been most consistently implicated in anxiety (Gaspar, Cases, \& Maroteaux, 2003). For example, genetic reductions of the $5-\mathrm{HT}^{1 \mathrm{~A}}$ receptor subtype leads to increased anxiety-like behaviours (Ramboz et al., 1998). Furthermore, reduced 5-HT ${ }^{1 \mathrm{~A}}$ binding has been shown to be reduced in humans with depression and anxiety (Molina et al., 2011). As discussed, the reduction in serotonin transporter expression results in a persistently increased anxiety behaviour which has been demonstrated through the present study, as well as prior research using classic anxiety paradigms (J. Olivier et al., 2008).

Human studies have frequently implicated the serotonergic system in psychiatric disorders, particularly depression and anxiety (Lesch et al., 1996), the inclusion of an animal model with a dysfunctional serotonergic system improves the translatability to the human condition. Thus, genetic associations between the 5-HTTLPR polymorphism and anxiety have often been reported, whereby reduced expression of the serotonin transporter (s-allelic variation) has been shown to be associated with increased anxiety like traits (Mazzanti et al., 1998; F. Murakami et al., 1999). 
Furthermore, reduced 5- $\mathrm{HT}^{1 \mathrm{~A}}$ receptor binding has been shown in depressed humans (Drevets et al., 1999), as well as those with anxiety disorders (Akimova, Lanzenberger, \& Kasper, 2009). Interestingly, in healthy subjects, those scoring higher in anxiety traits demonstrated a reduction in 5-HT ${ }^{1 \mathrm{~A}}$ binding (Tauscher et al., 2001). These findings in humans are mimicked in the $\mathrm{SERT}^{-/}$, whereby anxiety like behaviours have been consistently been reported, as well as a reduction in 5$\mathrm{HT}^{1 \mathrm{~A}}$ binding being observed (J. D. Olivier et al., 2008). These effects are not limited to the 5$\mathrm{HT}^{1 \mathrm{~A}}$ receptor, as $5-\mathrm{HT}^{2 \mathrm{~B}, 2 \mathrm{C}, 3 \& 4}$ receptor subtypes have been implicated in anxiety to some degree, in humans and animal models (Barnes \& Sharp, 1999). While brain imaging studies provide insight into the underpinnings of psychiatric disorders, post-mortem studies could provide a valuable source of information, however results from post-mortem studies in regards to anxiety and depression often yield inconsistent results (Hall et al., 1997; Hendricksen, Thomas, Ferrier, Ince, \& O’Brien, 2004; Rajkowska, 2000). These inconsistencies likely emerge from uncontrollable environmental factors and low sample size due to limited tissue availability, thus animal studies are required to further elucidate the mechanisms underlying these psychiatric illnesses.

Overall, these data suggest that the modified SAT represent a novel animal model for assessing trait anxiety. Given that anxiety is a chronic disorder in humans, and that as genetic reduction in SERT activity is a well-known risk factor for anxiety disorders (Savitz \& Ramesar, 2004), this suggest the modified SAT may represent a model with higher translational validity. Moreover, the combination of several different measures both traditional motor behaviours (latency to enter, time spent) and more novel (retrieval behaviour, USVs) may allow for detecting more subtle differences in anxiety between different animals, while at the same time maintaining internal consistency. However, despite the promising results, some considerations should be taken; Firstly, there is a large degree of variance within groups, particularly in the USVs. The origin of this variance likely emerged from the age range of the animals. While all animals were considered mature, those in the highest age bracket (PND90-100) clearly displayed lower baseline USVs. As a result, we decided to use proportional change from baseline, which provided a more consistent 
measure, in future studies, age should be considered a factor likely to impact the results, thus stricter age distributions, even within an adult sample should be considered. Despite this variance, significant results were observed, thus implying these results to be relatively robust. In addition, future studies should include pharmacological treatments to assess the (added) benefits of the additional measures (USVs, retrieval behaviour) and should incorporate female rats as well. As Olivier et al showed, female $\mathrm{SERT}^{-/}$, like their male counterparts also display a more anxious phenotype than female SERT ${ }^{+/+}$rats. 


\title{
CHAPTER FOUR
}

\author{
Experiment Three
}

Heart Rate Variability as a Potential Biomarker of Depressive

\author{
Disorders
}


The current basis of animal models of psychiatric disorders tends to be falling behind the latest clinical research which may underlie the lack of efficacious pharmacological treatments emerging in recent years Ellenbroek and Weiwen (2018). With the animal models lagging behind the current research advancements, false negative results may occur due to a lack of translational validity of our models (Kola \& Landis, 2004). Moreover, most animal models focus on behavioural read-outs, while integration of physiological aspects of psychiatric illnesses may provide a more accurate description of the illness and in turn enhance the reliability and validity of our animal models.

An association between MDD and cardiovascular disease (CVD) has repeatedly been shown with rates of depression in patients with CVD ranging between 20-40\% (Carney et al., 1987; Gonzalez et al., 1996). This association is troubling as depressed patients have reduced medication compliance (Keller et al., 2002) and have more difficulty coping with the stress of an illness (Blumenthal, Williams, Wallace, Williams Jr, \& Needles, 1982; Mohr, Goodkin, Gatto, \& Van Der Wende, 1997). The directional relationship of this association has been controversial with some proposing that depression is merely a response to the stress of having a significant illness (Musselman, Evans, \& Nemeroff, 1998), or that factors often associated with depression (anxiety, drug abuse, etc) mediate this relationship (Hayward, 1995). However, there is substantial evidence that depression itself is an independent risk factor of developing CVD (Aromaa et al., 1994; Everson et al., 1996; Musselman et al., 1998; Pratt et al., 1996; Wassertheil-Smoller et al., 1996). For instance, a meta-analysis involving over 120,000 subjects concluded that depression increased the risk of CVD by $80-90 \%$ (Nicholson, Kuper, \& Hemingway, 2006) Importantly, depression also increased the risk of cardiovascular mortality and contributed not only to the onset but also to the progression and prognosis of CVD. Thus the association between depression and CVD is often described as a downward spiral in which depression and CVD mutually reinforce each other (Penninx, 2017). 
The association between CVD and MDD has been investigated by Musselman et al. (1998), and they identified sympatho-adrenal hyperactivity, modifications in platelet receptors, reduced heart rate variability (HRV) and hyperactivity of the hypothalamic-pituitary-adrenocortical axis (HPA) as key elements in increasing CVD risk. One key element in the link between MDD and CVD is a decrease in HRV. HRV refers to the beat-to-beat variation in heart rate and is a measure of the interplay between the two arms of the autonomic nervous system: the parasympathetic and sympathetic nervous systems. The sympathetic nervous system is involved in increased activity of physiological systems in response to stress or the fight or flight response noted by (Cannon, 1911). The parasympathetic system plays a more regenerative role by reducing activity in these physiological systems. The balance between these systems is of crucial importance as increased sympathetic activity or decreased parasympathetic activity can reduce HRV, while, decreased sympathetic activity, and increase parasympathetic activity will result in increased HRV (Thayer, Yamamoto, \& Brosschot, 2010) (figure 1).

HRV, therefore, may not only represent an important link between MDD and CVD, as suggested by Musselman et al. (1998), but may actually be fundamentally related to the aetiology of the psychiatric symptoms seen in patients with MDD. Intriguingly, many mental and physical illnesses, aside from MDD, are also associated with a reduction in HRV, including schizophrenia, bipolar disorder and autism spectrum disorders (Boettger et al., 2006; Casanova et al., 2014; Licht, De Geus, Van Dyck, \& Penninx, 2009; Sevcencu \& Struijk, 2010) (Ellenbroek, Kidwell, ColussiMas, Youn, 2018). Before investigating the relationship between MDD and HRV and the possible underlying mechanisms behind this association, it is important to understand the measurement and analysis techniques most commonly used to assess HRV, and where possible, identify what these parameters indicate. 


\section{The Assessment of HRV}

A range of measures to investigate HRV have been compiled and summarized below to provide a simple and understandable overview of the parameters and the information they provide. These measures have been separated into linear and non-linear techniques. However, for a detailed explanation of these measures and parameters, we would recommend reading the paper by Cardiology (1996) and a more recent review which includes non-linear techniques by Acharya, Joseph, Kannathal, Lim, and Suri (2006).

In general, HRV parameters can be subdivided into linear and non-linear measurements. Linear measurements typically use the interval between two heart beats (the so-called R-R interval) to construct a linear association between variables. While these measures have dominated the field of HRV so far, it is increasingly recognized that the HRV signals are non-stationary and non-linear (Faes, Chon, \& Nollo, 2009). This has led to the introduction of a series of non-linear measurements, sometimes referred to as fractal indexes. Table 1 lists a large number of linear and non-linear measurement. In the next section, we will briefly summarize the relevant parameters for the present discussion.

\section{Linear Parameters}

Linear parameters of HRV are computed through linear algorithms, whereby graphing the function will produce a linear association between variables. These parameters can be subdivided into two main categories: Time-domain, and Frequency domain.

\section{Time-Domain}

Time domain measures are linear measurement of heart rate variability derived from the variation in the interval between subsequent R-waves from an ECG signal (see figure 1). These measures can again be subdivided into statistical and geometric measures; however, geometric measures make group comparisons difficult to ascertain. 


\section{Frequency Domain measures}

Frequency domain measures are computed via the decomposition of the waveform of ECG-RR intervals (interbeat interval or RRIs) and are often used in to better differentiate between the influence of the parasympathetic and sympathetic nervous systems. To examine frequency domain parameters, data needs to be transformed to produce a spectrum, thus, Fast Fourier transformation (FFT) is typically used. FFT decomposes the ECG trace into its component frequencies. This transformation is useful only if the signal is stationary, therefore may be incompatible if used to analyse non-stationary or transient signals. Following the required transformation of the data, analyses may be carried out using parametric or non-parametric analyses. Each parameter has been suggested to be indicative of aspects of autonomic activity. Traditionally two different bands (high and low frequency) have been studied, although more recently two additional bands have also gained attention.

\section{Non-Linear Measures}

Non-linear parameters of HRV refer to analysis techniques which account for more than merely the beat to beat variation, but rather attempt to capture a range of the dynamic processes at play within an organism. While linear methods are well understood and simple to compute, which has resulted in their popularity, they fail to account for the interplay between many interconnected systems within an organism. Non-linear parameters are less understood and more complex in nature, however, some key parameters used in HRV analysis will be simplified below in hopes to promote their use, which may lead to a greater understanding of their applications. 
Table 1.

\begin{tabular}{ll}
\hline Parameters & \\
\hline Linear measures (time domain) & Description \\
RR & The mean of all RR intervals \\
SDNN & SD of the normal to normal (R-R) intervals \\
RMSSD & Square root of the mean squared difference between successive RRs \\
NN50 & The number of successive RR intervals that differ by more than $50 \mathrm{~ms}$ \\
pNN50 & The percentage of NN50 \\
HRV triangular index & The integral of the RR interval histogram divided by the height of the histogram \\
TINN & Triangular interpolation of $\mathrm{N}-\mathrm{N}$ intervals. Calculation is based on taking the highest peak in the RR histogram and creating a triangle \\
& using the minimum squared difference \\
Linear measures (frequency domain) & Power band encompassing the $0.15-0.4 \mathrm{~Hz}$ range \\
HF & Power band encompassing the $0.04-0.15 \mathrm{~Hz}$ range \\
LF & Power band encompassing the $0.003-0.04 \mathrm{~Hz}$ range \\
VLF & Power band encompassing the range below $0.003 \mathrm{~Hz}$ \\
ULF & Total power within the HRV spectrum \\
TP & The ratio between LF and HF \\
LF/HF & A method of visually representing chaos as opposed to random noise, whereby each R-R interval is plotted against the previous interval \\
Nonlinear measures & in a scatterplot \\
Poincare plot & Approximate entropy is a method to quantify the predictability of fluctuations in a signal over time \\
& Detrended fluctuation analysis represents a modified root mean square type of analysis \\
ApEn &
\end{tabular}

Table 1. A brief description of the most frequently reported parameters of HRV:

ApEn, approximate entropy; DFA, detrended fluctuation analysis; HF, high frequency; HRV, heart rate variability; LF, low frequency; SDNN, SD of the normal to normal (R-R) intervals; TINN, triangular Interpolation of N-N intervals; TP, total power; ULF, ultra-low frequency; VLF, very low frequency. Adopted from Kidwell and Ellenbroek (2018)

Figure 1. The Brain-Heart association and ECG depiction

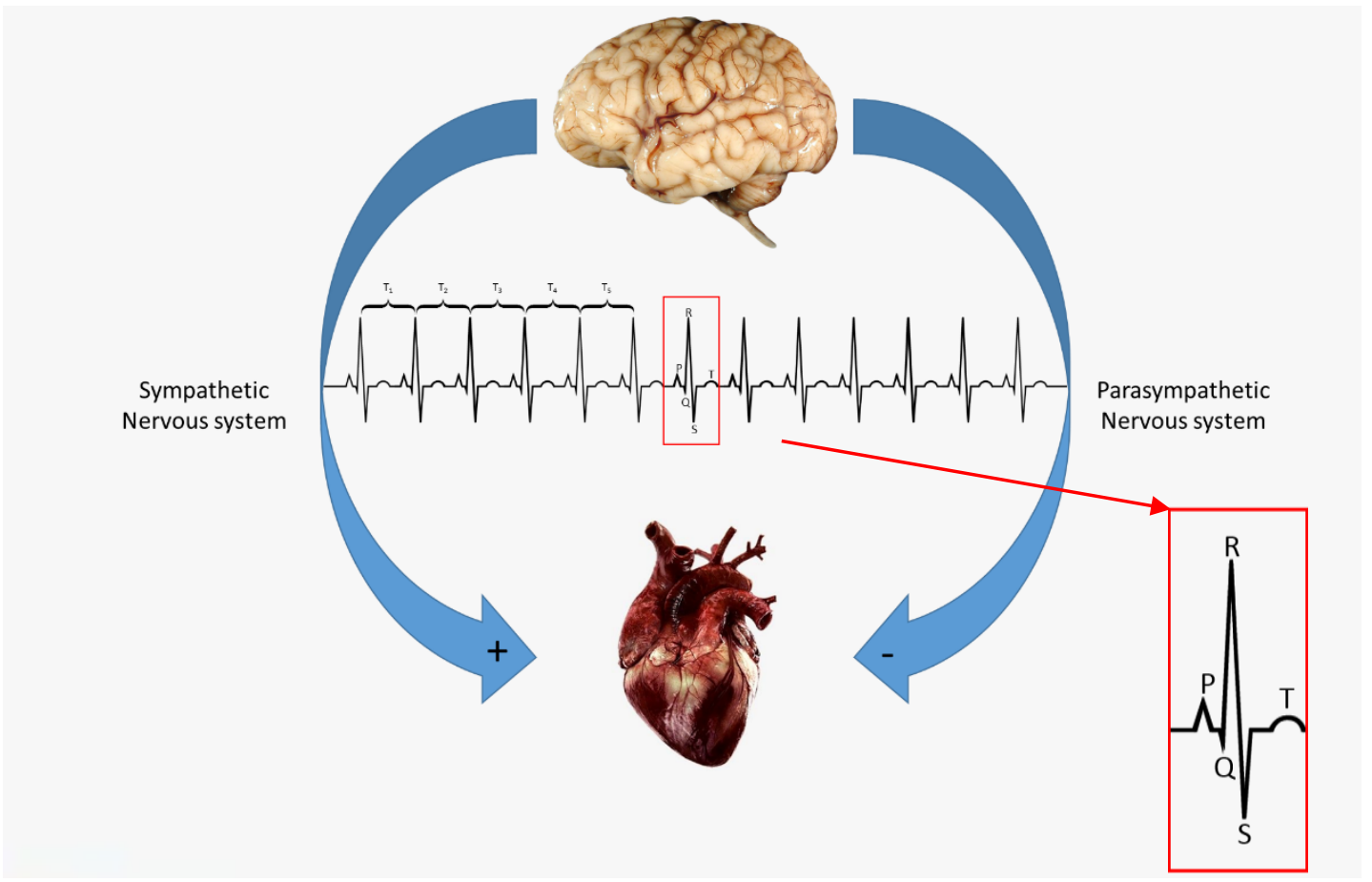


While the link between HRV and MDD is yet to be fully elucidated, a vast body of research has shown a relatively consistent association between reductions in HRV and MDD. Kemp, Quintana, Felmingham, Matthews, and Jelinek (2012) sought to examine this link in unmedicated patients with MDD. A significant reduction in HRV (reduced RMSSD, SDNN, \& HF, while increasing LF/HF ratio) was observed. The deficits observed were greater in the subset of patients with comorbid anxiety suggesting a compounding effect may be occurring. Similar results have been reported by van der Kooy et al. (2006) who examined HRV in elderly patients with depression: Reductions in HF, LF, RMSSD and SDNN, indicating reduced vagal tone and an overall reduction in HRV. This study also demonstrated that reductions in HRV are associated with depression, irrespective of age. In a meta-analysis conducted by Kemp et al. (2010), depression, symptom severity and treatments were examined in regards to their relationship with HRV alterations. Of the studies used, 11 examined depression and HRV directly, which included 401 depressed patients and 407 controls. Through this analysis, it was shown that depression was associated with decreased time domain measures of HRV, HF and several non-linear measures of $\mathrm{HRV}$, while the LF/HF ratio showed a significant increase. It should be noted that data were obtained from unmedicated participants (either drug naive, or following a washout period), and time domain measures were combined into a single grouping, while similarly, non-linear parameters were combined. The need to combine these measures highlights a lack of consensus over which measures should be reported across studies. More detailed studies provide evidence that symptom severity of patients with MDD is inversely correlated with HRV, especially with respect to the time domain parameters (such as RMSSD and SDNN) and HF, and possibly LF (Kemp et al., 2010; Yeh, Chung, Hsu, \& Hung, 2017). This would suggest a potential avenue of research into novel treatments of MDD with a focus on HRV outside of the effective yet invasive direct vagus nerve stimulation treatment for treatment-resistant MDD. Moreover, it suggests that reduced HRV may be a biomarker for MDD, or perhaps even be predictive for the development of MDD. Jandackova, Britton, Malik, and Steptoe (2016), examined participants HRV (SDNN, 
RMSSD, LF \& HF) and depressive symptomology using data from the Whitehall II study. Patients were assessed at two time points (10.5 years between assessments) and found that male patients who had lower HRV at time point 1 were less likely to report experiencing a depressive episode at time point 2, while females had a similar trend which failed to reach significance.

The exact relationship between MDD and HRV is still a matter of debate, as HRV is related to several important functional domains. Low baseline HRV, especially HF HRV has been associated with cognitive deficits, especially in tasks relying on the frontal cortex (Ottaviani et al., 2016). Additionally, reduced HRV (again predominantly HF-HRV) has been associated with emotional deficits and in particular to emotional inflexibility (Balzarotti, Biassoni, Colombo, \& Ciceri, 2017). In line with this, a recent neuro-imaging meta-analysis found a clear association between HRV and activation of the major neuronal areas involved in cognitive and emotional flexibility, most notably the anterior cingulate and prefrontal cortex, the insula and the amygdala (Ruiz Vargas, Sörös, Shoemaker, \& Hachinski, 2016). However, HRV is also influenced by many other processes, such as lifestyle and coping strategy, nutrition and personality trait (Appelhans \& Luecken, 2006; H. A. Young, Cousins, Watkins, \& Benton, 2017). Moreover, drugs, including antidepressant drugs are known to affect cardiovascular functioning.

Interestingly, the 5-HTTLPR genetic variation has been shown to affect HRV. Agorastos et al. (2014) examined the effect of cholecystokinin tetrapeptide (CCK-4), a substance known to produce panic attacks through its anxiogenic effects, on homozygous $\mathrm{S}$ allele carriers in comparison to homozygous L allele carriers. Not only did S/S carriers display a reduced baseline vagal tone $(\mathrm{HF})$, but following the administration of CCK-4, this reduction was further exacerbated. Furthermore, Yamakawa, Matsunaga, Isowa, and Ohira (2015) demonstrated S/S carriers show a reduction in LF/HF ratio in response to stress, which didn't reach significance, potentially due to the limited sample size. Finally, Crişan et al. (2009) showed that individuals carrying at least one copy of the $\mathrm{S}$ allele had a reduction in overall autonomic control, reduced 
vagal tone $(\mathrm{HF})$, and a marginally significant increase in the LF parameter of HRV. While the mechanism are yet to be fully understood, alterations in emotional regulation and coping styles in response to stress have been suggested (H. Murakami, Matsunaga, \& Ohira, 2009). As such, carriers of the s allele experience equivalent reductions in HRV in response to a stressor, as their $\mathrm{L}$ allele counterparts, however normalisation of HRV is delayed in the s allele carriers. This delayed normalisation of HRV has been observed in high and low anxiety line animals (HAB \& LAB), an animal model of anxiety selectively bred based on their performance in the elevated plus maze (Landgraf \& Wigger, 2002). HAB and LAB animals show equivalent HRV reductions in response to restraint, however, normalisation of HRV is significantly delayed in HAB animals in comparison to LAB animals (Carnevali \& Sgoifo, 2014).

Animal Models of Psychiatric Illness Integrating HRV

One of the most attractive features of HRV is that it can be assessed in humans and animals with very similar techniques. However, while several different devices exist for measuring heart rate, few studies have used these to assess HRV, and fewer yet have done so in animal models of psychiatric disorders (Ellenbroek et al., 2018).

Two core methods of examining HRV in animals exist, each with their own advantages and disadvantages, these are invasive, and non-invasive. Non-invasive methods involve the measurement of ECG signals through the skin of the subject, similarly to humans, however, these methods are plagued by movement artefacts, and may require an external device which may impact behaviour, although alternatives have also been developed. Invasive methods involve the surgical implantation of a telemetry device which sends ECG signals to an external receiver. This method provides the superior signal to noise ratio and does not impair free movement of the animal. However, in contrast to the non-invasive method, this is substantially more expensive and more challenging for the animals as it requires surgery. Moreover, given the size and weight of the telemetry probe, this technique cannot be used on very young animals. 
While the invasive method has excellent signal to noise characteristics, the signal can still be affected by movement artefacts, especially using the standard technique where the leads are connected close to the fore- and hindlimb muscles of the animals. However, Sgoifo et al. (1996), developed a technique which substantially reduced such artefacts, making the assessment of HRV much more reliable. This technique was also used in the present paper and will be described in more detail in the material and methods section.

\section{Depression models and HRV}

So far, only a few studies have investigated HRV in animal models for psychiatric disorders, and more specifically for depression. The Flinders line of rats were originally bred based for their cholinergic sensitivity. However, since then many studies have found that the Flinders Sensitive Line (FSL) show multiple behavioural and neurochemical similarities with major depression (Overstreet \& Wegener, 2013). Due to these similarities, Hildreth, Padley, Pilowsky, and Goodchild (2008), sought to investigate whether the FSL would exhibit similar HRV deficits observed in humans. Initially, ECG studies over 24 hours in FSL, FRL, and a standard Sprague Dawley line noted increase in HR in the FRL. More relevant for the present discussion, however, reduced HRV was noted in both the FSL (HF \& LF/HF ratio) and FRL (only HF) in comparison to the Sprague control group. The reduction in HRV observed in the FRL was suggested to be a direct result of the increased HR, while the FSL animals displayed reductions in HF and increased $\mathrm{LF} / \mathrm{HF}$ ratio independent of $\mathrm{HR}$ changes, suggestive of a reduced parasympathetic control, similar to that observed in depressed humans.

Another animal model of depression, the chronic mild stress paradigm (CMS), involves exposing a rat to a series of unpredictable stressors over a prolonged period of time (e.g. strobe lights, wet bedding, tilting the cage) which induces behavioural impairments associated with depressive disorders, such as anhedonia. Grippo et al. (2003) examined HRV of CMS animals following a 4-week recovery period. The authors measured HRV through SDRR, showing a 
significant reduction in those exposed to CMS. Interestingly, these cardiovascular changes were present despite the normalisation of sucrose preference and locomotor activity, indicating the impact of CMS on HRV are independent of the behavioural changes observed in response to stress (and may thus be a more sensitive measure of a negative emotional state).

Anxiety models and HRV

Animal models of anxiety have also been subjected to HRV analysis. The high anxiety behaviour (HAB) and low anxiety behaviour animals (LAB), selectively bred for their anxious phenotype in the elevated plus maze were used in a study by Carnevali and Sgoifo (2014) to examine resting HRV and HRV changes following the induction of stress through restraint. The HAB animals showed reductions in HRV (RMSSD, HF, \& TP) during rest, while the induction of stress through restraint showed a muted reduction of $\mathrm{HRV}$ in the HAB animals compared to LAB animals. This muted response is likely to be a result of the reduced vagal control of the HAB animals, which has been noted to be associated with reduced emotional regulation in human studies (Ellenbroek, et al., 2018).

While there are clear differences in HRV associated with animal models of psychiatric disorders, which improve the translational validity of the model, outside of these studies, the examination of HRV is often overlooked. Given the lack of HRV studies in more prevalent disorders, it is unsurprising that no known model of schizophrenia or autism have integrated HRV analysis to date (Kidwell \& Ellenbroek, 2018a).

Given the relationship between major depression and HRV on the one hand, and between HRV and the s-allele of the 5-HTTLPR, the purpose of the present study was to examine the impact of the genetic reduction of serotonin transporter expression on parameters of HRV. Given the prior literature, we would hypothesise that reductions will be observed across HRV parameters, primarily those modulated by parasympathetic inputs (RMSSD, HF, SDSD) in SERT ${ }^{-/}$rats 
compared to $\mathrm{SERT}^{+/+}$rats. In line with the previous chapters, we expected a gene-dose relationship with the $\mathrm{SERT}^{+/-}$showing an intermediate response.

\section{Method}

\section{Surgical equipment}

Standard surgical equipment is required for this procedure; however, some specialty equipment was used:

A blunt dissector and tight-fitting removable polymer cylindrical enclosure was used. This provides less tissue damage than other methods of blunt dissection. The polymer enclosure allows for the withdrawal of the blunt dissector and guidance of the lead to the desired location.

A 10-14 gauge needle hypodermic needle is required. The needle requires a lead to be easily guided through it, thus needs to be sufficient in size. This allows the tissue to close around the lead resulting in less tissue damage or risk of tearing.

A soft plastic drinking straw was modified to assist with the final lead placement. This was required to prevent internal damage caused by more rigid surgical equipment.

An implantable telemetry device (TA1 ICTA-F40; Data Sciences International) with compatible device reader pad and associated software (LabChart, ADInstruments).

\section{Surgical procedure}

The standard preoperative procedure was performed in preparation for surgery. A dose of $90 \mathrm{mg} / \mathrm{kg}$ ketamine $+9 \mathrm{mg} / \mathrm{kg}$ xylazine solution was administered intraperitoneally to induce anaesthesia and analgesia. Following the cessation of the paw reflex, ensuring sufficient anaesthesia, the ventral surface of the animal's neck, and the ventral abdominal were shaved using electric clippers. Exposed skin was sterilised using 70\% ethanol solution, betadine prior to 
transference to the surgical room. Finally, carprofen, a nonsteroidal anti-inflammatory drug (NSAID) was administered subcutaneously $(5 \mathrm{mg} / \mathrm{kg})$.

The implantation procedure was developed on the basis of Sgoifo et al. (1996). However, due to the limited detail presented in the procedural section, some modifications had to be established; firstly, the lead positive lead termination site was describe as being accessed by tunnelling the under the muscle, along the trachea, finally terminating in the anterior mediastinum, near the right atrium. During attempts to recreate this, the flexibility of the lead would result in an inability to breach into the anterior mediastinum and attempts to use more rigid guiding system often resulted in animals experiencing cardiac failure. To circumvent this, a small incision was created in the muscle surrounding the trachea, allowing the lead to be inserted alongside the trachea with less resistance. The modified straw guide was used to breach into the mediastinum, with the new angel created allowing a greater degree of control. Due to the novel entrance point of the positive lead, the anchoring points required modification. The lead was sutured to the muscle surrounding the trachea while closing this incision, while two further anchor points were created on the sternocleidomastoid muscle. Aside from these modifications, the remainder of the procedure remained the same, whereby the body of the telemetry device in inserted into the peritoneal cavity, with the negative lead being sutured to the dorsal surface of the xiphoid process.

Following the successful completion of the surgical procedure, 10ml Hartmans solution and $0.2 \mathrm{ml}$ of penicillin $(250,000 \mathrm{IU} / \mathrm{ml})$ was administered subcutaneously. Terramycin powder was puffed over the incision sites to prevent post-operative infections, following which, the animal was allowed to recover on a heating pad until fully mobile prior to being returned to their home cage. Following the surgery penicillin and carprofen were administered daily. Core body temperature was measured daily through the telemetry device as an early detection measure of infection. 
Animals used for this procedure were the same as used in experiment one, however, due to some health concerns presented by two animals within the four-month period between experiments, they were deemed unfit for surgery and were euthanized. Of the remaining animals, a total of 3 suffered from surgical or postoperative compilations and were rapidly euthanized. Therefore, 27 animals were used for data collection in this experiment. Due to RFID malfunctions, likely from the magnetic switch of the telemetry device, and the use of a blinded experimental design, four animals were unable to be identified. Therefore, eight SERT ${ }^{+/+}$, eight $\mathrm{SERT}^{+/-}$, and seven $\mathrm{SERT}^{-/}$, were used in the final analysis. Animals were housed in the same conditions as in prior experiments, with single housing being required due to experimental procedures. All procedures were in accordance with the Animal Welfare Act 1999, with all procedures being approved by the VUW Animal Ethics committee (AEC number 24384).

\section{Experimental setting}

The present experiment took place in the same room as experiment one, however separate cabinets were used which contained one receiver per cabinet (maximum of 8 testing session). The room was constantly dark to match the activity period on which testing took place, with the exception of the 24-hour testing sessions, in which lights were manually adjusted to match the reverse cycle present in the housing facility. As we only had limited numbers of telemetry probes, the experiments took place in two separate cohort.

\section{Procedure}

In each of the cohort, heart rate was measured during three a baseline 24-hour recording was collected, whereby the animal in its regular housing cage was transported to the experimental room and placed on the receiver pads. After 8 animals had been transferred into the experimental room, the Labchart software was started and each animal had their telemetry device turned on 
with a magnet without any handling. Data were collected until the same time the following day, at which point the remaining animals were cycled through the same process. Following data collection, all animals were euthanized, and the telemetry probes removed and cleaned for renewed use.

ECG data obtained in this experiment were analysed using Labchart (AD Instruments), using the HRV add-on module. The VLF window was predefined following FFT as 0-0.04 Hz, LF as $0.04-0.15 \mathrm{~Hz}$, and $\mathrm{HF}$ as $0.15-0.45 \mathrm{~Hz}$. Percentage values are used to compensate for variable impedance, commonly observed within electrophysiology data. The HF percentage (HF/TP) was used as $\mathrm{HF}$ is the most reported frequency parameter within the depression literature, while being a near pure measure of parasympathetic modulation of the cardiovascular system. Furthermore, RMSSD was used as a generalised measure of HRV, while being the most frequently reported time domain parameter within the depression literature.

Comparisons of HRV are very dependent of the quality of the underlying ECG signal, thus equivalence between groups needed to be established. Labchart's standard HRV detection settings (1.8 complexity maximum; 200ms R-R interval maximum) resulted in significantly different proportion of detected beats between groups, suggesting important differences in the morphology of the ECG between the genotypes (see also below). Therefore, we adjusted the criteria to a 3.0 complexity maximum and a 300ms R-R interval maximum. These new criteria allowed the assumption of group equivalence to be met, as there were no differences between the percentage of included beats anymore $(F(2,858)=46.59, p<.153)$. HRV parameters were computed in 5 minute bins of ECG outputs for the 24-hour duration of the experiment (288 per animal). The $\mathrm{HF} \%$ and RMSSD was calculated for each 5-minute segment of data, then averaged for each hour, creating a stable average $\mathrm{HF} \%$ and RMSSD value for each animal, per hour over the course of 24 hours. 


\section{Results}

Means and standard deviations were computed for the average hourly RMSSD score in the $\mathrm{SERT}^{+/+}, \mathrm{SERT}^{+/}$, and $\mathrm{SERT}^{-/}$groups (see appendix 8). A mixed ANOVA was conducted with time (Hours 1-24) as the repeated measure, and genotype $\left(\mathrm{SERT}^{+/+}, \mathrm{SERT}^{+/-}, \mathrm{SERT}^{-/}\right.$) as the between groups measure examining the effect of genotype and time of day on RMSSD (Figure 2). Due to the violation of sphericity, the Greenhouse-Geisser correction was adopted for the following analyses. No significant main effects, nor interactions were observed, indicating that the effect of time and genotype have no effect on HRV as measured through RMSSD. To determine whether any effects were being masked by the number of levels, four blocks consisting of six hours each (hours 1-6, 7-12, 13-18, and 19-24) were produced and subjected to a mixed ANOVA, however, as with the prior analysis, this simplification produced no significant effects or interactions (Figure 3).

Figure 2. RMSSD over 24 hours

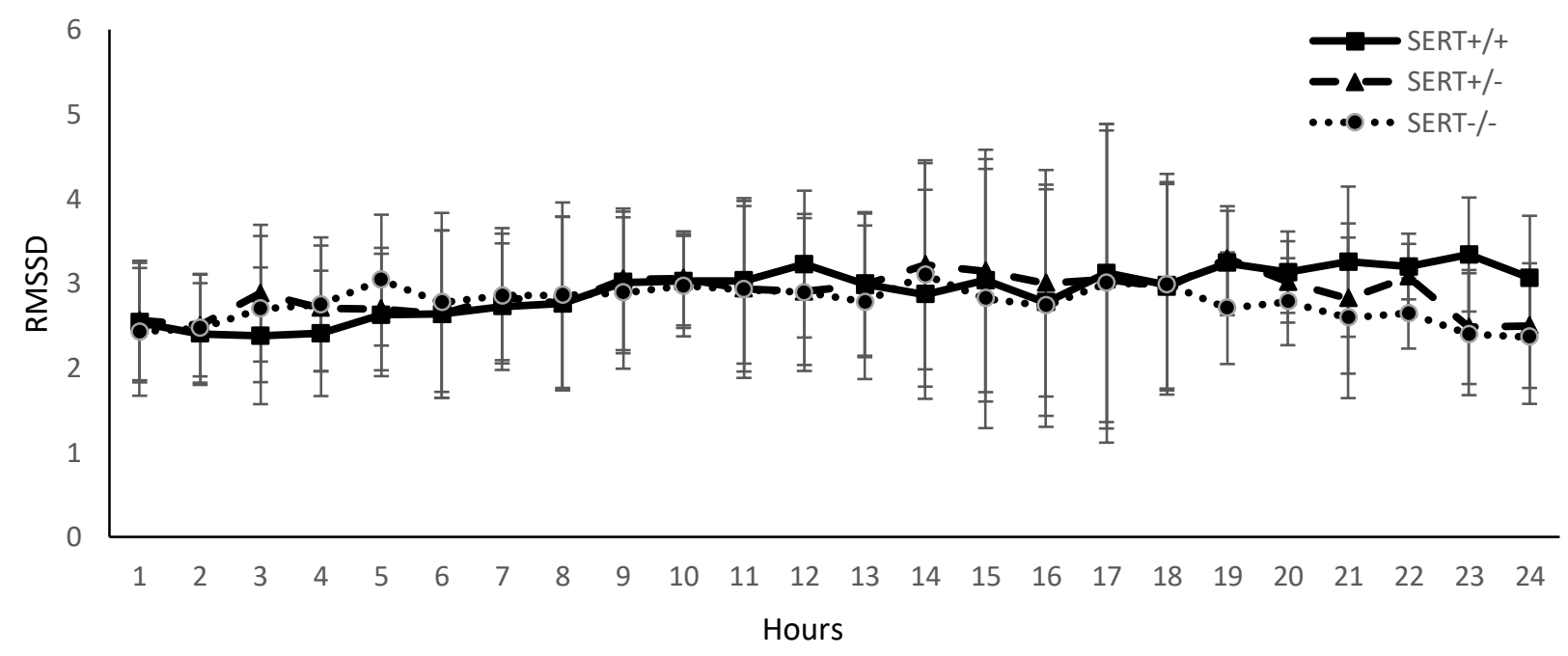

Figure 2. $\quad$ Means and \pm SEM for the average RMSSD for each hour over 24 hours. Hours (starting at $12 \mathrm{pm}$ ) are presented along the $x$ axis, while RMSSD value is depicted by the $y$ axis. $\mathrm{SERT}^{+/+}$are represented by the solid line, $\mathrm{SERT}^{+/-}$are represented by the dashed line, while the dotted line indicates the SERT ${ }^{-/}$group. No significant main effects of interactions were observed. 
Figure 3. Quarterly averaged RMSSD

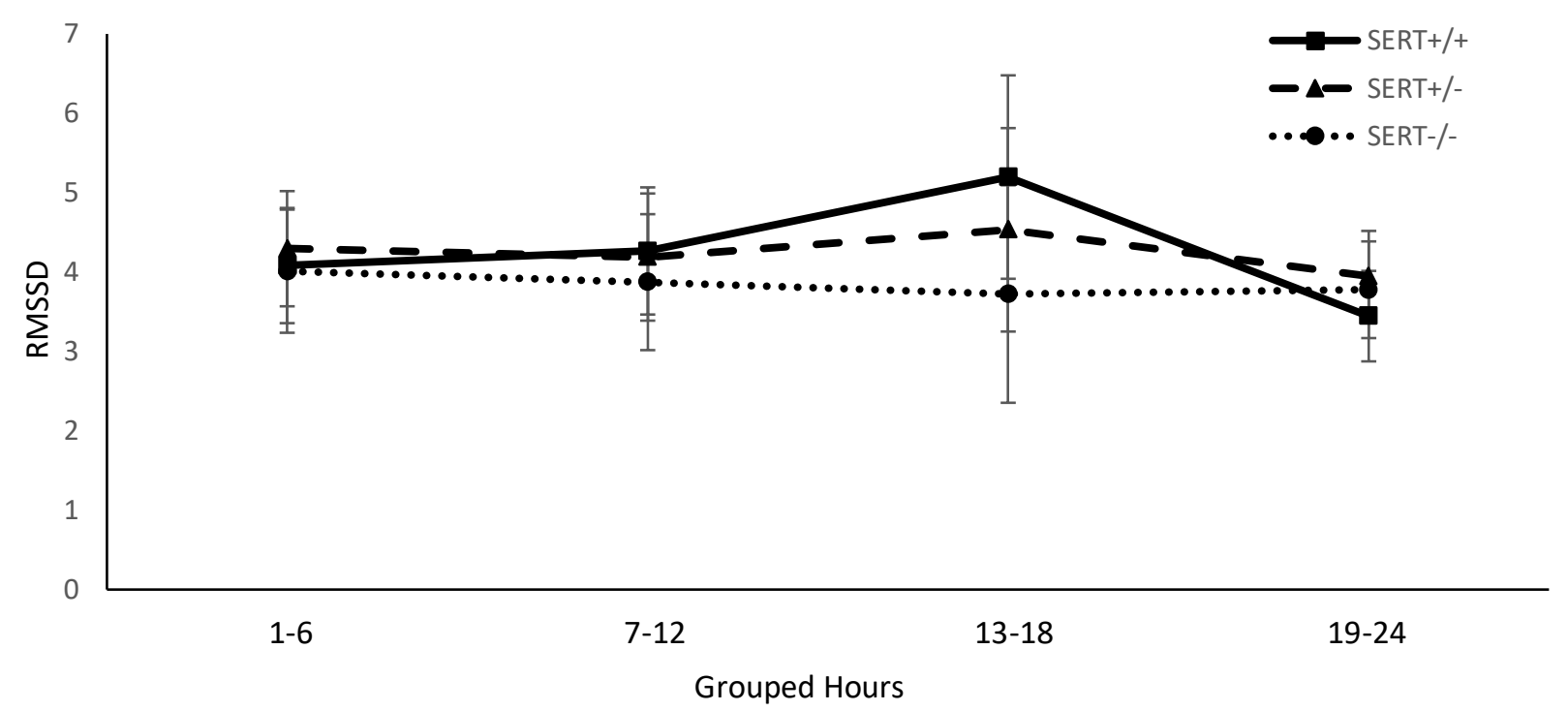

Figure 3. Means and \pm SEM for the average RMSSD for each hour over 24 hours were combined into 6 hour buckets (hours 1-6, 7-12, 13-18, \& 19-24). Quarterly buckets are presented along the $x$ axis, while RMSSD value is depicted by the $y$ axis. SERT ${ }^{+/+}$are represented by the solid line, SERT ${ }^{+/-}$are represented by the dashed line, while the dotted line indicates the $\mathrm{SERT}^{-/}$group. No significant main effects of interactions were observed.

\section{High Frequency percentage:}

Similarly to the RMSSD analyses, the means and standard deviations were computed for the average hourly HF percentage score in the $\mathrm{SERT}^{+/+}, \mathrm{SERT}^{+/-}$, and $\mathrm{SERT}^{-/-}$groups (Appendix 9). A mixed ANOVA was conducted on these data with time (Hours 1-24) as the repeated measure, and genotype $\left(\mathrm{SERT}^{+/+}, \mathrm{SERT}^{+/}, \mathrm{SERT}^{-/}\right)$as the between groups measure to examining the effect of genotype and time of day on HF percentage (Figure 4). The Greenhouse-Geisser correction was adopted due to the violation of sphericity. No significant main effects, nor interactions were observed, indicating that the effect of time and genotype have no effect on HF percentage. Following the same method as used previously, four blocks consisting of six hours each (hours 16, 7-12, 13-18, and 19-24) were produced and subjected to a mixed ANOVA, however, this simplification produced no significant effects or interactions (Figure 5). 
Figure 4. High Frequency Percentage

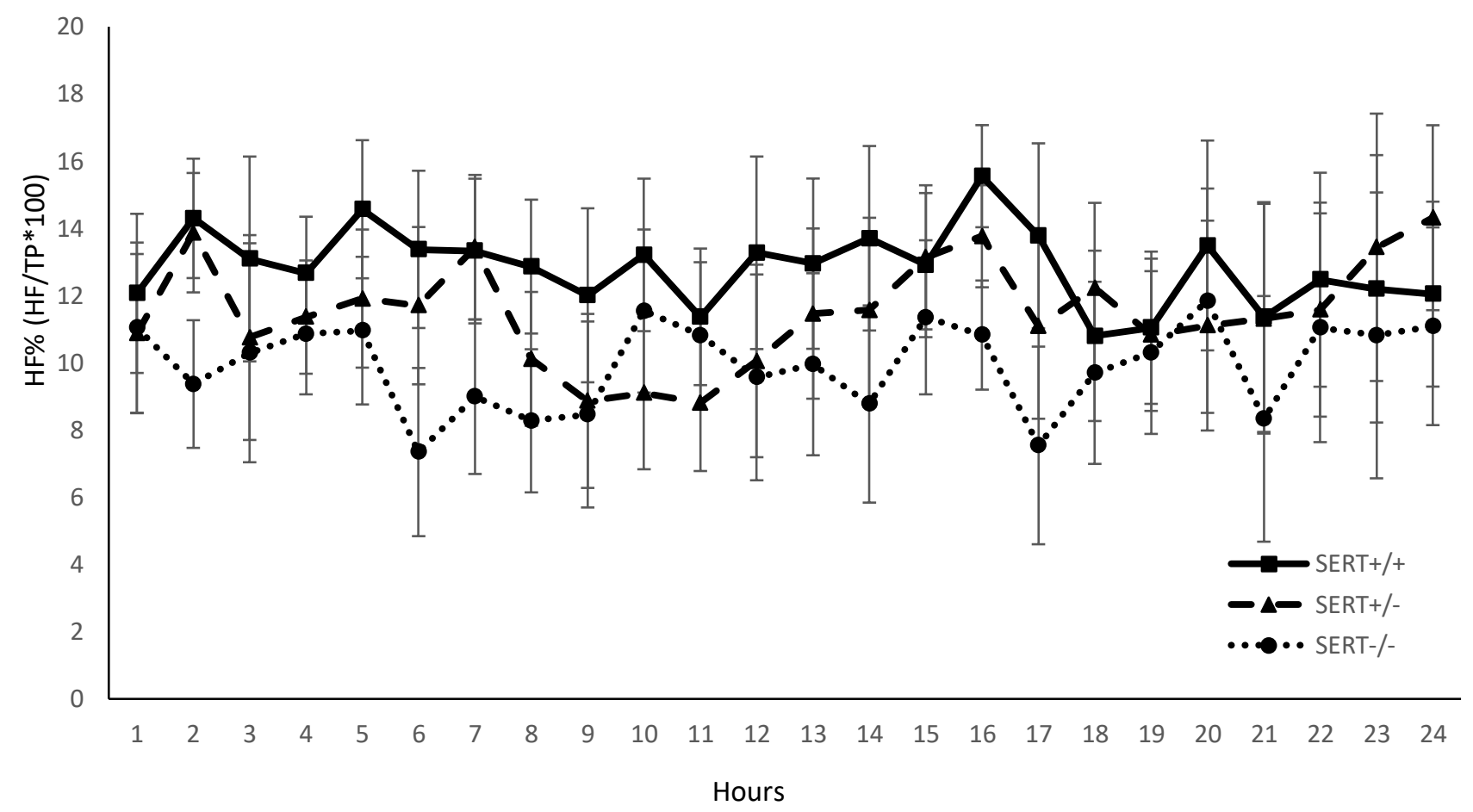

Figure 4. Means and \pm SEM for the average percentage of high frequency (HF/TP*100) for each hour over 24 hours. Hours (starting at 12pm) are presented along the $x$ axis, while the HF\% value is depicted by the $y$ axis. SERT ${ }^{+/+}$are represented by the solid line, SERT ${ }^{+/-}$are represented by the dashed line, while the dotted line indicates the SERT ${ }^{-/}$group. No significant main effects of interactions were observed. 
Figure 5. Quarterly averaged High Frequency Percentage

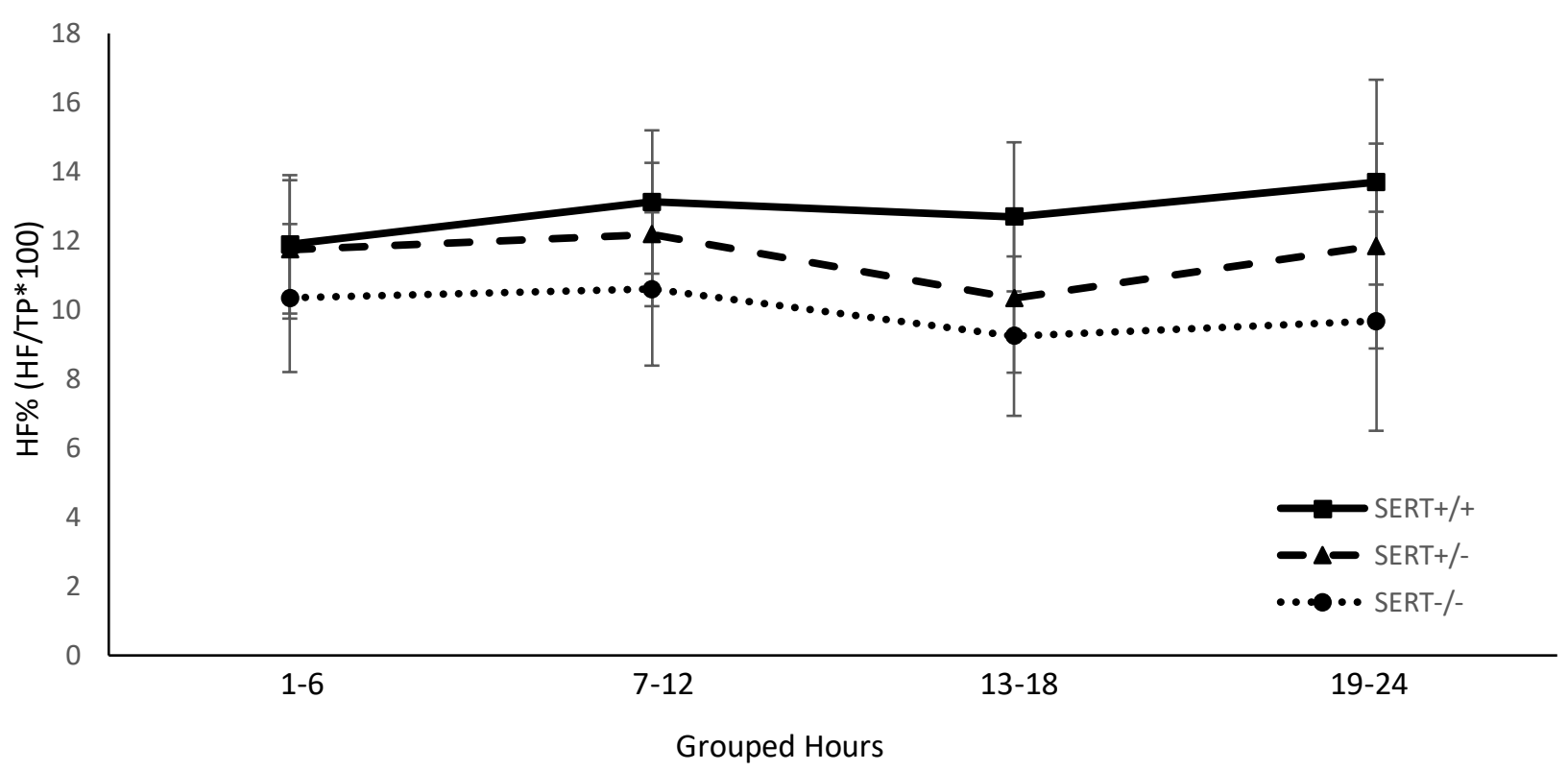

Figure 5. Means and \pm SEM for the average percentage of high frequency (HF/TP*100) for each hour over 24 hours were combined into 6 hour buckets (hours 1-6, 7-12, 13-18, \& 1924). Quarterly buckets are presented along the $x$ axis, while RMSSD value is depicted by the $y$ axis. SERT ${ }^{+/+}$are represented by the solid line, $\mathrm{SERT}^{+/}$are represented by the dashed line, while the dotted line indicates the $\mathrm{SERT}^{-/}$group. No significant main effects of interactions were observed.

Inspection of figures 2 and 4 showed that there was a relatively large variance in the RMSSD and HF values, much larger than is typically seen in the literature. To explore the potential source of this variance, we first turned our attention to the two different cohort. As the animal facility in which the experiments took place was closed and moved to a new building, the experiments had to be performed relatively quickly. This led to two important differences between the two cohorts. First of all, the second cohort was substantially younger than the first cohort. A well-documented impact of age is reduced HRV (Zhang, 2007), thus potentially resulting in an increase in the variance within the groups as a result of inter cohort variance. Secondly, and perhaps more importantly, post-operative recovery periods were reduced to the minimal ethical requirement of 5 days in cohort 2 , in order to ensure the experiments could be done before the 
lab closed. This meant that the rats were still receiving carprofen and penicillin during the actual recording (which was not the case in cohort 1).

To investigate whether these procedural differences were, at least in part, responsible for the large variance, a split analysis was performed for each cohort. Unfortunately, due to the smaller sample size, this analysis likely lacked the power to produce significant differences and thus should be regarded as representing an exploratory analysis only.

We hypothesise that if age was the driving factor underlying the variance, cohort two should show an increased RMSSD in comparison to their older counterparts. On the other hand, if the recovery caused the variance, cohort one would show an increased RMSSD value due to the additional recovery time when compared to cohort two.

Table 2.

\begin{tabular}{|c|c|c|c|c|c|c|c|c|c|c|c|c|}
\hline & \multicolumn{4}{|c|}{ SERT+/+ } & \multicolumn{4}{|c|}{ SERT+/- } & \multicolumn{4}{|c|}{ SERT-/- } \\
\hline & \multicolumn{2}{|c|}{ Cohort 1} & \multicolumn{2}{|c|}{ Cohort 2} & \multicolumn{2}{|c|}{ Cohort 1} & \multicolumn{2}{|c|}{ Cohort 2} & \multicolumn{2}{|c|}{ Cohort 1} & \multicolumn{2}{|c|}{ Cohort 2} \\
\hline & Lights On & Lights Of & ights $\mathrm{O}$ & Lights Off & Lights On & Lights Off & Lights On & Lights Off & Lights On & Lights Off & Lights On & Lights Off \\
\hline Mean & 3.15 & 3.08 & 2.65 & 2.47 & 3.24 & 2.92 & 2.74 & 2.61 & 3.01 & 2.92 & 2.86 & 2.52 \\
\hline Standard Deviation & 0.19 & 0.24 & 0.09 & 0.11 & 0.09 & 0.08 & 0.08 & 0.09 & 0.14 & 0.16 & 0.08 & 0.08 \\
\hline
\end{tabular}

A three-way ANOVA was conducted to examine the effect of cohort (1 and 2), genotype $\left(\mathrm{SERT}^{+/+}, \mathrm{SERT}^{-/+}\right.$and, $\mathrm{SERT}^{-/}$) and time of day (lights on or lights off) on RMSSD values (Figure ๑). A main effect of cohort was observed $[F(1,540)=22.86, p<.001]$, with table 2 indicating that cohort one had significantly greater RMSSD than cohort two. Furthermore, a main effect of time of day was found $[F(1,540)=4.80, p=.030]$, with table 2 indicating an intact circadian rhythm with higher RMSSD during the lights on period. Since no interactions were observed these cohort and time of day effects occurred independent of genotype (although, as mentioned above, this may in part be due to a lack of statistical power). 
Figure 6. RMSSD; Groups split by genotype, cohort, and time of day

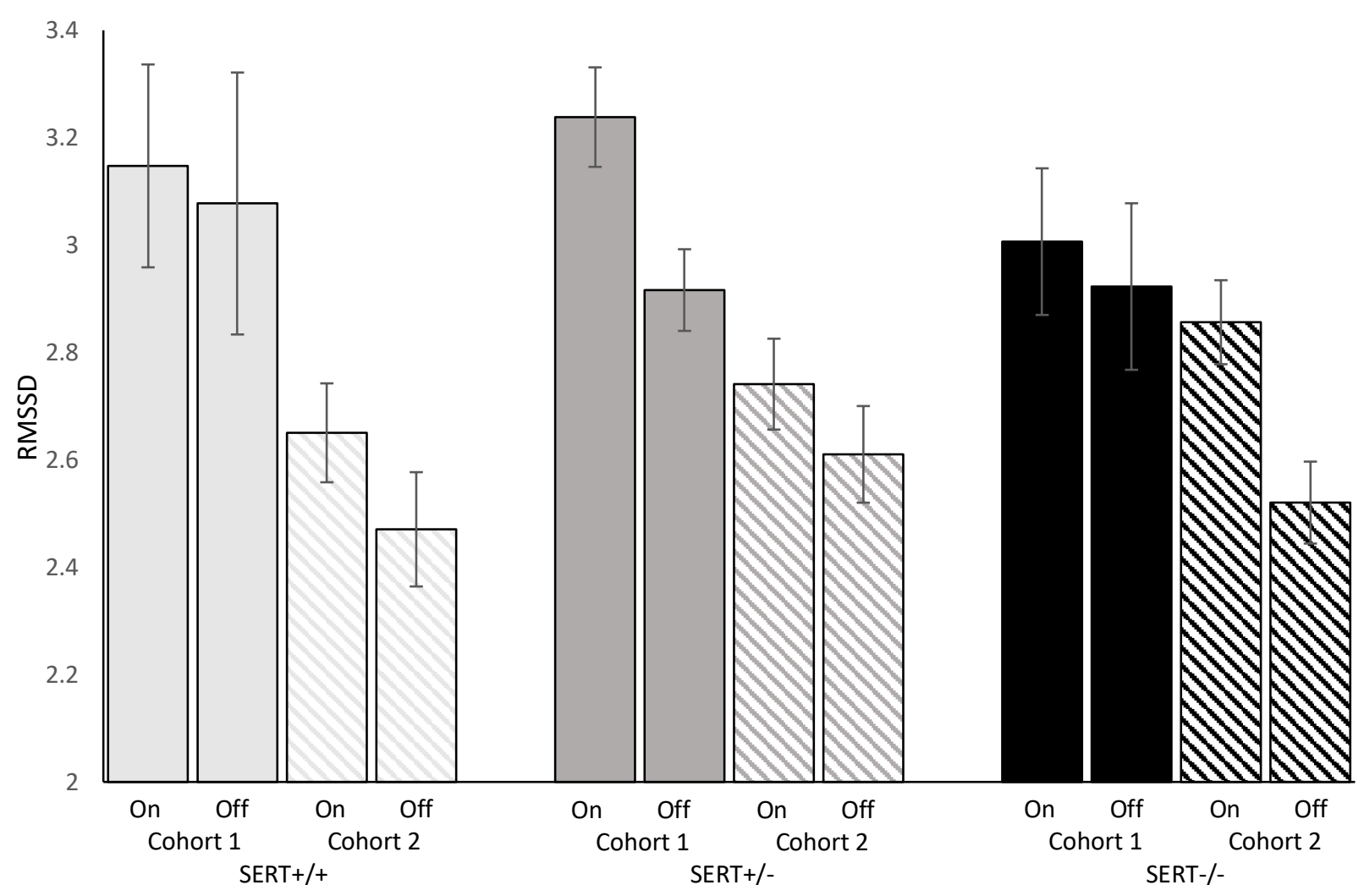

Figure 6. Means and \pm SEM for RMSSD ( $y$ axis), split by genotype, cohort, and light cycle ( $x$ axis). Main effects of cohort $(p<.001)$, and light cycle $(p=.03)$, were observed. No further interaction was observed.

Given the clear cohort effect on RMSSD, a similar analysis was performed for HF percentage. (Figure 7). A main effect of cohort was again observed $[F(1,540)=45.65 \mathrm{p}<.001]$, with table 3 showing a substantially lower percentage of HF percentages in the second cohort. We did not find a main effects of time of day, nor genotype, however, a genotype by cohort interaction was observed $[\mathrm{F}(2,540)=13.04, \mathrm{p}<.001]$. To further examine the nature of this interaction, simple comparisons were computed; the significant genotype by cohort interaction was maintained, while no further interactions were uncovered. Two follow up simple one way ANOVAs were computed for each cohort to assess the effects of genotype on $\mathrm{HF}$ percentage. In both cohort one $[\mathrm{F}(2,264)$ $=5.71, \mathrm{p}=.004]$, and cohort two $[\mathrm{F}(2,288)=15.15, \mathrm{p}<.001]$ a significant genotype effect was observed. Simple comparisons within each cohort were conducted and showed that in cohort one, 
the $\mathrm{SERT}^{+/+}$group significantly differed from the SERT ${ }^{+/-}$group $(\mathrm{p}<.001)$, while marginally differing from the SERT ${ }^{-/}$group $(\mathrm{p}=.051)$. Furthermore, in cohort two, the SERT ${ }^{+/}$group significantly differed from both SERT ${ }^{+/+}(\mathrm{p}=.004)$ and $\mathrm{SERT}^{-/-}$groups $(\mathrm{p}=.002)$, while no further differences reached significance. These data indicate that, as expected, the difference between the genotypes were substantially influenced by the recovery time.

Table 3.

\begin{tabular}{|c|c|c|c|c|c|c|c|c|c|c|c|c|}
\hline & \multicolumn{4}{|c|}{ SERT+/+ } & \multicolumn{4}{|c|}{ SERT+/- } & \multicolumn{4}{|c|}{ SERT-/- } \\
\hline & \multicolumn{2}{|c|}{ Cohort 1} & \multicolumn{2}{|c|}{ Cohort 2} & \multicolumn{2}{|c|}{ Cohort 1} & \multicolumn{2}{|c|}{ Cohort 2} & \multicolumn{2}{|c|}{ Cohort 1} & \multicolumn{2}{|c|}{ Cohort 2} \\
\hline & Lights On & Lights Off & Lights On & n Lights Off & Lights On & Lights Off & Lights On & Lights Off & Lights On & Lights Off & Lights On & Lights Off \\
\hline Mean & 15.79 & 15.18 & 8.09 & 8.82 & 11.10 & 12.16 & 11.42 & 11.44 & 12.00 & 14.59 & 9.09 & 8.17 \\
\hline Standard Deviation & 1.32 & 1.52 & 0.37 & 0.61 & 0.56 & 0.74 & 0.75 & 0.83 & 1.12 & 1.52 & 0.49 & 0.42 \\
\hline
\end{tabular}

Figure 7. High frequency percentage

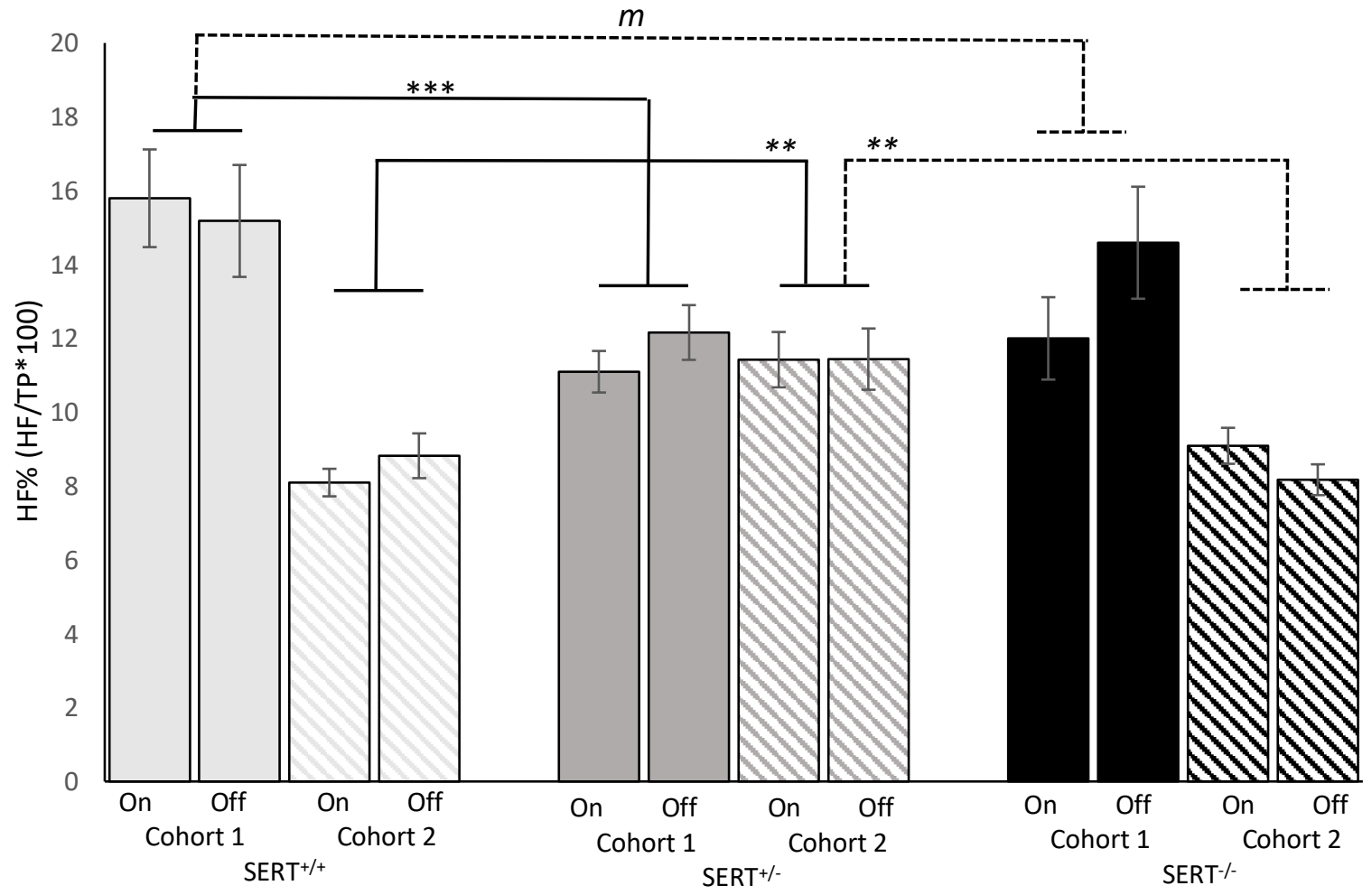

Figure 7. Means and \pm SEM for the average percentage of high frequency (HF/TP*100) ( $y$ axis), split by genotype, cohort, and light cycle ( $x$ axis). A genotype x cohort interaction was observed $(p<.001)$. Follow-up analyses revealed that in cohort 2 , SERT ${ }^{+/}$animals displaced higher $\mathrm{HF} \%$ when compared to $\mathrm{SERT}^{+/+}(p<.004)$, and SERT ${ }^{-/}$groups $(p<.002)$. Furthermore, within cohort one, SERT ${ }^{+/+}$animals demonstrated a greater $\mathrm{HF} \%$ when compared to SERT ${ }^{+/-}(p<.001)$ and SERT ${ }^{-/-}$animals $(p=.051)$.

$m \quad .05<p>.09, * * \quad p<.005, * * * \quad p<.005$ 


\section{Cardiac morphology}

While we are unable to conclusively state that the genetic reduction of serotonin transporter expression results in significant alterations in HRV, the data suggest this trend may be present, particularly in cohort 1. Abnormal HRV is likely to be accompanied by morphological alterations of the heart as well. To get a first impression of such possible morphological differences, we measured the heart weight of the animals in the different genotype. To account for the possible age differences in the cohort, we corrected for total body weight. It should be noted that body weight has been shown to not differ between $\mathrm{SERT}^{+/+}$and $\mathrm{SERT}^{-/-}$animals of similar age. Increased HW:BW has been shown in prior animal models of depression and anxiety (Carnevali \& Sgoifo, 2014), thus it is hypothesised that $\mathrm{SERT}^{-/-}$rats had a significantly increased HW:BW in comparison to $\mathrm{SERT}^{+/+}$, with $\mathrm{SERT}^{+/-}$falling between these groups resulting in a gene dose response (refer to Appendix 10 for extraction procedure).

\section{Results}

A one-way ANOVA was conducted (Figure 8) to assess potential genotype differences. Levene's test of homogeneity revealed no differences in variance between groups. A marginally significant effect of genotype was observed $(F(2,23)=2.90, p=.077$, which while not significant at the .05 level, warranted further examination into the underlying differences. A post-hoc LSD analysis showed a significant difference between SERT ${ }^{+/+}$and SERT ${ }^{-/-}(\mathrm{p}=.035)$, and marginal differences between SERT ${ }^{+/}$and SERT ${ }^{-/-}(\mathrm{p}=.064)$. It should be noted that these effects would not withstand a correction for multiple testing. While the significance is marginal at best, these results suggest a trend whereby the SERT ${ }^{-/}$genotype showed an increased HW:BW. 
Figure 8 . Heart to body weight ratio

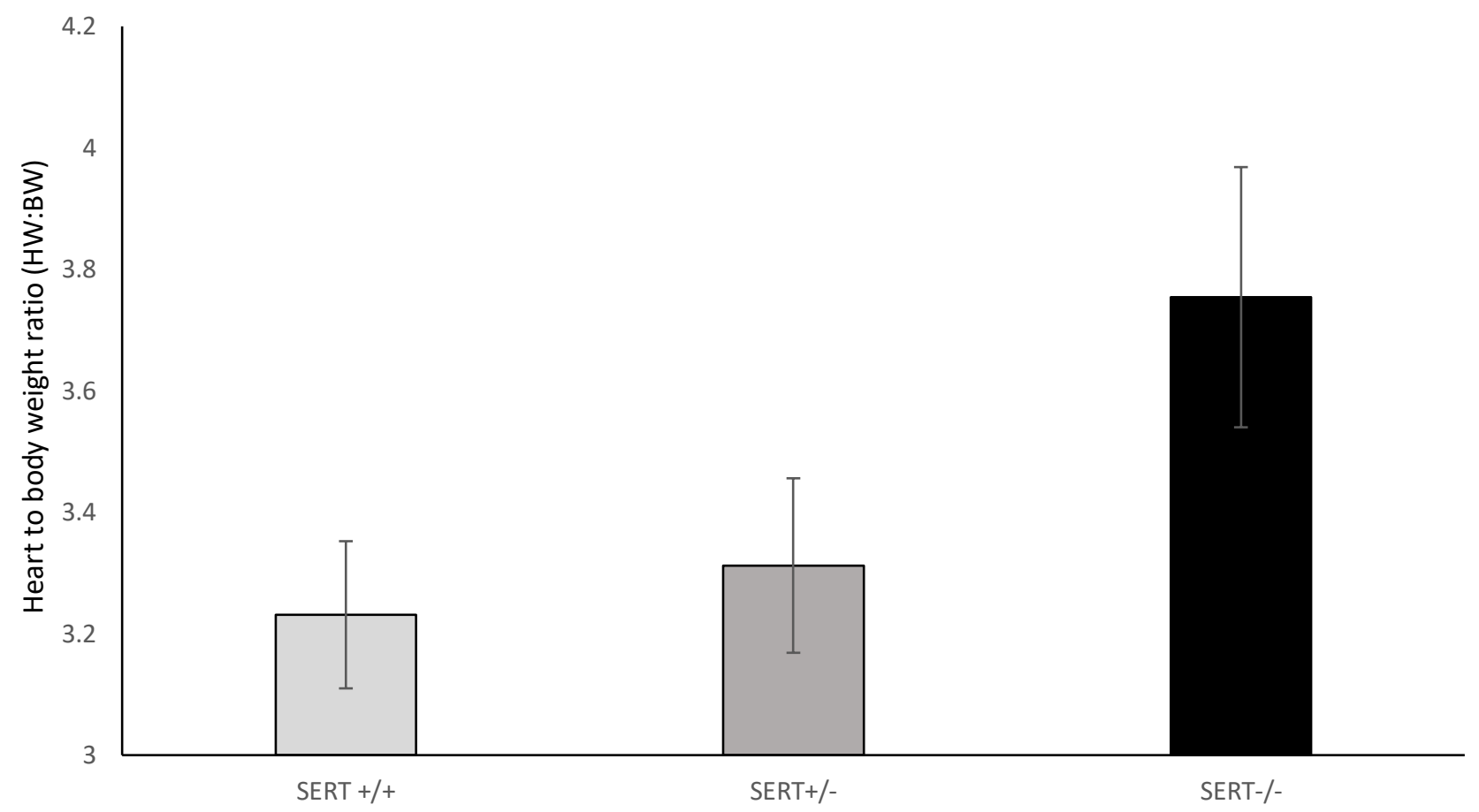

Figure 8. Means and \pm SEM for the heart to body ratio (HW:BW) between genotypes. HW:BW values presented along the $y$ axis, and genotypes along the $x$ axis. The SERT ${ }^{-/-}$ group significantly differed from the SERT ${ }^{+/+}$group $(p=.064)$, while only marginally differed from the SERT ${ }^{+/}$group $(p=.064)$, neither of which would withstand correction for multiple testing. No other differences were observed.

\section{Discussion}

Given that HRV changes have been reported in major depression, as well as in many other psychiatric disorders, combined with the fact that it can be assesses in animals and humans with virtually identical methods, makes HRV a very interesting candidate for assessing novel animal models of psychiatric disorders (Kidwell \& Ellenbroek, 2018a). Furthermore, normalisation of HRV through novel pharmacological interventions in animal models can be used to assess the therapeutic window in humans at a much earlier stage of clinical development than assessing the improvement of psychiatric symptoms. One limiting factor in the analysis of HRV is the plethora of different methods of assessing HRV. As mentioned in the introduction, many linear and non- 
linear methods have been developed. The present study examined a variety of HRV parameters in $\mathrm{SERT}^{+/+}, \mathrm{SERT}^{+/-}$, and $\mathrm{SERT}^{-/-}$animals to determine whether the genetic reduction in serotonin transporter expression in rats would produce similarities to depressed humans, thus providing a novel animal model of depression with high translational validity. Given that in such patients, reductions have been described particularly in RMSSD and HF, we focussed predominantly of these two. We hypothesized a gene dose related reduction in HF percentage and RMSSD (van der Kooy et al., 2006). While there appeared to be a trend in support of this hypothesis, the data showed an unexpected large variance, resulting in a failure to find significant differences.

Due to this, the objective of the data analysis became focussed on determining the source of the variance. Two hypotheses were tested based on the fact that the second cohort of animals had to be tested more quickly as the animal lab was about to be closed. First it was hypothesised that if the age differences was responsible for the large variance, cohort one should have a lower RMSSD as they were older (Zhang, 2007). Secondly, if the short post-operative period (and the continued used of penicillin and carprofen) was responsible, the first cohort should have higher RMSSD, (Maesen, Nijs, Maessen, Allessie, \& Schotten, 2011). The analysis of these data revealed a greater RMSSD value in cohort one, thus supporting the second hypothesis.

Due to the time constraints previously mentioned, the second cohort were receiving postoperative antibiotics and pain relief during the time of testing, whereas the first cohort received an additional two days of recovery following the final postoperative infusion. An unexpected result of these medications could be hyperkalaemia, an abnormally high serum level of potassium. Both carprofen, among other nonsteroidal anti-inflammatory drugs (NSAIDs), and penicillin have been shown to increase the risk of hyperkalaemia (Perazella, 2000; Thiele \& Rehman, 2008) which has been demonstrated to significantly impact the morphology of a standard P QRS T wave (Ettinger, Regan, \& Oldewurtel, 1974). In particular, increased potassium results in a blunted and elongated $\mathrm{p}$ wave through modulation of the sinoatrial node depolarisation. To explore this possibility, the 
P QRS T wave morphology was examined between high and low HRV animals. An average P QRS T of 80 cycles was randomly selected, during the active and the rest period for the two animals displaying the highest, and lowest HRV (figure 9). To account for individual differences in signal strength, the ratio between the height of the p-wave, and r-wave were computed. The P:R ratio was shown to be significantly reduced in the low HRV animals, both of which were from the second cohort (appendix 11).

Figure 9. Representative average P QRS T waves from each cohort

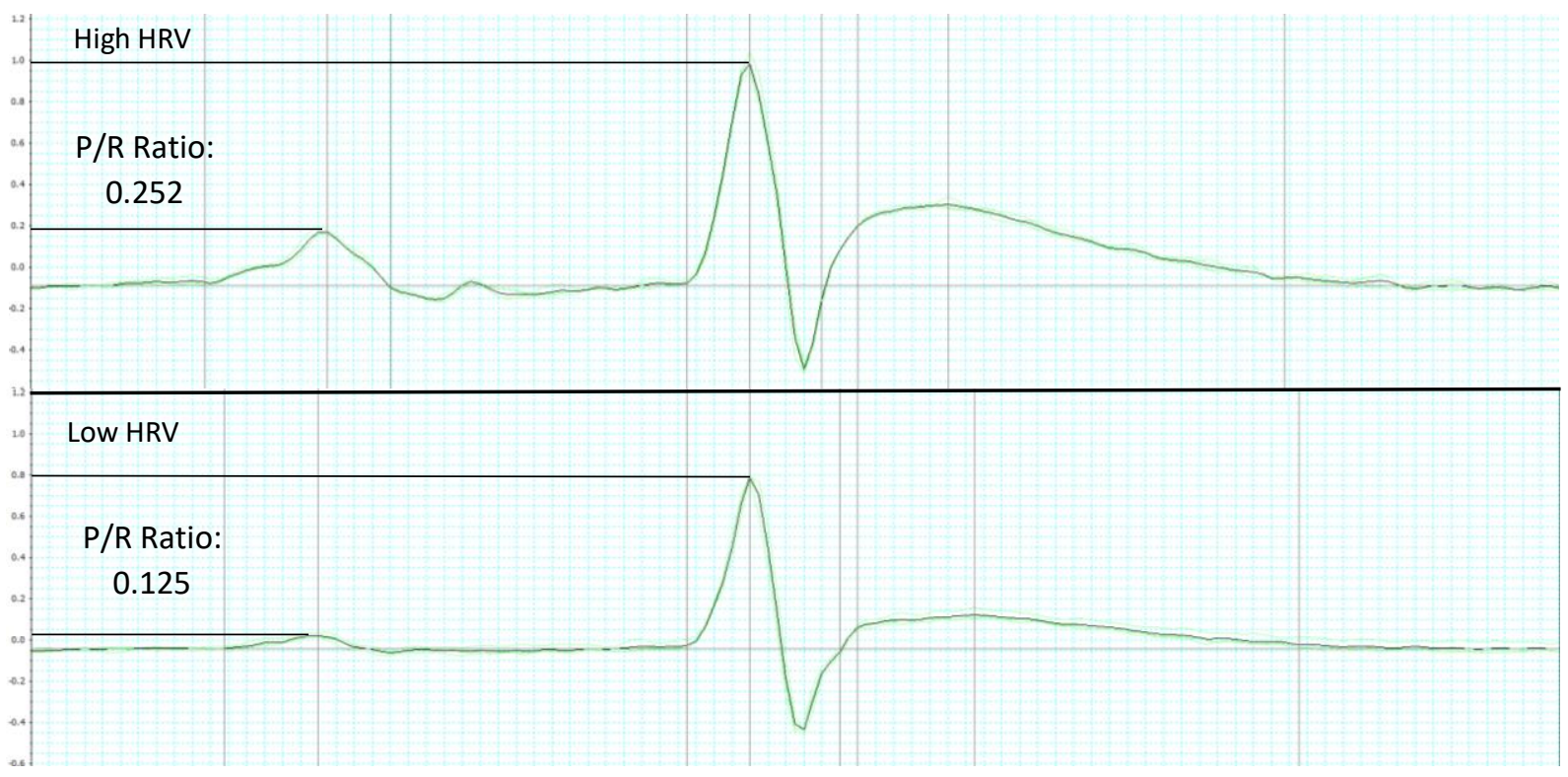

While no study has directly examined the impact of hyperkalaemia on HRV, this morphological change strongly suggests the existence of such an association. Furthermore, an abnormal p-value is most likely to directly impact HF components of HRV, as HF is generally considered a measure of respiratory sinus arrhythmia, the modulation of the sinoatrial node by respiration (Yasuma \& Hayano, 2004). While we were unable to directly examine the serum potassium concentration, as this effect was only detected following euthanasia, the examination of the HF percentage data strongly suggests a significant impact of potassium to be present. To highlight this, the split analysis of HF percentage showed no effect of circadian pattern, a highly robust effect observed even in the RMSSD of these animals. Furthermore, a genotype by cohort 
interaction was observed, whereby differences between $\mathrm{SERT}^{+/+}$and $\mathrm{SERT}^{-/}$animals began to emerge within cohort one, further emphasising the normalisation of HRV following increased recovery time. The lack of such a genotype effect in cohort 2 may be due to a floor effect, as HF and RMSSD were already very low in this cohort due to the reasons mentioned above. While these data should not be considered conclusive due to the low sample size requiring the violation of statistical assumptions to increase power, they should still be considered to suggest that the genetic reduction of serotonin transporter expression may produce reduction in $\mathrm{HRV}$, improving the translational validity of the serotonin transporter knockout rat as a model of depressive disorders, warranting further investigation.

Finally, to further examine the likely association between the reduced serotonin expression and the cardiovascular system, cardiac morphology was examined through the computation of HW:BW. It was hypothesised that $\mathrm{SERT}^{-/}$animals will be observed to have an increased HW:BW, which while not reaching significance, suggests a trend in support of this hypothesis. There data provide additional support to the suggested association between cardiovascular abnormalities and alterations of the serotonergic system frequently reported in humans. For example, individuals with autism spectrum disorder, particularly those exposed to valproic acid during foetal development, show abnormal heart valve morphology (Jentink et al., 2010), as well as reduced HRV (Ellenbroek \& Sengul, 2017). While having multiple effects, Valproic acid has been shown to significantly impact the serotonergic system, whereby foetal serum concentrations are significantly increased; the most frequently reported association between gestational exposure and the development of autism (Narita et al., 2002). Given the similar serotoninergic impact of gestational valproic acid, and the genetic reduction in serotonin transporter expression, it is unsurprising that abnormal heart valve morphology has been reported in SERT knock-in mice (transporter expression reduction during gestation, thus comparable to the SERT knockout line during this period) (Pavone et al., 2008). This association is further demonstrated through foetal exposure to SSRIs producing cardiac malformations (Noorlander et al., 2008), and a reduction in 
left ventricular weight being observed in SERT ${ }^{-/-}$rats (J. Homberg et al., 2006). While the precise mechanisms underlying this association at yet to be fully elucidated, serotonin has been demonstrated to play a significant role in cardiovascular development. For example, Nebigil and colleagues. (2000) developed an animal model in which a genetic ablation of the $5-\mathrm{HT}^{2 \mathrm{~B}}$ receptor subtype results in embryonic and early infant death due to heart malfunctions. Due to the associations between HRV, cardiovascular morphology, and the serotonergic system, the examination of the cardiovascular morphology in $\mathrm{SERT}^{-/-}$and $\mathrm{SERT}^{+/-}$may further elucidate the association between the association between the 5-HTTLPR polymorphism, depressive disorders, and HRV abnormalities. It should be noted that the data showed an inability to reach significance, likely resulting from the tissue extraction and preparation, whereby hearts were extracted, then rapidly snap-frozen for use in later experiments. Due to this, residual blood remained in the ventricles and atria, likely contributing to the variance observed.

Due to the lack of clear significance when examining the effects of the genetic reduction in the expression of the serotonin transporter on the cardiovascular system due to the limitations described, clear conclusions are difficult to make. Due to this, the reduced HF percentage and RMSSD parameters observed in the SERT ${ }^{-/}$rats in the first cohort will be discussed despite the violation of statistical assumptions. These data suggest a potential reduction in HRV parameters as a result of the reduction in serotonin transporter expression. While as demonstrated with potassium, numerous factors have been shown to significantly impact HRV scores in humans, including diet and exercise (H. A. Young et al., 2017), prior studies have shown that SERT ${ }^{-/}$rats have normal locomotor activity in these animals (Lizarraga et al., 2014; J. Olivier et al., 2008). Furthermore, serotonin transporter knockout animals typically do not significantly differ in body weight, nor in food consumption (Lira et al., 2003).

While no direct effects are present, it has been demonstrated that activation of the $5-\mathrm{HT}^{1 \mathrm{~A}}$ receptor inhibits the release of the inhibitory neurotransmitter, GABA (Kishimoto, Koyama, \& 
Akaike, 2001). This inhibition of GABA in turn results in a reduction of vagal tone. Although at this point, it is speculative to discuss the underlying mechanism, it has been well established that 5-HT ${ }^{1 \mathrm{~A}}$ receptor activation enhances vagal tone (REFS). Given the SERT ${ }^{-/-}$rats have substantially reduced $5-\mathrm{HT}^{1 \mathrm{~A}}$ activation, vagal tone is likely reduced. as well. (J. D. Olivier et al., 2008). If the current trends can be statistically confirmed in replication studiers, it would strongly suggest the association between reduced serotonin transporter expression and cardiovascular abnormalities... Moreover, it would substantially add to the relevance of the $\mathrm{SERT}^{-/}$as a genetic model for depression, as a reduction in vagal tone and HRV has consistently been reported in human studies in depression (Kidwell \& Ellenbroek). In line with this suggestion, one of the most direct links between HRV and depression comes from studies using vagus nerve stimulation.

Given the invasive nature of the procedure, VNS has so far only been used in treatmentresistant cases of MDD. Sackeim et al. (2001) piloted this technique in 60 treatment-resistant MDD patients, to establish predictors of outcome and side effects, and to determine the response rate to this novel technique. The 10 -week response rate ranged from 30.5 to $37.3 \%$ improvement, depending on which depression scale was used for the assessments, with relatively few side effects. On the basis of this finding, VNS was proposed as an effective technique to overcome mild to moderate treatment-resistant MDD. Marangell et al. (2002) conducted a 1-year follow-up of these patients to examine the chronic efficacy of VNS and reported that response rates were maintained, while remission rates had significantly increased. Finally, a 2-year follow-up was conducted by Nahas et al. (2005) who reported sustained response and remission rates, from which they suggested VNS as an effective treatment for resistant MDD or chronic recurrent MDD.

The present study initially sought to examine the impact of the genetic reduction of serotonin transporter expression on parameters of HRV. This was initially intended to improve the translational validity of the serotonin knockout rat as a model of depressive disorders, however, due to uncontrollable time constraints, the effects were significantly impacted by a high degree of 
variance. The increase in potassium following standard post-operative protocols was determined to underlie this variance, and once controlled for, genotype differences emerged. Furthermore, HW:BW was computed to elucidate the mechanisms underlying the association between serotonin and cardiovascular alterations. These data showed a marginally significant increase in HW:BW in $\mathrm{SERT}^{-/-}$animals. While caution should be taken when interpreting these results, these data suggest cardiovascular pathology in $\mathrm{SERT}^{-/}$rats, similar humans with psychiatric illness. This in turn suggests the association between $\mathrm{SERT}^{-/-}$rats and depressed humans extends beyond behavioural associations, improving the translational validity of the model. These data should be replicated following the recommendations listed to further understand the association between HRV and depressive disorders. 


\title{
CHAPTER FIVE
}

\author{
Experiment Four
}

The Impact of the Serotonin Transporter Knockout on Early

Neurogenesis 
Given the behavioural abnormalities associated with the 5-HTTLPR genetic variation, a large variety of psychopathologies coexist (Kenna et al., 2012). However, the underlying neurobiological alterations are yet to be fully elucidated. Of particular interest in this respect is the so-called serotonin paradox. In its simplest form, the serotonin paradox is based on the fact that on the one hand the most common medications given to treat anxiety and depressive disorders are SSRIs which selectively inhibit serotonin transporter function, thus increasing extracellular serotonin (Nutt et al., 1999). On the other hand, as shown in the literature and previous chapters, a genetic mutation which similarly impacts the serotonin transporter produces the opposite effects, i.e. an increase in anxiety and depressive symptoms, in both animals and humans. (see figure 1). The major difference between that two situations is, of course, that SSRIs are typically administered during adulthood, while 5-HTTLPR mutations create alterations in serotonin transporter functionality during development, thus likely impacts all stages of neurodevelopment. This suggestion is supported by perinatal exposure to SSRIs producing effects comparable with the SERT knockout (Oberlander, Gingrich, \& Ansorge, 2009). Serotonin plays a diverse, and very important role in all stages of neurodevelopment, affecting particularly prenatal and early postnatal development (Kepser \& Homberg, 2015). Details of the involvement of serotonin across an array of neurodevelopmental processes will be briefly described below.

Figure 1. Visual depiction of the serotonin paradox
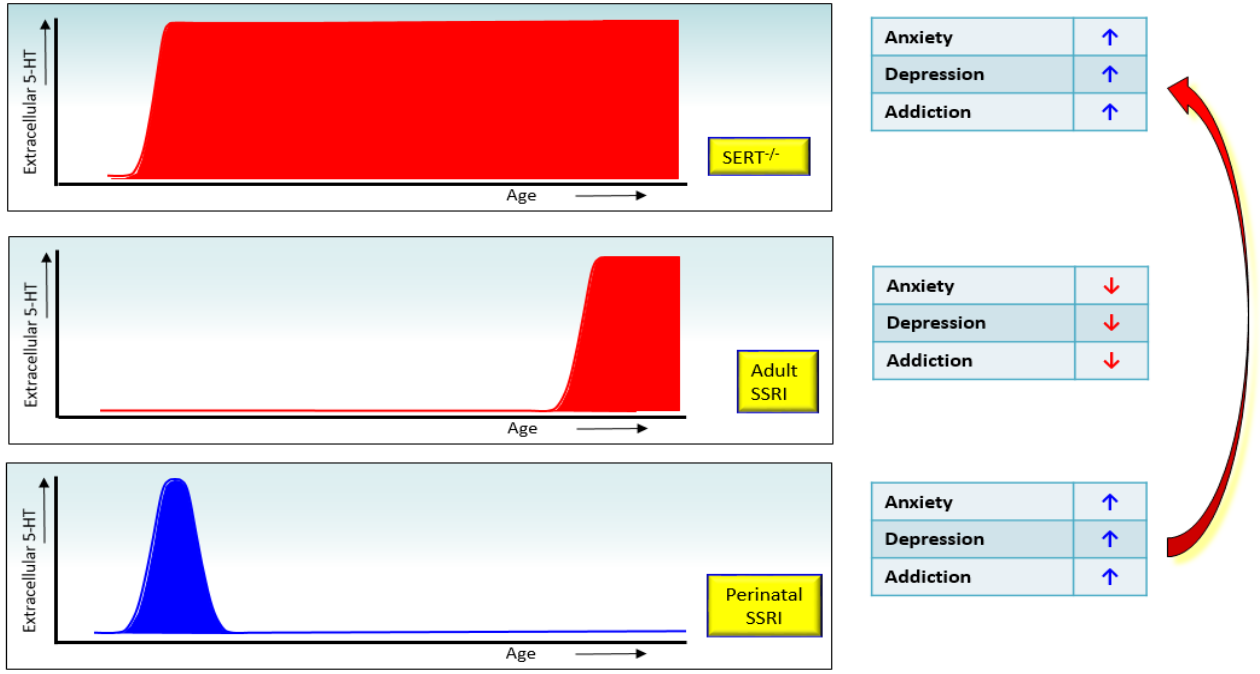
Serotonin plays a crucial role in many aspects of neurodevelopment, thus abnormalities in extracellular serotonin during development can produce significant alterations to the natural development of a foetus and infant. For example, serotonin modulates axon path finding through the protein, netrin-1, which when exposed to excessive serotonin reverses its function, repelling extending axons (Bonnin, Torii, Wang, Rakic, \& Levitt, 2007). Other notable influences of excess serotonin during cerebral cortex development include protection against normal neuron culling, increased synaptogenesis, angiogenesis, gliogenesis, and neurogenesis (Vitalis \& Parnavelas, 2003). While the protective effects being detrimental may appear paradoxical, the developing brain is highly sensitive, thus alterations in the natural homeostasis will produce significant downstream effects. Homberg, Schubert, and Gaspar (2010) reviewed the literature surrounding the impact of increased serotonin during early neurodevelopment from both animal and human studies. The authors identified associations between the early increased serotonin concentrations and increased anxiety and depressive symptoms. While these studies were primarily from animal research, the fundamental impacts of serotonin on neurodevelopment are translatable to human development. Given the broad impact of increased serotonin on neurodevelopment, it is unsurprising that there exists a strong association between the 5-HTTLPR polymorphism and a wide range of psychiatric illnesses (Caspi \& Moffitt, 2006).

Of particular interest is the impact of serotonin on neurogenesis which refers to the development of new neurons. Neurogenesis is a crucial element in the developing brain with neurogenesis rates maximising during late gestation then reducing with age (Kuhn, DickinsonAnson, \& Gage, 1996). Abnormal neurogenesis has been implicated in a number of psychiatric disorders including anxiety, autism and schizophrenia (Kheirbek, Klemenhagen, Sahay, \& Hen, 2012; Reif, Schmitt, Fritzen, \& Lesch, 2007; Revest et al., 2009; Wegiel et al., 2010), however, most frequently within depressive disorders (Kempermann \& Kronenberg, 2003; Sahay \& Hen, 2007). 
Adult neurogenesis was first detected in humans using 5-bromo-2'-deoxyuridine (BrdU) by Eriksson et al. (1998), to discover the development of new cells in the dentate gyrus. Since this discovery, the results have been replicated with mixed success in human studies, potentially due to a variety of procedural differences (Bhardwaj et al., 2006; Sorrells et al., 2018). Despite this controversy, the results indicating that neurogenesis occurs throughout development and adulthood in non-human mammals remains relatively consistent (Kuhn et al., 1996; Ming \& Song, 2005). The involvement of serotonin in neurogenesis has been relatively well studied, with serotonin depletions reducing neurogenesis within the dentate gyrus (Brezun \& Daszuta, 1999). Further, due to the delayed response to SSRI treatment aligning closely with the time course for new cells to develop and become integrated into the dentate gyral network, this has been posited as a potential mechanism underlying the effects of antidepressants (Jacobs, Van Praag, \& Gage, 2000). Thus, serotonin clearly plays a crucial role in neurodevelopment through the promotion of neurogenesis throughout development and possibly into adulthood.

Although behavioural and neurological data remains relatively consistent in regards to the impacts of abnormal serotonergic system activity, it is important to explore the impacts of serotonin on neurogenesis from a molecular and cellular perspective. While during development a number of neurons have been shown to temporarily store and release serotonin (Lebrand et al., 1996), the production and release of serotonin remains relatively specific to the serotonergic system, especially later in life (Jonnakuty \& Gragnoli, 2008). The serotonergic system originates from the raphe nuclei, a cluster of nuclei located within the brain stem. During early cortical development, afferent fibres project from the raphe nuclei into the majority of cortical regions (Wallace \& Lauder, 1983). The complexity of serotonergic effects is further explained by serotonin's self-regulatory ability, whereby serotonin can stimulate or inhibit its own expression (Gaspar et al., 2003; Lauder, 1990). The hippocampus is one region where serotonergic projections are most densely populated, thus it is one of the primary regions of interest when examining the function of neurogenesis. 
There exists 14 types of serotonin receptors, of which the majority have been implicated in neurogenesis, however, $5-\mathrm{HT}^{1 \mathrm{a}}$ receptor activation has been shown to be most strongly associated with increased neurogenesis in adulthood, while others remain less understood (Banasr \& Duman, 2007). Activation of the $5-\mathrm{HT}^{1 \mathrm{a}}$ receptor subtype has been shown to reduce the cAMP cascade, while increasing the rate of neurogenesis (Duman, Malberg, Nakagawa, \& D’Sa, 2000; Duman, Malberg, \& Thome, 1999). Further, 5-HT $\mathrm{T}^{4,6, \&} 7$ receptor subtypes have been shown to trigger the cAMP-CREB cascade, which in turn increase the expression of BDNF (Djavadian, 2004). While BDNF itself does not directly influence the rate of neurogenesis, BDNF has been shown to increase the release of serotonin (Martinowich \& Lu, 2008). This increased release of serotonin then likely stimulates the $5-\mathrm{HT}^{1 a}$ receptors, increasing adult neurogenesis, as well as the $5-\mathrm{HT}^{2 \mathrm{~b}}$ receptors, which are involved in early neurogenesis. One potential explanation behind the serotonin paradox is that due to increased extracellular serotonin during development, downregulation of the $5-\mathrm{HT}^{1 \mathrm{a}}$ receptor occurs, resulting in reduced adult neurogenesis, which has been associated with depression. In addition, higher levels of serotonin during development would lead to overstimulation of the $5-\mathrm{HT}^{2 \mathrm{~B}}$ (and the $5-\mathrm{HT}^{1 \mathrm{~A}}$ receptors) resulting in increased developmental neurogenesis.

A number of studies have examined the effects of neurogenesis through the agonism and antagonism of the $5-\mathrm{HT}^{1 \mathrm{~A}}$ receptor (Polter \& $\mathrm{Li}, 2010$ ), whereby $5-\mathrm{HT}^{1 \mathrm{~A}}$ activation produces increased neurogenesis, while the inverse occurs in blockades and $5-\mathrm{HT}^{1 \mathrm{~A}}$ knockout rodents. While some studies have indeed reported reduced neurogenesis in adulthood, these effects are not observed in SERT knockout animals (Murphy \& Lesch, 2008), while studies looking at neurogenesis in early development are lacking. To further examine the nature of the serotonin paradox, we aimed, in the present chapter to investigate whether a genetic reduction in serotonin transporter expression indeed leads to increases in neurogenesis in the developing brain. 


\section{BrdU immunohistochemistry}

Several different methodologies exist for the detection of neurogenesis, including analysis of the immunohistochemical detection of $\mathrm{Ki}-67$, (a protein that is expressed during all phases of cell division except the resting phase) and doublecortin (a protein which is expressed during active cell division and over three weeks of maturation) (Kee, Sivalingam, Boonstra, \& Wojtowicz, 2002). However, immunohistochemical detection of 5-bromo-2'-deoxyuridine (BrdU) remains the most often used technique, particularly as it has the clear advantage of being incorporated in the DNA, and remains therefore is detectable throughout the lifespan of the cell (O'Leary et al., 2018).

$\mathrm{BrdU}$ is a synthetic analogue of the pyrimidine deoxynucleoside, thymidine (Figure 2), which when administered, incorporates itself into newly synthesised DNA, replacing thymidine in the process (Taupin, 2007). The cell cycle lasts approximately 12 hours, of which the forming cell spends 4 hours in the synthesis phases. Briefly, during the synthesis phase DNA is replicated to create two identical strands. The double helix formation of the DNA is separated primarily thought the action of helicase enzymes, which is followed by the nucleotide pairing, whereby the purine adenine pairs with the pyrimidine thymine, while the purine guanine always pairs with the pyrimidine cytosine primarily through the action of DNA polymerase $\delta$. The S-phase is later ceased by $\mathrm{p} 16$ protein (cyclin-dependent kinase inhibitor $2 \mathrm{~A})$. This process in turn produces two identical DNA strands to be separated into their own cells through the later process of mitosis. BrdU competes with thymidine, and with the correct concentration is able to near completely replace the integration of thymidine with adenine (see Figure 3). Following the incorporation into the newly formed cell, BrdU can be labelled for detection using immunohistochemistry techniques to examine neurogenesis, cell proliferation, cell migration, and cell survival. The half-life of BrdU is 11 minutes, and while BrdU is present, all cells passing through the $\mathrm{S}$ phase will be labelled, however to avoid cell migration to examine accurate, regionally specific neurogenesis, the animal should be euthanized within two hours of BrdU administration. 
Figure 2. Molecular similarities between BrdU and Thymidine

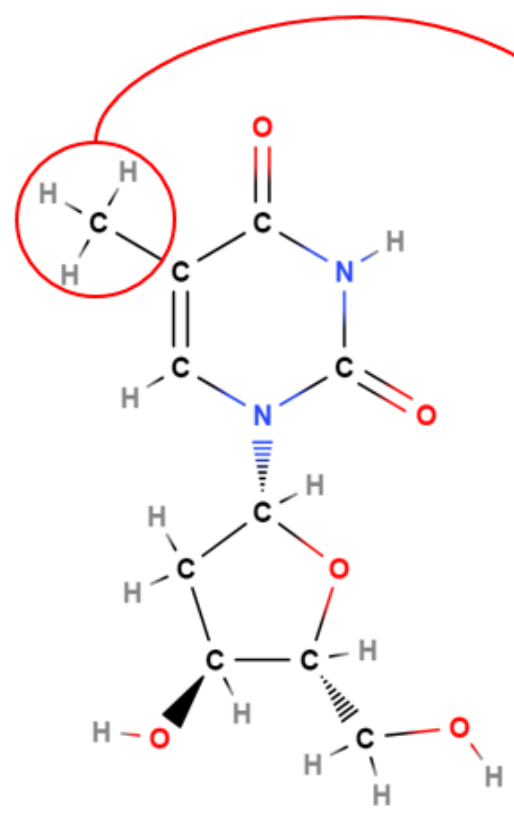

Thymidine
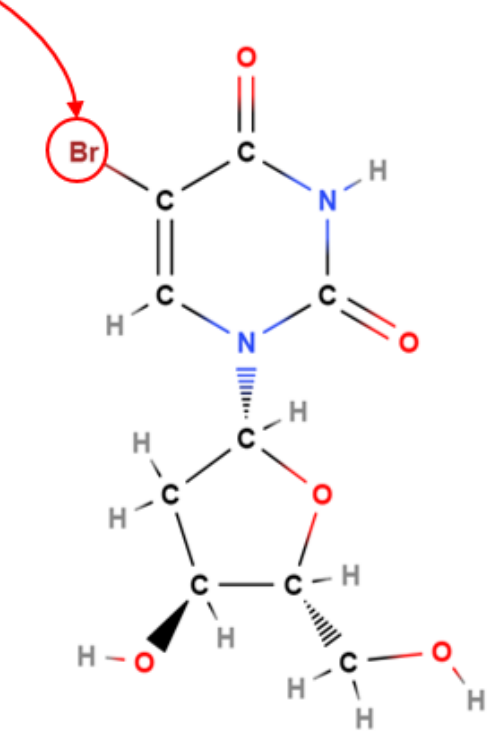

5-bromo-2'-deoxyuridine (BrdU)

Figure 3. The BrdU incorporation process which allows antibody labelling

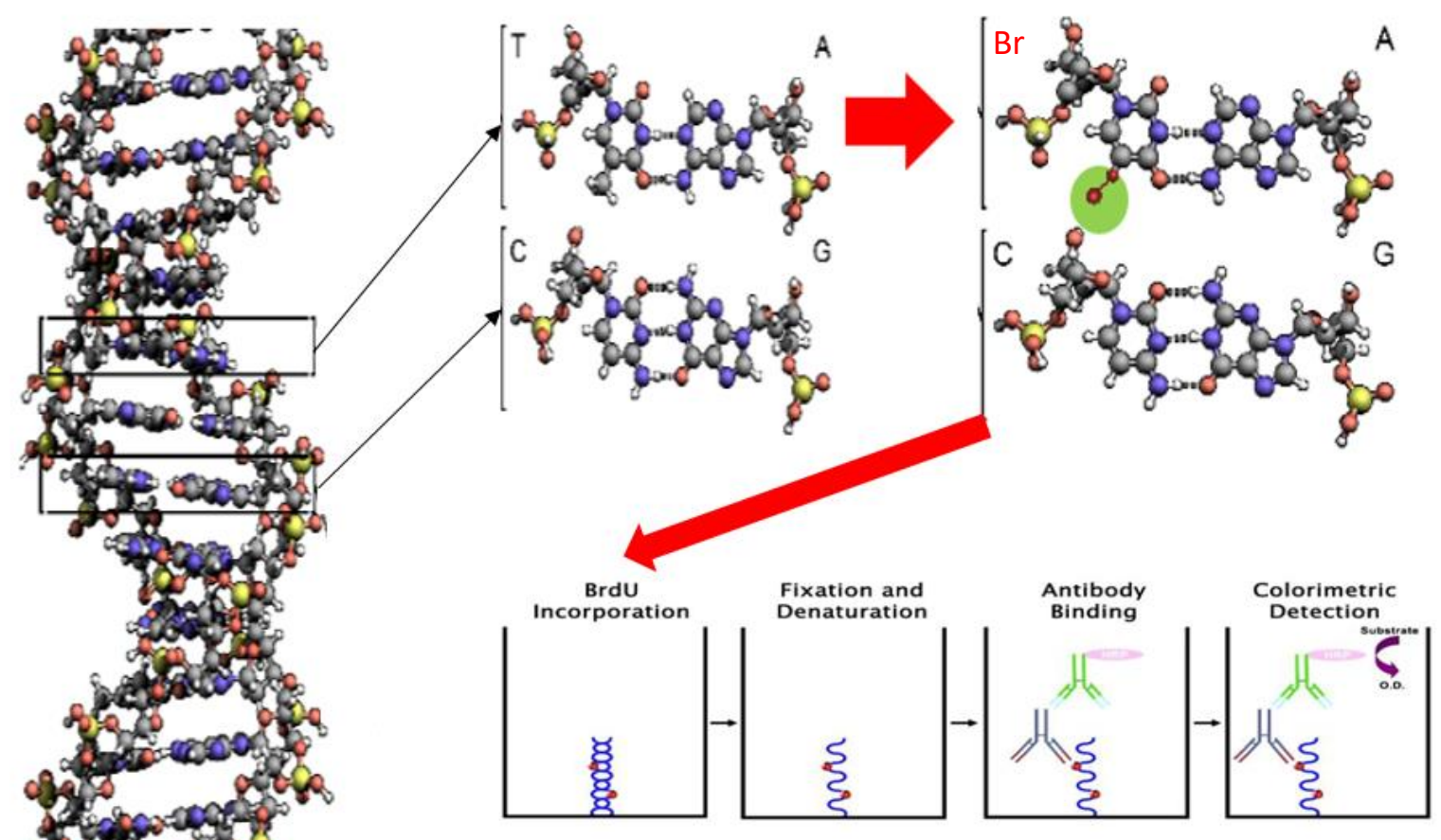




\section{Method}

Animals

A separate cohort of animals was used for this experiment which consisted of 13 litters of pups produced from pairing seven different genotype combinations to produce all possible genotypes in the offspring and all different maternal genotypes. However, for the purpose of this study, only male pups produced from SERT ${ }^{+/-}$dams will be examined to limit maternal influences, thus only animals fitting this criterion will be discussed from this point (litters: 5, pups: 26). All animals were housed in the Victoria University of Wellington vivarium in accordance with the Animal Welfare Act 1999, with all procedures being approved by the VUW Animal Ethics Committee (AEC number 22375). All animals were housed in polycarbonate cages (one dam and her offspring per cage) in a temperature and humidity (55-60\%) controlled environment, $21^{\circ} \mathrm{C} \pm$ 2 and 55-60\% respectively. A reverse light cycle was used (lights on at 1900-0700 hours), consistent with all other experiments.

\section{Tissue Preparation}

At postnatal day 7 (PND 7), pups were removed in pairs and taken to a separate room to avoid stress on the remaining animals. After weighing, pups were injected with $25 \mathrm{mg} / \mathrm{kg}$ of BrdU intraperitoneally, marked for identification, and returned to the dam within 5 minutes. Following a 2-hour integration phase, the animals were rapidly decapitated. Tail clippings were taken to establish the animal's genotype (Transnetyx, Inc.), and brains were submerged in $4 \%$ paraformaldehyde solution for 24 hours for protein fixing, following this, brains were immersed in $30 \%$ sucrose solution for cryoprotection. Once the brains showed no buoyancy, indicating the complete transfer of water for sucrose solution had occurred, the brains were snap frozen at -40 ${ }^{\circ} \mathrm{C}$ in isopentane, at which point they were stored at $-70--80{ }^{\circ} \mathrm{C}$ until sectioning. All brains were coded at this point to keep the experimenter blind for the remained of the experiment. Sectioning was completed using a freezing microtome, whereby $30 \mu \mathrm{m}$ sections were obtained, with every 
sixth section being used in the analysis to ensure a $180 \mu \mathrm{m}$ difference between sections to avoid counting the same cell multiple times (Coordinates; P7, C11-13).

\section{BrdU Labelling Procedure}

The ABC peroxidase method for labelling BrdU positive cells in free floating sections, a procedure previously established within our lab group, was used in this experiment. All steps were conducted under mild agitation at room temperature. Sections were removed from the Phosphatebuffered saline (PBS) azide solution in which they were stored, and washed three times, for five minutes in PBS (10 mM, pH 7.4) (replaced between each wash). An antigen retrieval process was used to break the protein crosslinks formed during most standard fixation methods, allowing for greater staining intensity; the sections were incubated for 90 minutes in $5 \mathrm{ml}$ of $2 \mathrm{~N}$ hydrochloric acid (HCL). Following incubation, sections were washed three times in PBS as before. Sections were incubated in 1\% Bovine Serum Albumin (BSA, Sigma-Aldrich) for 60 minutes to block nonBrdU binding sites, preventing non-specific antibody binding for occurring. Following incubation, as previously, sections were washed using PBST, created by adding Triton (X-100, ThermoFisher) to PBS solution (1:1000). Next, sections were incubated in 3\% hydrogen peroxide (H2O2) for 30 minutes to induce membrane permeability, then washed in PBST solution. To increase the number of binding locations per BrdU positive cells, sections were incubated overnight in the primary antiBrdU mouse monoclonal antibody, conjugated to biotin (ab2284, Abcam). Following this incubation period, sections were washed in PBST, then incubated in Avidine-biotine peroxidase complex (ABC, Vectastain) for 60 minutes. Finally, following washing in PBST, sections were incubated in 3,3'-diaminobenzidine (DAB; $4 \mathrm{mg} / \mathrm{ml})$, Nickel chloride (NiCl2; $8 \mathrm{mg} / \mathrm{ml})$ (2:1) solution with $0.3 \% \mathrm{H} 2 \mathrm{O} 2$ to induce oxidisation resulting in a rust coloured stain binding to the BrdU sites.

Following staining, sections were kept at $4{ }^{\circ} \mathrm{C}$ until mounting within 48 hours. Stained sections were extracted from the baskets and immersed in PBST and gently mounted onto the 
gelatinised slides and allowed to dry. Following mounting, counterstaining was completed to provide a strong contrast between BrdU positive cells and the surrounding tissue (see figure 4). This procedure involves immersion of the mounted sections in 1\% neutral red assay (Biovision) for 2 minutes, followed by a brief immersion in a progressively increasing strength of ethanol solution $(70 \%, 95 \% \mathrm{x} 2,100 \% \mathrm{x} 2)$. Following this, sections were immersed in two baths of Histo-Clear (National Diagnostics), then a coverslip was applied with DPX (Sigma-Aldrich) and allowed to dry for at least 48 hours.

Figure 4. Representative section with the dentate gyrus region mapped

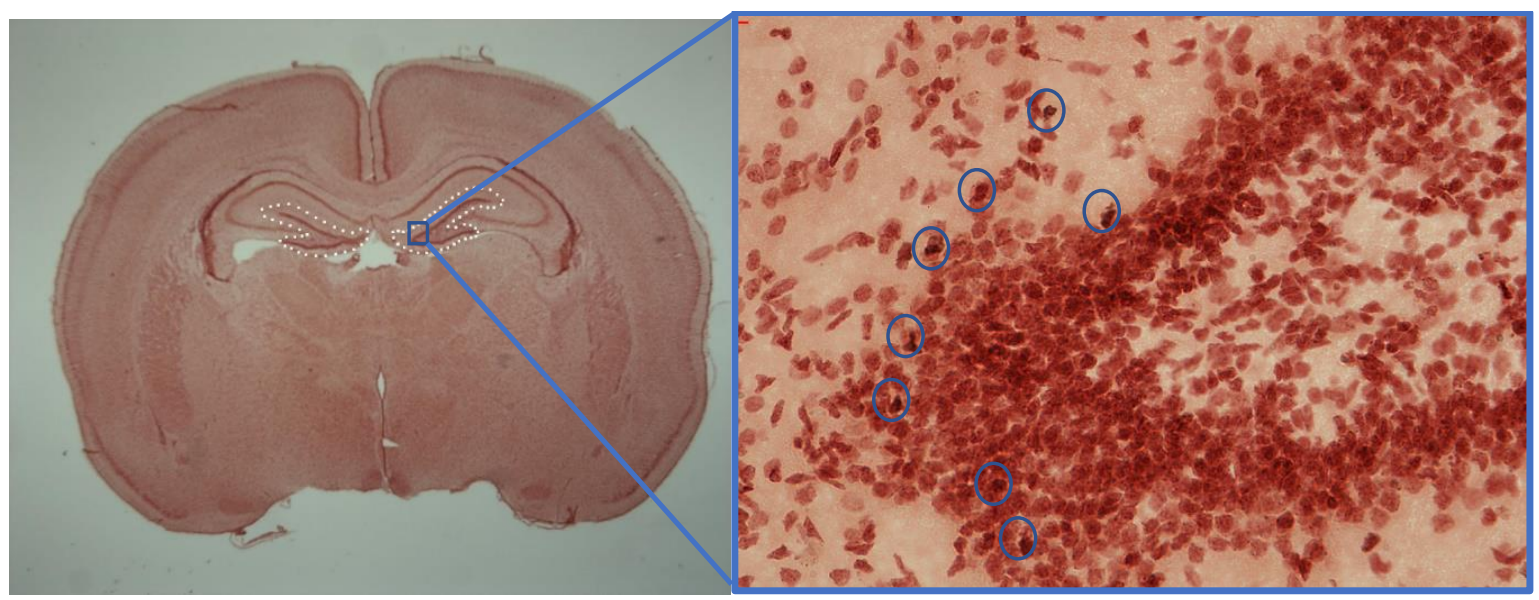

\section{Results}

Means and standard deviations of the total area $\left(\mu^{2}\right)$ of dentate gyri used in the analysis were computed for SERT ${ }^{+/+}(M=45.52, S D=11.83), \mathrm{SERT}^{+/-}(M=46.67, S D=5.62)$, and SERT $^{-/-}(M=49.46, S D=6.74)$, to explore the equivalence of the samples between groups. Equivalence of the size of the dentate gyri between genotypes was determined through a one-way ANOVA which revealed no significant differences $[F(2,11)=.227, p=.801]$, ensuring no bias underlying the areas examined during data collection (Table 1). Following the confirmation of equivalence, means and standard deviations for the density of BrdU positive cells were produced for SERT ${ }^{+/+}(M=0.32, S D=0.30)$, SERT $^{+/-}(M=0.75, S D=0.09)$, and SERT ${ }^{-/}(M=1.45, S D$ $=0.70)$ (figure 5). A one-way ANOVA showed a significant genotype effect $[F(2,11)=6.65, p=$ 
.017]. A post hoc Tukey test was conducted to identify the origin of the differences; SERT ${ }^{+/+}$and $\mathrm{SERT}^{-/}$differed significantly $(p=.014)$, while no other differences withstood correction for multiple tests. These results indicate that $\mathrm{SERT}^{-/-}$animals show more neurogenesis than $\mathrm{SERT}^{+/+}$ animals during early development.

Table 1. Density (BrdU positive cells per $\mu^{2}$ ) and hippocampal area

Density of BrdU Positive Cells

\begin{tabular}{ccccccc}
\hline \hline & \multicolumn{2}{c}{ SERT $^{+/+}$} & \multicolumn{2}{c}{ SERT $^{+/-}$} & \multicolumn{2}{c}{ SERT-/- } \\
\hline & Area $\left(\mu^{2}\right)$ & Density & Area $\left(\mu^{2}\right)$ & Density & Area $\left(\mu^{2}\right)$ & Density \\
\hline Mean & 45.52 & 0.32 & 46.67 & 0.75 & 49.46 & 1.45 \\
Standard Deviation & 11.83 & 0.3 & 5.62 & 0.09 & 6.74 & 0.7 \\
\hline
\end{tabular}

Figure 5.

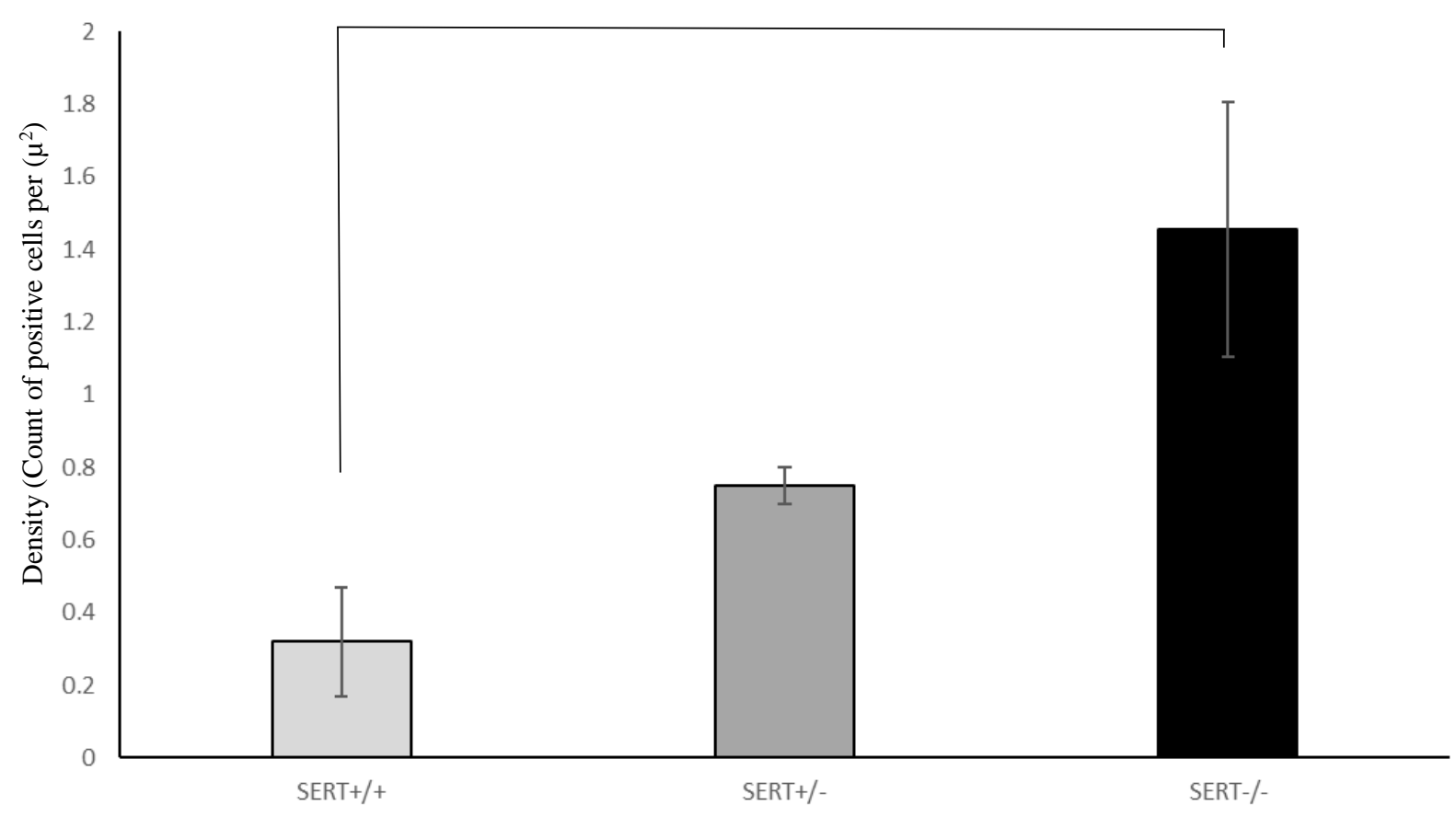

Figure 5. Means and \pm SEM for the density of BrdU positive cells indicating the number of new cells developed following BrdU administration (2 hours).

* Indicates significant differences $(p<.05)$ 


\section{Discussion}

The present experiment sought to examine the impact of a genetic reduction of serotonin transporter expression on neurogenesis during early development. Prior studies have indicated a modulatory role of serotonin in neurogenesis, particularly in adulthood, with common antidepressants increasing neurogenesis in adulthood through the increased extracellular serotonin, a process thought to underlie the reduction in depressive symptom severity. However, other studies have clearly shown that increase in serotonin in early development are actually a risk factor for the development of psychiatric disorders, including depression (Homberg et al., 2010). To examine this serotonin paradox, we set out to investigate the impact of increased serotonin during early development, the rate of neurogenesis was examined in $\mathrm{SERT}^{+/+}, \mathrm{SERT}^{+/}$and SERT /. rats using BrdU. It was hypothesised that the proportion of BrdU positive cells would be increased in $\mathrm{SERT}^{-/}$in comparison to the $\mathrm{SERT}^{+/+}$group due to the promotion of neurogenesis through serotonin. Furthermore, the partial reduction of serotonin transporter expression as seen in the SERT ${ }^{+/-}$group was hypothesised to produce a smaller increase in BrdU than the $\mathrm{SERT}^{-/}$, when compared to the $\mathrm{SERT}^{+/+}$group, thus producing a gene-dose response. These hypothesise were supported as there was a significant increase in BrdU positive cells in the hippocampus of $\mathrm{SERT}^{-/-}$rats, compared to the $\mathrm{SERT}^{+/+}$animals, with the $\mathrm{SERT}^{+/-}$group being intermediate to both.

Given the significant increase in neurogenesis observed in the SERT ${ }^{-/}$animals used in this experiment, it is clear that developmental changes are occurring in response to the increased extracellular serotonin. Given that such new born cells are likely to make new synaptic contacts, this early increase in serotonin signalling is likely to change the connectivity within the hippocampus. Whether this also occurs in other brain regions has yet to be investigated. However, since neurogenesis at this age (and especially earlier) also occurs in multiple other brain regions, and $\mathrm{SERT}^{-/-}$have increased serotonin levels throughout the brain, this seems highly likely. In 
addition to the results of the current study, high levels of serotonin during development have also been shown to induce changes in the barrel cortex and the frontal cortex.

The barrel cortex, a rodent specific portion of the somatosensory cortex critically involved receiving sensory information has been intensively studied due to the simplistic structure and topographical layout (Petersen, 2007). The role of serotonin during development heavily impacts the formation of the barrel cortex, whereby depletion of serotonin results in significant developmental stunting (Persico et al., 2000), while excess levels as shown in the monoamine oxidase-A deficient mice have been observed to not show the characteristic barrel shaped clusters (Cases et al., 1996). These data demonstrate that serotonergic homeostasis is required for normal cortical development and cytoarchitecture, whereby increased serotonin produces structurally unorganised barrel formation, while depletion stunts development typically requiring serotonergic mediation. While the frontal cortex is a more complex structure, the role of serotonin during early development is equally important. Numerous deficits have been observed in the prefrontal cortex in response to increased extracellular serotonin during critical stages of development. For example, (Witteveen et al., 2013) demonstrated significant alterations to the development of the rapheprefrontal network is SERT knockout rodents, an effect suggested to occur in response to guidance cues. As discussed earlier, excess serotonin resulted in the reversal of the netrin-1 cues involved in axon guidance (Bonnin et al., 2007), similarly, Witteveen et al. (2013), demonstrated the reversed guidance effect of the median raphe serotonin neurites in $\mathrm{SERT}^{-/}$rats. These data indicate the significant role of serotonin during critical stages of development whereby challenging the homeostasis results in altered guidance cues which, in turn result is abnormal cytoarchitecture. Similarly, these serotonin mediated deficits have been observed in humans with psychiatry illness, particularly autism spectrum disorder, thus demonstrating the role of serotonin innervation of the frontal cortex (Schubert, Martens, \& Kolk, 2015). 
It is, at present difficult to identify the exact molecular mechanism underlying this increase in neurogenesis during development, or indeed the other developmental changes. Studies have shown in $\mathrm{SERT}^{-/-}$rats, 5-HT ${ }^{1 \mathrm{~A}}$ receptors are downregulated in adulthood (J. D. Olivier et al., 2008). Additionally, we have unpublished data of downregulated 5-HT2C receptors and it seems highly likely that other 5-HT receptors are also downregulated. However, exactly when this downregulation occurs is unknown. Thus, it is entirely feasible that at postnatal day $7,5-\mathrm{HT}^{1 \mathrm{~A}}$ and 5- $\mathrm{HT}^{1 \mathrm{~B}}$ receptors are not yet downregulated and thus (as mentioned above) enhanced stimulation of these receptors would induce enhanced neurogenesis. In adulthood, when downregulation has occurred, an under-stimulation of these receptors may then lead to the reduced neurogenesis seen in major depression. However, other factors may also play an important role. For instance, it is well known that increased cortisol release can influence neurogenesis (Gould \& Tanapat, 1999), and some studies have shown that humans with the s-allele of the 5-HTTLPR have increased hypothalamus-pituitary-adrenal (HPA) axis activity, leading to an altered circulation cortisol levels.

For instance, human infants expressing the s allele of the 5-HT'TLPR polymorphism show increased cortisol response to stress, and a marginally significant increase in baseline cortisol irrespective of environmental factors. Given the negative influence of cortisol on neurogenesis, this may in part counteract the effects of increased serotonin levels. It is tempting to speculate that this may explain the lack of effect in the SERT ${ }^{+/-}$rats, but clearly more research is needed.

In summary, the present study sought to examine the impact of increased serotonin levels on neurogenesis during early neurodevelopment in order to gain insight into the mechanisms underlying the serotonin paradox. Through the genetic reduction in the expression of serotonin transporters, we found a gene-dose related increase in neurogenesis in the dentate gyrus of the hippocampus. Although further studies are needed to investigate to what extent this finding can be extrapolated to other brain regions, the data do show that the brains of SERT ${ }^{-/-}$rats are fundamentally different from SERT ${ }^{+/+}$rats. Together with changes found in the serotonin 
innervation of the frontal cortex (Schubert et al., 2015), and even changes in the dopaminergic system, this begs the question how different these brain are. While it would be impossible to answer this in a single master's thesis, in the next chapter we introduce a relatively novel technique to investigate regional changes in multiple biochemical compounds in a single sample. 


\section{CHAPTER SIX}

Experiment Five

Piloting the Use of Matrix Assisted Laser Desorption/Ionisation on Brain and

Heart Tissue 
Immunohistochemistry has been a great contributor to many fields of research, from oncology to psychiatry (Wood, 2014), however, one core component of these techniques is their limited scope. While it is possible with some techniques to process the same tissue multiple times, each procedure is highly specified to detection a specific protein such as brain derived neurotropic factor (BDNF), or a specific cell phase such as KI-67 or BrdU. Given the specificity of these techniques, the research conducted tends to be labour intensive and primarily driven by on a priori hypotheses and it seems unlikely that such techniques lead to the identification of completely novel insights.

Technological advances have provided a range of new methods, which through slight modifications can be appropriated into new fields which were not originally intended to use those methods. For example, mass spectrometry (MS) was initially theorized and developed (while crudely) in the late $19^{\text {th }}$ century. Since then, MS has undergone many changes, with novel techniques developed for the specific application in a particular field of research. MS has the advantage of being able to detect multiple (in theory hundreds of) compounds (including even unknown) in the same sample. While this is useful for a relatively homogeneous sample (such as serum or urine), it is inherently less informative when studying the brain, where concentrations of compounds vary strongly between different brain (sub)regions. Here we present a relatively novel method for examining all possible compounds in the brain from neurotransmitters to proteins with a high spatial resolution. While in theory this technique would allow to investigate all chemical changes in for instance the SERT-/- rats (even across multiple time points during development), the present study is merely designed to provide a proof-of-principle.

\section{MALDI theory}

Matrix assisted laser desorption ionisation time-of-flight MS (MALDI-TOF MS), integrates aspects of MS, however through the use of a laser absorption matrix, the molecules remain more stable and less fragmentation occurs (Medzihradszky et al., 2000). Firstly, it is 
important to understand the fundamental theory of time-of-flight MS (TOF MS), thus this will be briefly described; TOF MS involves directing electrons through an analyte, transferring the kinetic energy into the sample, this in turn deconstructs the sample into its ions which travel towards a detection point. Based on the mass to charge ratio, these ions will contain the same charge, but a different mass, with larger particles travelling at a slower rate, and thus being detected later. This then leads to a spectrum containing peaks at different time points. The height of the peak correlates with the concentration of the compound, with the position of the peak within the time spectrum being indicative of the identity of the compound. The primary limitation of conventional MS procedures is the fragmentation which occurs due to the intensity of the laser required to ablate the sample resulting is significant noise surrounding the peak of interest.

To reduce the fragmentation, MALDI incorporates a matrix which contains a chromophore which absorbs the laser, dispersing the intensity evenly over the sample. This absorption allows for the use of a lower laser intensity to induce ablation (i.e. soft ionisation); often a $337 \mathrm{~nm}$ wavelength nitrogen laser (Medzihradszky et al., 2000). While this method has been shown to reduce fragmentation, primarily within large compounds such as proteins, significant fragmentation has been noted in low mass compounds such as primary amines (Shariatgorji et al., 2015). Furthermore, conventional matrices are often of a lower mass composition producing a significant masking effect of the lower mass range. Due to this masking of lower mass ranges, the MALDI technique has been of little value within neuroscience, as quantification of (mostly small molecule) neurotransmitters such as dopamine and serotonin has not been possible.

Recent developments have incorporated a derivatisation agent into the conventional MALDI technique with the results suggesting a reduced fragmentation and increased intensity of smaller compounds, resulting in the ability to quantify neurotransmitters such as dopamine (Shariatgorji et al., 2014). One derivatisation agent, 2-4-6-Triphenylpyrylium tetrafluoroborate (TPP), derivatives compounds containing an amino group (NH2), a defining component of 
amines, converting the amine into a pyrylium salt structure (Shariatgorji et al., 2015). This pyrylium salt conversion is advantageous firstly due to providing additional stability to amines, resulting in less fragmentation, while secondly significantly increasing the mass of the amine, resulting in detection at a mass above the masking range of most matrices.

Through this method, we propose that it is possible to detect a variety of amines, as well as other smaller compounds containing an amino group. Moreover, by moving the laser across a brain slice, it would be possible to obtain an MS spectrum for every $50 \mu \mathrm{m}^{2}$ of tissue, thus allowing for a high spatial resolution. Together this would make it possible to examine changes in concentrations of a wide variety of neurotransmitters throughout the tissue sample (and in theory throughout the brain. The present experiment sought to pilot the use of MALDI incorporating the use of TPP as a derivatisation agent on brain tissue to accurately detect a broad range of compounds. Furthermore, we tested the versatility of this technique by simultaneously examining cardiac tissue.

\section{Method}

Tissue preparation

After decapitation, brain tissue from an adult male Wistar rat was rapidly extracted and snap frozen using liquid nitrogen (see appendix 10). Samples were transferred to a cryostat $\left(-20_{-}^{\circ}\right.$ C) and allowed to acclimatise for 20 minutes to improve sectioning quality. Following acclimatization, sections were halved down the longitudinal fissure, at which point the right hemisphere was mounted on OCT. Sections were trimmed to create an even cutting surface, following which, sagittal sections were obtained at $10 \mu \mathrm{m}$ thickness. Sectioned were placed on the MALDI target slides (maintained at $-20^{\circ} \mathrm{C}$ ), after which a heat source was briefly applied below the sample location resulting in rapid tissue adherence to the sample slide, thus avoiding residual ice crystal formation and loss of water soluble compounds associated with gradual adherence. 
Following sectioning, slides were dried at room temperature for 30-60 minutes using a desiccator to prevent condensation forming and delocalisation of compounds. The sample slide was then imaged in high definition to align the final spectra output and the sample. To validate the accurate alignment of the spectra, reference points were added using a $1 \mathrm{mg} / \mathrm{ml}$ spot of dopamine (Dopamine hydrochloride (Sigma-Aldrich) dissolved in distilled water), and $1 \mathrm{mg} / \mathrm{ml}$ of serotonin (Serotonin hydrochloride (Sigma-Aldrich) dissolved in distilled water), spotted near the tissue.

In the next step, the derivatisation agent, 2-4-6-Triphenylpyrylium Tetrafluroborate (TPP, $1.8 \mathrm{mg} / \mathrm{ml}$, diluted in a 70\% methanol and $0.5 \%$ Trithylamine solution). TPP was selected due to its ability to react with amines creating pyrylium salt structures. The derivatisation agent was evenly applied over the sample (Flow rate; $0.02 / \mathrm{ml}$ per minute, 250 PSI, $70-75_{-}^{\circ}$ C) using an automatic sprayer. The sample was incubated in a beaker elevated over $4 \mathrm{ml}$ of 50\% methanol solution for 60 minutes. During this incubation phase, the derivatisation agent reacts with the amines through creating a basic environment, opening the chemical structure, and allowing for the formation of the pyrylium salt structure. Due to the acidic nature of the matrix, there was no need to re-acidify the sample as seen in prior studies (Kamath, Diedrich, \& Hindsgaul, 1996), as this was likely to increase delocalisation. Finally, $\alpha$-cyano-4hydroxycinnamic acid (CHCA, Sigma-Aldrich, $8 \mathrm{mg} / \mathrm{ml}$ was created through combining in a 50\% solution of acetonitrile and $0.2 \%$ Trifloroacetic acid) was used as the matrix due its broad use in soft ionisation techniques. The matrix was applied using the automatic sprayer $\left(75{ }_{-}^{\circ} \mathrm{C}\right)$ with the TPP acting as a proton donor allowing for the absorption of the laser resulting in the ionization and abolition of the sample tissue. Finally, the sample was incubated with $4 \mathrm{ml}$ of $50 \%$ methanol solution and $0.2 \mathrm{ml}$ of acetic acid.

Following CHCA application, the sample slide was loaded into the Mass-spectrometer (AB sciex tof/tof 5800), which uses a variable laser set at $337 \mathrm{~nm}$ in a vacuum chamber to process the spectra using the time of flight principle. The laser ablates and ionises the sample with a resolution of $50 \mu \mathrm{m}^{2}$ per pixel. Following the completion of the MALDI procedure, the output spectra were analyses using Bitmap software. 


\section{Results}

Due to the translucence of the tissue, exact coordinates were unable to be established, however, the sagittal brain section appeared to be between lateral $-0.10 \mathrm{~mm}$ and $0.50 \mathrm{~mm}$ coordinates (Paxinos \& Watson, 1998) (see figure 1). Given the nature of the experiment (i.e a proof of principle), no quantification was attempted, but rather a qualitative approach was used to determine the validity of the method for studying the chemistry of cardiac and brain tissue.

Figure 1. Tissue images taken following desiccation

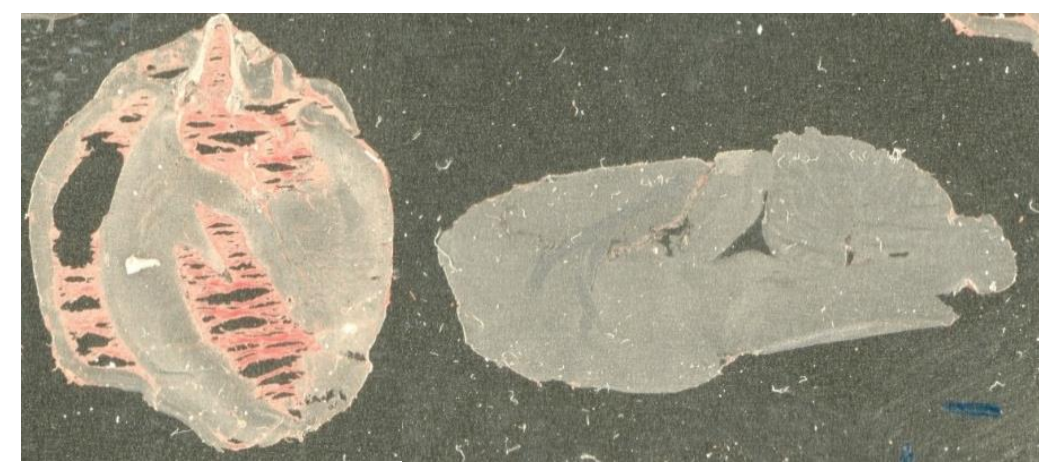

Brain tissue was initially examined for the presence of dopamine, serotonin and GABA. These results will be discussed below.

Figure 2. TPP Average spectra intensity at 308.94-309.13 g/mol

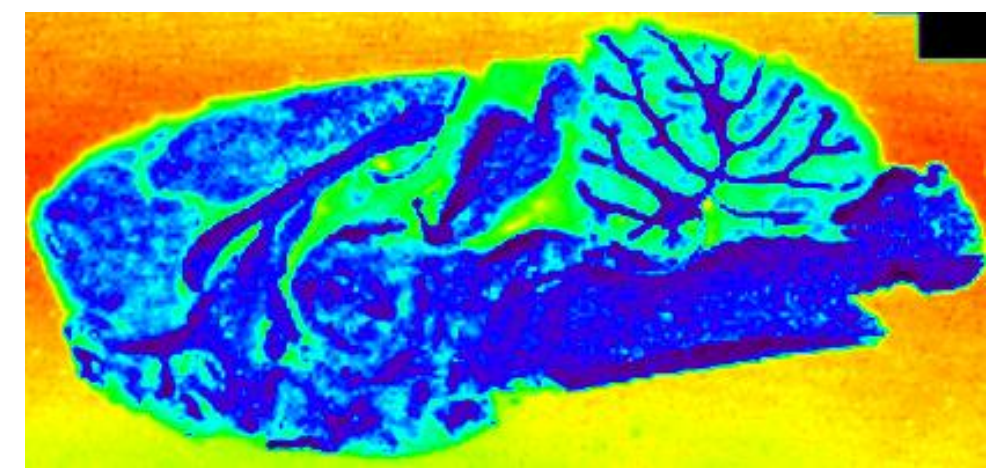

Based on prior experiments from our laboratory, the detected mass of TPP is centred around 309 $\mathrm{g} / \mathrm{mol}$ due to the loss of the tetrafluoroborate, thus this mass acts as a reference point and allows for the calculation of a pre-derivatization mass of detected peaks of interest. As unbound TPP 
could be detected throughout the tissue, but was of lower intensity than the surrounding plate surface (figure 2), it can be concluded that adequate derivatisation had occurred.

Figure 3. Dopamine Average spectra intensity at 308.94-309.13 g/mol

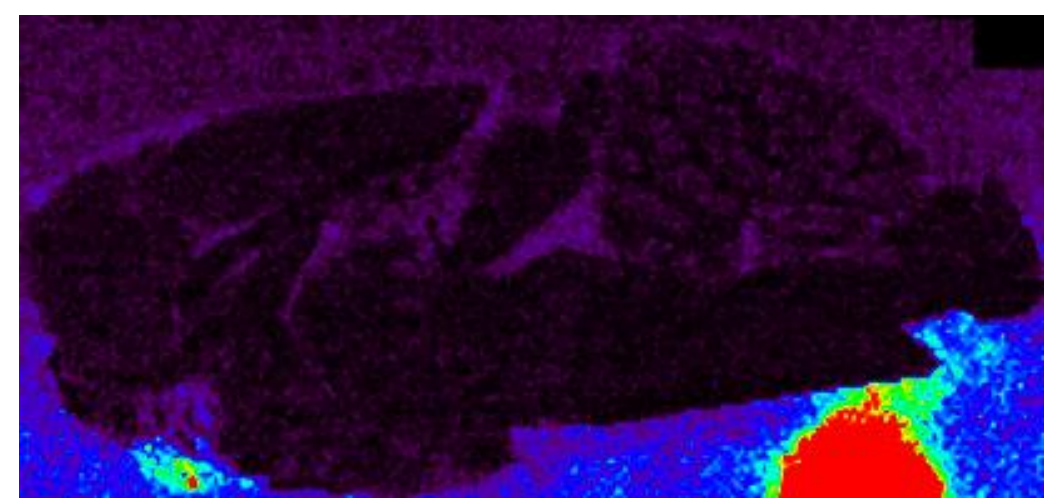

As a known quantity of dopamine was spotted near the tissue, the mass peak of dopamine could be identified through targeting this point. This peak was centred around $444 \mathrm{~g} / \mathrm{mol}$, consistent with prior observations within our laboratory. To establish the accuracy of this detection, the observed mass of TPP (following the deduction of $\mathrm{H} 2 \mathrm{O}$ formed during the formation of the pyrylium salt structure) is deducted from the observed mass of dopamine (444 $291.11 \mathrm{~g} / \mathrm{mol}$ ) to produce an observed pre-derivatisation mass of $152.89 \mathrm{~g} / \mathrm{mol}$ (virtually identical with the molecular weight of dopamine (Figure 4), (153.2 g/mol) Examining the distribution of dopamine shows an unexpected absence of dopamine throughout the tissue, aside from a cluster of high intensity localised around the optic chiasm region (figure 3). 
Figure 4. Dopamine reaction with TPP to form pyrylium salt structure

a)

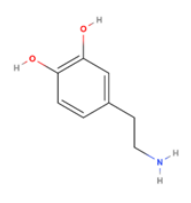

Dopamine

$\left(\mathrm{C}_{8} \mathrm{H}_{11} \mathrm{NO}_{2}\right)$

$153.178 \mathrm{~g} / \mathrm{mol}$ b)

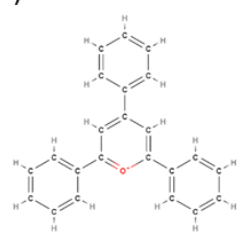

$\left(\mathrm{C}_{23} \mathrm{H}_{17} \mathrm{BF}_{4} \mathrm{O}\right)$

$396.192 \mathrm{~g} / \mathrm{mol}$ c)

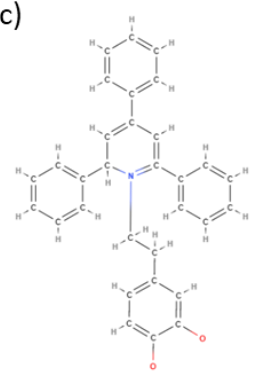

Dopamine - pyrylium salt $\left(\mathrm{C}_{8} \mathrm{H}_{9} \mathrm{NO}_{2}\right)+\left(\mathrm{C}_{23} \mathrm{H}_{17} \mathrm{O}\right)$ $444 \mathrm{~g} / \mathrm{mol}$ d)

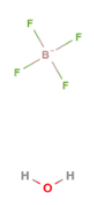

By-products $\mathrm{H}_{2} \mathrm{O}$ and $\mathrm{BF}_{4}$ $86.803+18.153 \mathrm{~g} / \mathrm{mol}$

Figure 4.. $\quad$ The reaction between the compound of interest and TPP. a) Compound of interest, b) TPP prior to reaction, c) detected compound, d) by-products of the reaction.

Figure 5. Serotonin; Average spectra intensity at $467.04-467.224 \mathrm{~g} / \mathrm{mol}$

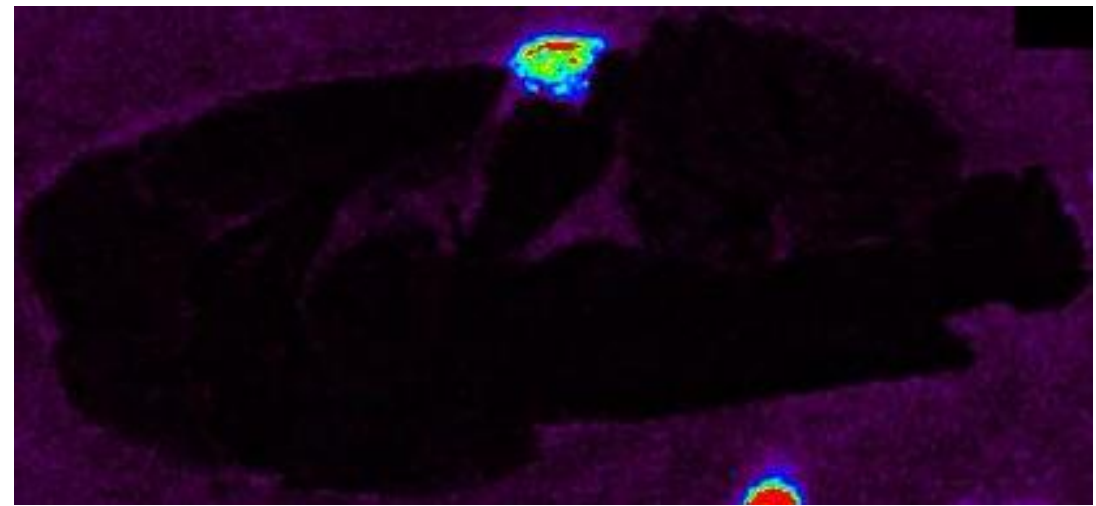

Using the same technique, serotonin was spotted a peak at $467 \mathrm{~g} / \mathrm{mol}$ was identified, corresponding to a molecular weight of $175.89 \mathrm{~g} / \mathrm{mol}$ for the underived form of serotonin (molecular weight $176.2 \mathrm{~g} / \mathrm{mol}$ ). Serotonin shows a high intensity located within the region containing the pineal gland, while no detectable level of serotonin was observed in any other tissue region (figure 5). 
Figure 6. GABA; Average spectra intensity at $393.990-394.160 \mathrm{~g} / \mathrm{mol}$

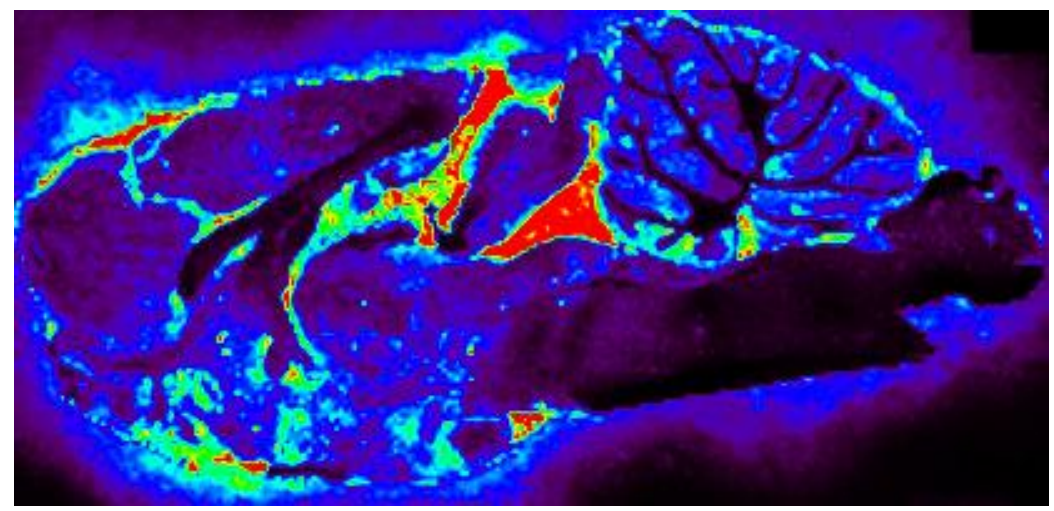

Due to the accuracy of the calculations used in the detection of dopamine and serotonin, this formula was reversed to determine whether it could be used as a predictive calculation. GABA was selected as a target amine due to containing the required NH2 group for derivatisation, and the relatively broad distribution throughout the brain. GABA has a theoretical mass of 103.12 $\mathrm{g} / \mathrm{mol}$, thus examination of the predicted mass of the pyrylium salt structure formed through derivatisation with TPP $(394.23 \mathrm{~g} / \mathrm{mol})$ was conducted. A spectra peak was observed at $394 \mathrm{~g} / \mathrm{mol}$, which was determined to be GABA due to being within the tolerance range of this procedure (difference of $0.23 \mathrm{~g} / \mathrm{mol}$ ). A relatively broad localisation of GABA was identified, with high intensity detected around hypothalamic, basal forebrain, midbrain regions (see fig ๑).

\section{Cardiac tissue}

Thus far we have established the ability to detect amines within brain tissue to a high degree of accuracy using MALDI with TTP as a derivatisation agent and CHCA as a Matrix. To determine the versatility of this method, cardiac tissue was subjected to the same treatment.

Figure 7. TPP; Average spectra intensity at $309.045-309.271 \mathrm{~g} / \mathrm{mol}$ 
a)

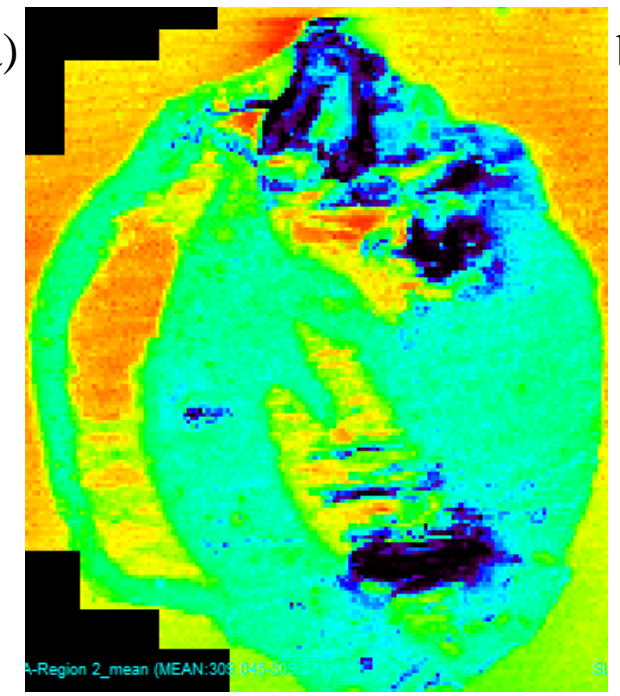

b)

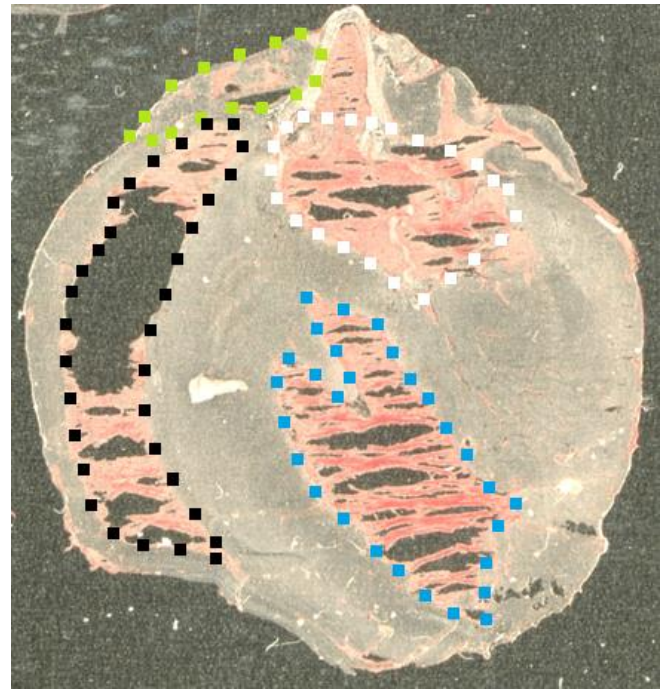

Figure $7 . \quad$ a) Intensity distribution of TPP in cardiac tissue. b) Cardiac tissue with core regions highlighted; white: right atrium, green: left atrium, black: right ventricle, blue: left ventricle

Figure $7 \mathrm{a}$ depicts the detection of TPP within the cardiac tissue at $309 \mathrm{~g} / \mathrm{mol}$. As with brain tissue, a sufficient degree of unbound TPP indicates ample derivatisation throughout the tissue. It should be noted that within some of the residual blood (figure 7b), TPP was undetectable which suggests that all TPP was bound, thus supressed peaks may be present within these regions. No regions showed intensities equivalent to the surrounding sample plate, thus, similar to the brain, TPP seems to be an effective derivative tool for cardiac tissue.

\section{Exploratory compound identification}

Due to the ability to predict a known amine within the brain using the reversed formula (as with GABA), this method was applied to determine the viability of this approach as an exploratory compound identifier. The spectra was examined, with three mass peaks identified due to their clear peak intensities and patterns of localisation within the tissue sample. The identification method and results will be described below: 
Figure 8. Dopamine 3-O-sulfate; Average spectra intensity at $524.538-524.587 \mathrm{~g} / \mathrm{mol}$

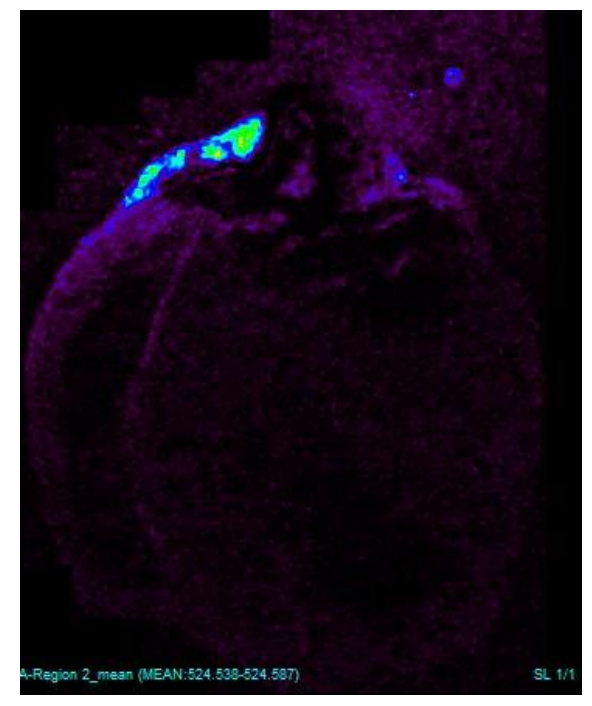

A highly localised compound was detected at $524.5 \mathrm{~g} / \mathrm{mol}$, thus following the previously established formula, the pre-derivatisation compound was predicted to have a mass of 233.39 $\mathrm{g} / \mathrm{mol}$ (figure 8). Following the identification criteria, MassBank produced 57 results, mzCloud identified 23 potential compounds, HMDB identified 23 compounds within the tolerance range, while Metlin identified over 2000 potential compounds. From these results, all compounds not containing a NH2 component were excluded, and, of the remaining compounds, all purely exogenous compounds were excluded. The significantly reduced number of compounds were subjected to a literature review to determine the most likely option. Based on this, it was concluded that the detected compound was most likely to be Dopamine 3-O-sulfate (theoretical mass: $233.242 \mathrm{~g} / \mathrm{mol}$ ). Only Metlin and HMDB identified Dopamine 3-O-sulfate in the original search. From the literature, dopamine 3-O-sulfate, the sulfonated form of dopamine, most prominent in the peripheral nervous system, appears to be highly localised around the sympathetic postganglionic fibre in the right ventricle (Lokhandwala \& Barrett, 1983). 
Figure 9. Deoxyadenosine triphosphate; Average spectra intensity at $782.566-782.806$

$\mathrm{g} / \mathrm{mol}$

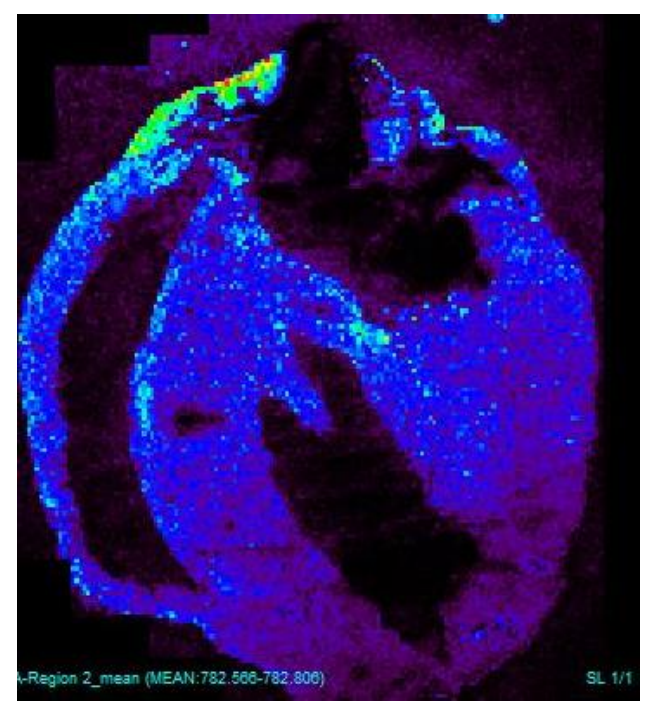

A second compound showing a distinctive pattern of high and low intensity localisation was detected at $782.5 \mathrm{~g} / \mathrm{mol}$ (see fig 9). If the compound contained an NH2 component allowing TPP binding, the pre-derivatisation compound was predicted to have a mass of $491.39 \mathrm{~g} / \mathrm{mol}$. The mass was centred into Massbank (1), mzCloud (2), HMDB (4), and Metlin (1475). Those endogenous compounds containing a NH2 group were subjected to a literature review to determine the most likely to explain the localisation pattern. It was determined that the target compound was most likely to be Deoxyadenosine triphosphate (dATP) (theoretical mass: 497.782 $\mathrm{g} / \mathrm{mol}$ ) (See table 1). All data bases aside from Massbank identified dATP within the original search criteria. Localisation occurs within the right atrium, then diffuses gradually throughout the tissue (Cheng, Hogarth, O'Sullivan, Regnier, \& Pyle, 2015). 
Figure 10. Histamine: Average spectra intensity at $401.966-402.042 \mathrm{~g} / \mathrm{mol}$

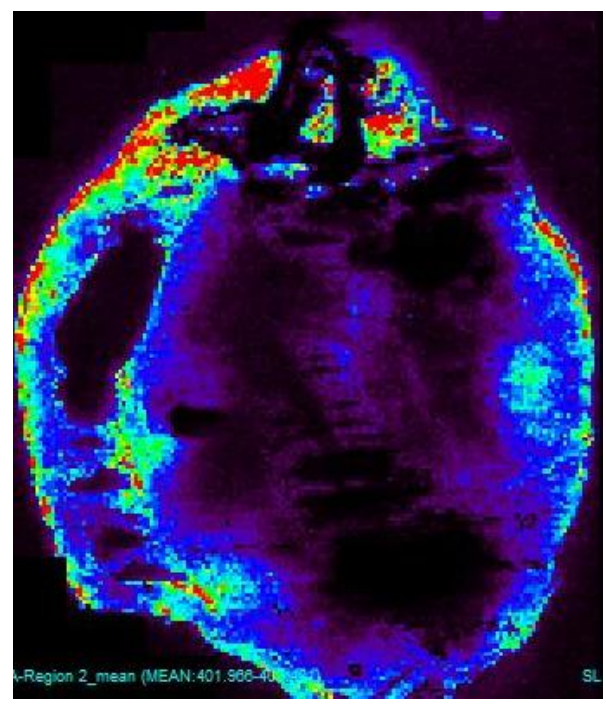

Finally, a compound showing high intensity diffuse labelling was detected at $402 \mathrm{~g} / \mathrm{mol}$. Massbank (58), mzCloud (5), HMDB (18), and Metlin (479) all produced compounds around $110.89 \mathrm{~g} / \mathrm{mol}$, of which based on the same method as previously described, histamine was selected as the most likely compounds (theoretical mass: $111.145 \mathrm{~g} / \mathrm{mol}$ ) to explain the pattern of distribution (figure 10). This compound is detected primarily in the external tissue (Matsuda et al., 2004). 
Table 1.

Summary of Identification Criteria and Results

\begin{tabular}{|c|c|c|c|c|c|c|c|c|c|c|}
\hline Tissue & Detected Mass & Undervivitized Mass & Compound Search Range & Compounds within Range & Identified in initial search & Selected Compounds & Colloquial Name & Formula & Theoretical Mass & Difference \\
\hline $\begin{array}{l}\text { Brain } \\
\text { Heart } \\
\end{array}$ & $309 *$ & N/A & N/A & N/A & N/A & 2-4-6-Triphenylpyrylium & TPP & $\mathrm{C} 23 \mathrm{H} 17 \mathrm{O}$ & 309.392 & 0.392 \\
\hline Brain & $444^{*}$ & 152.89 & N/A & N/A & N/A & 3,4-dihydroxyphenethylamine & e Dopamine (DA) & $\mathrm{C} 8 \mathrm{H} 11 \mathrm{NO} 2$ & 153.178 & 0.288 \\
\hline Brain & $467 *$ & 175.89 & N/A & N/A & N/A & 5-Hydroxytryptamine & $\begin{array}{c}\text { Serotonin }(5- \\
\text { HT) } \\
\end{array}$ & $\mathrm{C} 10 \mathrm{H} 12 \mathrm{~N} 2 \mathrm{O}$ & 176.215 & 0.325 \\
\hline Brain & $394 *$ & 102.89 & N/A & N/A & N/A & gamma-Aminobutyric acid & GABA & $\mathrm{C} 4 \mathrm{H} 9 \mathrm{NO} 2$ & 103.12 & 0.23 \\
\hline Heart & 782.5 & 491.39 & $490.89-491.89$ & $\begin{array}{c}\text { Massbank: } 1 \\
\text { mzCloud: } 2 \\
\text { HMDB: } 4 \\
\text { Metlin: } 1475 \\
\end{array}$ & $\begin{array}{l}x \\
\checkmark \\
\checkmark \\
\checkmark \\
\end{array}$ & $\begin{array}{l}\text { Deoxyadenosine } \\
\text { triphosphate }\end{array}$ & dATP & $\mathrm{C} 10 \mathrm{H} 16 \mathrm{~N} 5 \mathrm{O} 12 \mathrm{P} 3$ & 491.182 & -0.208 \\
\hline Heart & 524.5 & 233.39 & $232.89-233.89$ & $\begin{array}{c}\text { Massbank: } 57 \\
\text { mzCloud: } 23 \\
\text { HMDB: } 23 \\
\text { Metlin: >2000 }\end{array}$ & $\begin{array}{l}x \\
x \\
\checkmark \\
\checkmark \\
\end{array}$ & Dopamine 3-O-sulfate & N/A & C8H11NO5S & 233.242 & -0.148 \\
\hline Heart & 402 & 110.89 & $110.39-111.39$ & $\begin{array}{c}\text { Massbank: } 58 \\
\text { mzCloud: } 5 \\
\text { HMDB: } 18 \\
\text { Metlin: } 479 \\
\end{array}$ & $\begin{array}{l}\checkmark \\
\checkmark \\
\checkmark \\
\checkmark\end{array}$ & Histamine & N/A & C5H9N3 & 111.1451 & 0.2551 \\
\hline
\end{tabular}

*Known mass through prior experiments 


\section{Discussion}

The present study sought to determine the viability of MALDI as a procedure to detect neurotransmitters within brain tissue with a high degree of spatial resolution $\left(50 \mu \mathrm{m}^{2}\right)$ through the integration of TPP as a derivatisation agent. Furthermore, the versatility of the procedure was tested to determine whether MALDI can be used on cardiac tissue as well. Finally, the potential to use MALDI as an exploratory procedure to identify unknown compound based on their mass and localization patterns was investigated.

It was found that clear detection of neurotransmitters was possible as dopamine, and serotonin, reference points were detected within $0.325 \mathrm{~g} / \mathrm{mol}$ of their theoretical pyrylium salt mass. Furthermore, GABA was able to be located through the calculation of the GABA- pyrylium salt mass, suggesting the predictive validity of this technique. Using cardiac tissue, MALDI was used to identify compounds without a priori hypotheses: dATP, dopamine 3-O-sulfate, and histamine where characterised based on their mass, and patterns of localisation in cardiac tissue.

Of the four databases used, it should be noted that only HMDB and Metlin were able to identify all compounds based on the pre-derivatisation using the in-built standard parameters and $\mathrm{a} \pm .5 \mathrm{~g} / \mathrm{mol}$ tolerance. Of these databases, Metlin is clearly superior in the number of compounds contained, however, for the purpose of an exploratory design, these data would suggest the use of HMDB initially. HMDB was able to accurately detect all compounds based purely on mass more efficiently that Metlin. If more information about an observed mass is known, the additional criterion may refine the number of results produced by Metlin.

During the exploration of spectra (both brain and cardiac tissue), a masking effect was detected. A high intensity peak at $436 \mathrm{~g} / \mathrm{mol}$, followed by a succession of gradually reducing peaks separated by exactly $1 \mathrm{~g} / \mathrm{mol}$ was present (data not shown). This spacing effect occurred as a result of the presence of carbon-13, a neutral isotope. Thus, we were unable to differentiate between a compound with a mass of $437 \mathrm{~g} / \mathrm{mol}$ and a compound with a mass of $436 \mathrm{~g} / \mathrm{mol}$ in the presence 
of carbon-13. Due to this masking effect, some compounds were unable to be distinguished, in particular acetylcholine $(146.118 \mathrm{~g} / \mathrm{mol})$ and glutamate $(147.053 \mathrm{~g} / \mathrm{mol})$.

Due to TPP specifically reacting with amine groups, this derivatisation agent is not suitable for the detection of other low mass compounds deficient of the amino group. Furthermore, compounds containing two amino groups result in a non-linear change in mass. For example, spermidine $(202.34 \mathrm{~g} / \mathrm{mol})$ contains two amino groups, thus TPP would bind multiple times (418.22 $\mathrm{g} / \mathrm{mol})$ However, due to TPP donating two protons in the case of spermidine, the mass of spermidine would be detected at half of the mass of spermidine with two TPP structures (actual mass; $620.56 \mathrm{~g} / \mathrm{mol}$, mass peak; $310.28 \mathrm{~g} / \mathrm{mol})$. Thus, caution should be taken during compound identification. These caveats are able to be circumvented through the use of alternative derivatisation agents, each of which can be specifically selected to fit the compounds of interest. Furthermore, larger compounds such as lipids, proteins do not require derivatisation. We are currently evaluating the usefulness of MALDI for such larger molecules.

Finally, limited detection was obtained for serotonin and dopamine throughout the tissue sample. These neurotransmitters were clearly detected due to the high intensity obtained at the reference point, and the small tissue localisation. The failure of detecting a clear signal in the brain is likely due to the position of the sagittal section used. This was likely too medial top include the main cell bodies in the midbrain or the majority of the projection sides. Indeed, more lateral sagittal sections show a much stronger presence of dopamine.

\section{Applications}

With some refinement the use of MALDI shows great potential for further elucidating the underlying mechanisms of many psychiatric illness. In particular, the exploratory approach described could allow for the examination of the developmental molecular and neurobiological pathology of early exposure to increased serotonin. As was discussed in the previous chapter, high levels of serotonin during development changes the overall structure of the brain, a phenomenon 
likely to underlie the serotonin paradox. However, given serotonin's broad influence on developmental processes (further complicated by its interaction with 14 receptors subtypes), the functional changes in the brain are likely to be very widespread. The MALDI technique seems ideally suited to identify such widespread changes. Using the subtraction method akin to that used in fMRI studies (Logothetis, 2008), comparisons can be made between the SERT+/+ and SERT ${ }^{-}$ ${ }^{\prime}$ rats.. Moreover, by studying differences at various time point during development would allow us to further elucidate the brain changes underlying the serotonin paradox (Oberlander et al., 2009).

We also demonstrated the ability to use MALDI of cardiac tissue. As discussed in a previous chapter, there is ample evidence that mental disorders such as depression and anxiety disorders also affect the heart. The ability to examine cardiac tissue would allow us to elucidate the mechanisms underlying the association between depression, heart rate variability and cardiovascular disease (Kidwell \& Ellenbroek, 2018b). It is likely that necrohormones and neuromodulators produce molecular and structural alterations in cardiac tissue, which if detected, would inform theory and allow for targeted approaches to understand these mechanisms.

This pilot study sought to determine the viability of MALDI to detect, predict, and identify compounds localised in both brain and cardiac tissue. Within cardiac tissue, dATP, dopamine, 3O-sulfate and histamine were detected, while GABA, dopamine, and serotonin were identified within the brain tissue. These results clearly demonstrated the viability of MALDI in both cardiac tissue and brain tissue without procedural modifications being required between the two tissue modalities. Furthermore, a range of approaches were used (detection, prediction, \& identification), indicating the versatility of MALDI in both hypothesis driven, and exploratory research. 


\section{CHAPTER SEVEN}

Discussion of the Present Findings and Their Contribution

Towards Our Understanding of the SERT Knockout Rat as a

Model of Depressive Disorders 
The preceding chapters have sought to provide further evidence for the use of the SERT Knockout rat as an animal model of major depressive disorder (MDD) in humans. There exists ample evidence implicating a genetic reduction in the serotonin transporter, for instance observed in those expressing the s-allelic version of the 5-HTTLPR, in the aetiology of psychiatric illness, particularly MDD (Collier et al., 1996; Serretti et al., 2002). The SERT knockout rat shows significant neurochemical similarities to humans with this polymorphism, thus prior studies have examined the behavioural and neurobiological similarities between this genetic model and depressed humans. The SERT knockout rat has demonstrated reduced sucrose preference, increased latency in the forced swim test (FST), and anxious traits examined through the elevated plus maze (EPM), home cage emergence, novelty suppressed feeding and open field behaviour (Table 1).

These conventional assays typically reflect a relatively basic method of examining behavioural correlates of highly complex disorders such as MDD or anxiety. It has been suggested that our current generation of animal models of psychiatric illnesses are failing to incorporate the most recent developments in research, a problematic trend which likely underlies the reduction in novel pharmacological treatments demonstrating efficacy in human trials (Ellenbroek and Weiwen, 2018). This trend is unsurprising considering our gold standard animal paradigms are often overly simplistic, and grounded in outdated theory. This is evident both in the analysis of MDD-like symptoms as well as the induction thereof. For example, as discussed in chapter 3, the use of the FST was initially developed in 1977 as a method of detecting potential antidepressant drug effects, evidenced by a reduction in immobility (Porsolt et al., 1977), however, the theory has been "reverse engineered" to the point whereby increased immobility is proposed as being a indicative of increased depression (Castagné, Moser, Roux, \& Porsolt, 2010). Furthermore, with respect to the induction of MDD-like states, as discussed in chapter 1, many of our current animal models of depression are focussed around stress, such as the Chronic Mild Stress (CMS) and social defeat models. While repeated stress undoubtedly contributes to the pathogenesis of MDD, and these models typically 
show behavioural and neurobiological characteristics indicative of MDD (Willner, 2005), these abnormalities are often transient in nature, and, most importantly do not take into consideration the substantial genetic contribution to the aetiology of MDD.

Throughout the previous chapters we have attempted to provide additional evidence through the examination of behavioural, neurobiological, and physiological characteristics of SERT knock out rats using more sophisticated measures. The measures used were selected to more closely mirror the effects of MDD observed in humans, while incorporating a genetic component, the observed are unlikely be transient in nature. The major findings will be summarised (See table 1):

Social anhedonia, often seen in depressed humans, (Nezlek et al., 1994), was examined through social conditioned place preference. Preference coefficients revealed the development of preference for the paired chamber in $\mathrm{SERT}^{+/+}$animals, but not $\mathrm{SERT}^{+/}$or $\mathrm{SERT}^{-/}$groups. Furthermore, genotypes did not differ in their play related interactions during these trials, while an increase in non-play social interaction was observed in SERT ${ }^{-/}$animals. These interactions were not significantly associated with preference coefficients, thus could not explain the lack of preference development. These results suggest that $\mathrm{SERT}^{+/-}$, and $\mathrm{SERT}^{-/}$animals experience social anhedonia, thus mirroring the anhedonia previously reported in sucrose drinking (see table 1).

Anxiety disorders show significant associations with MDD, whereby high comorbidity is observed, and a significant overlap in aetiology is present (Kessler et al., 1996). As such, the presence of anxiety traits are likely to be observed in animal models of depression. Moreover, genetic reductions in SERT activity in humans also contribute to the aetiology of anxiety disorders. Indeed, conventional measures of anxiety have demonstrated that $\mathrm{SERT}^{-/-}$rats show significantly increased anxiety like behaviour (J. Olivier et al., 2008). As mentioned above, many of the animal models only induce transient changes in behaviour, and even in the SERT ${ }^{-/}$rats, changes in 
anxiety, while assessed in multiple paradigms, have only been assessed at a single time point. The reason, as discussed in chapter 3 is that virtually all of the anxiety tests suffer from the one time tolerance (OT'T) effect and thus do not allow repeated assessments of anxiety (File et al., 1990). Therefore, we set out to examine these behaviours through a modified version of a relatively novel measure of anxiety in animals in chapter 2. Firstly, we demonstrated the SERT ${ }^{-/}$animals show a significant increase in anxious behaviour through multiple measures, which was found to persist over the course of the experiment, thus circumventing the OT'T effect. Secondly, we demonstrated that the behaviours exhibited during each trial are reflective of the same construct; anxiety. Finally, we were able to demonstrate a novel coding measure of retrieval behaviour to detect differences between all three genotypes, suggesting a greater sensitivity to intermediate levels of anxiety.

There exists a strong association between MDD and cardiovascular disease (CVD), a relationship likely to be mediated through alterations in heart rate variability (HRV) (Kidwell \& Ellenbroek, 2018b). Furthermore, a bidirectional relationship between HRV and MDD likely exists whereby, MDD is predictive of reduced HRV, while reductions in HRV have been shown to predict the onset of MDD. Given these associations, and the ability to examine HRV in humans and rats through near identical measures, in chapter 3 we sought to investigate HRV in SERT knockout animals, thus establishing a novel biomarker of depression. Through the integration of a novel surgical technique, 24 hours of ECG data were collected. However, due to the unforeseen impact of post-operative treatments, the observed data contained significant amounts of intercohort variance. Following this, several methods were used to isolate the origin of the variance. The variance observed was most prevalent within the HF percentage parameter, implicating sinoatrial depolarisation, a finding further supported by the flattened p-wave morphology, indicative of hyperkalaemia. Through splitting of cohorts, trends began to emerge, suggesting a likely reduction in $\mathrm{HRV}$ in the $\mathrm{SERT}^{-/}$group which warrants further investigation. 
The neurobiological mechanisms underlying MDD are yet to be fully understood. Moreover, as mentioned in the introduction, serotonin appears to play a paradoxical effect in MDD (anxiety disorders): As evidenced by the literature and in the present paper, genetic reductions in the SERT enhances symptoms of MDD and anxiety, while pharmacological reductions reduce such symptoms. While many proposed mechanisms have been demonstrated, there is a large literature base to suggest numerous factors during early development result in the predisposition to the development of MDD (Wankerl et al., 2014). In line with this, serotonin has been shown to be intimately involved in many aspects of neurodevelopment. For example, Kraus et al., (2017) reviewed this complex relationship between serotonin and neuroplasticity demonstrating not only direct, but an intricate system of indirect effects. For example, the $5-\mathrm{HT}^{1 \mathrm{~A}}$ receptor has been shown to influence neurogenesis, neuroprotection, astroglia, and dendritic maturation, however, these interactions were mediated through eight distinct mechanisms including brain derived neurotropic factor $(\mathrm{BDNF})$, protein kinase $\mathrm{B}$, and the cAMP responsiveelement binding (CREB). Furthermore, $5-\mathrm{HT}^{4}$ was shown to have an effect of neurogenesis, spine morphology and synaptic plasticity, effects mediated through six mechanisms including long term potentiation/depression, BDNF, and extracellular signal-regulated kinases. This demonstrates the complex and diverse role of serotonin within the specific process of neuroplasticity, while equally complex roles exist in other processes (e.g. Rosen, 2009).

In addition, serotonin is involved in adult neuroplasticity. For instance, a reduction in neurogenesis has been observed in depressed adults, an effect that has been shown to be normalised following treatment with serotonin transporter blockers (Anacker et al., 2011), implicating the role of neurogenesis in the aetiology of MDD. To investigate the developmental alterations that occur as a result of increased exposure to serotonin, we set out to examine neurogenesis in the dentate gyrus region of the hippocampus on $\mathrm{SERT}^{+/+}, \mathrm{SERT}^{+/}$, and $\mathrm{SERT}^{-/-}$ animals (PND7). Using BrdU immunohistochemistry, a significant increase in neurogenesis was observed in SERT $\mathrm{S}^{-/}$animals. 
Given the complexity of serotonin's actions on neurodevelopment, it is virtually impossible to predict how the brain changes in the presence of high extracellular concentrations of serotonin during the critical developmental period. This thus requires a more global, hypothesis free method. Therefore, in chapter 6 we aimed to investigate whether MALDI would provide such an approach. While only meant as a proof-of-concept chapter, the results suggest that MALDI is sensitive enough to detect neurotransmitter levels in the brain. Moreover, it has a good spatial resolution to be able to identify regional differences. Finally, the method is accurate enough to identify unknown compounds, as evidenced by the analysis of cardiac tissue.

Table 1 Summary of known similarities and dissimilarities

\begin{tabular}{|c|c|c|c|}
\hline Category & Human & SERT knockout & \\
\hline Depressed mood & Diagnosic Criteria & $\begin{array}{c}\mathrm{N} / \mathrm{A} \\
\text { (USVs imply negative internal state) }\end{array}$ & Chapter 3 \\
\hline Feelings of worthlessness & Diagnosic Criteria & N/A & N/A \\
\hline Anhedonia & Diagnosic Criteria & $\begin{array}{c}\text { Yes } \\
\text { general and social }\end{array}$ & Chapter 2 \\
\hline Weight change & Diagnosic Criteria & $\begin{array}{c}\text { Yes } \\
\text { Developmental delay }\end{array}$ & NA \\
\hline Sleep dysfunction & $\begin{array}{c}\text { Diagnosic Criteria } \\
\text { (hypersomnia or hyposomnia) }\end{array}$ & Increased REM & Wisor et al., (2003) \\
\hline Psychomotor & $\begin{array}{c}\text { Diagnosic Criteria } \\
\text { (agitation or retardation) }\end{array}$ & No & N/A \\
\hline Fatigue & Diagnosic Criteria & N/A & N/A \\
\hline Diminished cognition & Diagnosic Criteria & Yes & Olivier et al., (2008) \\
\hline Thoughts of death & Diagnosic Criteria & N/A & N/A \\
\hline Persistent effects & Diagnosic Criteria & Yes & All \\
\hline Comornid anxiety & Yes & Yes & Chapter 3 \\
\hline Comorbid Drug misuse & Yes & Yes & Oakly et al. (2014) \\
\hline HRV & Yes & Probable & Chapter 4 \\
\hline Cardiovascular pathology & Yes & Yes & Chapter 4 \\
\hline Altered Neurogenesis & Yes & Yes & $\begin{array}{c}\text { Chapter } 5 \text { / Schipper et } \\
\text { al., (2011) }\end{array}$ \\
\hline Altered respose to emotional stimui & Yes & Yes & Bylsma et al., (2008) \\
\hline Amygdala hyperactivity & Yes & Yes & Bylsma et al., (2008) \\
\hline Sexual dysfunction & Yes & Yes & Kennedy et al., (1999) \\
\hline Developmental factors & Yes & Yes & Chapter 5 \\
\hline Serotonin receptor downregulation & Yes & Yes & Stahl et al., (1994) \\
\hline Reduced BDNF & Yes & Yes & Shmizu et al., (2003) \\
\hline HPA Dysregulation & Yes & Yes & Bylsma et al., (2008) \\
\hline
\end{tabular}


Mechanisms underlying the behavioural, neurobiological, and physiological abnormalities of the SERT

\section{knockout animal}

Throughout the prior chapters we have demonstrated significant behavioural, neurobiological, and physiological alterations in response to the genetic reduction of serotonin transported expression. Most importantly, these alterations show a great degree of similarity to the deficits observed in depressed humans, particularly in regard to the SERT ${ }^{-/-}$animals. The broad impact of this serotonergic modulation highlights the complexity of the interactions underlying the development and maintenance of MDD, and the models by which we seek to mirror it. Clearly more research is needed to begin to identify the neurobiological underpinnings underlying the association between enhanced serotonin during development and the symptoms of MDD, however, our current understanding allows for informed theories to be developed. For instance the downregulation of the $5-\mathrm{HT}^{1 \mathrm{~A}}$ receptor subtype has frequently been reported in depressed humans, an effect which has further been demonstrated in the $\mathrm{SERT}^{-/}$rats, but not $\mathrm{SERT}^{+/-}$ animals (Olivier et al., 2007). Importantly, this downregulation likely underlies the social preference deficits in the SERT knockout rats. As demonstrated by Dunn et al. (1989), administration of selective 5-HT ${ }^{1 \mathrm{~A}}$ agonists; buspirone, fepirone, and 8-hydroxy-2-(di-n-propylamino) tetralin (8OH-DPAT) all produced significant increases in social interaction. Furthermore, using CPP, intraperitoneal administration and direct microinjections into the raphe-nuclei of 8-OH-DPAT were sufficient in producing a significant preference, thus implicating $5-\mathrm{HT}^{1 \mathrm{~A}}$ agonism in social interaction and reward. The impact of the $5-\mathrm{HT}^{1 \mathrm{~A}}$ receptor is not limited to social interaction and reward, rather the $5-\mathrm{HT}^{1 \mathrm{~A}}$ receptor has been implicated in anxiety, whereby the anxiolytic effects of fluvoxamine are blocked through $5-\mathrm{HT}^{1 \mathrm{~A}}$ antagonism, implying the anxiolytic effects of fluvoxamine are mediated by the $5-\mathrm{HT}^{1 \mathrm{~A}}$ receptor (Ichimaru, Egawa, \& Sawa, 1995). Intriguingly, the effects of the $5-\mathrm{HT}^{1 \mathrm{~A}}$ receptor extend beyond behavioural observations, as reductions of the 5-HT ${ }^{1 \mathrm{~A}}$ receptor has been demonstrated to produce significant cardiovascular effects which would produce reductions in the parasympathetic components of HRV (Jordan, 2005), while 5-HT ${ }^{1 \mathrm{~A}}$ 
stimulation improves vagal control (Sporton, Shepheard, Jordan, \& Ramage, 1991). Finally, stimulation of $5-\mathrm{HT}^{1 \mathrm{~A}}$ receptors enhance dentate gyrus neurogenesis (Samuels, \& Hen, 2011).

While these data clearly point to a potential role of reduced $5-\mathrm{HT}^{1 \mathrm{~A}}$ receptors in the phenotype of the $\mathrm{SERT}^{-/-}$rats, it should be kept in mind that the effects are likely much more complex. Unpublished data from our own lab group have demonstrated a reduction in 5 -HT ${ }^{2 \mathrm{C}}$ activation in the $\mathrm{SERT}^{-/}$animals, suggesting the diminished serotonin receptor activation may not be limited to these receptor subtypes, but likely impacts most if not all the 14 known subtypes. If this proposition holds true, due to the wide variety of functions influenced through the 5-HT receptors (for a full review see Gaspar et al. (2003) and see also above), these downregulatory effects likely contribute to the complex neuropathology of MDD and the SERT knockout line of rats.

For example in addition to the $5-\mathrm{HT}^{1 \mathrm{~A}}$ receptor, stimulation of the $5-\mathrm{HT}^{1 \mathrm{~B}}$ or $5-\mathrm{HT}^{2 \mathrm{C}}$ receptor also enhances (adult) neurogenesis (Banasr, Hery, Printemps, and Daszuta (2004). The authors further demonstrated that blockade of the $5-\mathrm{HT}^{2 \mathrm{C}}$ receptor reduced neurogenesis. Serotonin receptors also have a complex effect of cardiovascular function. For example the 5$\mathrm{HT}^{2 \mathrm{~B}}$ produces excitatory effects, while the opposite is true for the $5-\mathrm{HT}^{2 \mathrm{C}}$ (Jordan (2005). Furthermore, $5-\mathrm{HT}^{7}$ agonism produced inhibitory effects while $5-\mathrm{HT}^{3}$ agonism resulted in excitation.

An interesting aspect of the our genetic model is the downregulatory dissociation between the SERT ${ }^{+/-}$and SERT ${ }^{-/}$animals (J. D. Olivier et al., 2008), with SERT ${ }^{-/-}$but not $\mathrm{SERT}^{+/-}$rats having reduced $5-\mathrm{HT}^{1 \mathrm{~A}}$ receptor activity. This may explain a number of the effects observed prior chapters, whereby the SERT ${ }^{-/}$animals frequently demonstrated significantly altered behaviours and biological effects, while the SERT ${ }^{+/-}$animals failed to reach significance. While in the $\mathrm{SERT}^{+/-}$ animals there is an increased extracellular serotonin, this increase may not be sufficient enough to produce the downregulation, thus often fails to exhibit the deficits observed in the $\mathrm{SERT}^{-/}$ animals. It should be noted that while often not reaching significance, a number of chapters 
demonstrate a trend suggesting a minor deficit may be present. For example, in chapter 1, the $\mathrm{SERT}^{+/-}$animals did not demonstrate the development of preference like the SERT ${ }^{+/+}$group, however, SERT ${ }^{+/-}$groups did not significantly differ from either $\mathrm{SERT}^{+/+}$, nor $\mathrm{SERT}^{-/-}$animals, thus implying an intermediate effect. Furthermore, in chapter 3, all groups differed in retrieval scores, which, along with the absence of preference development suggest effects outside of the serotonergic system to be impacting the behaviours. Moreover, it should be kept in mind that, so far, the vast majority of studies (including the studies that observed a downregulation in $5-\mathrm{HT}^{1 \mathrm{~A}}$ and $5-\mathrm{HT}^{2 \mathrm{C}}$ receptors) have been performed in adulthood. Given the dynamicity of the brain, more studies investigating young animals is urgently required.

\section{Translatability of the SERT Knockout}

The data presented throughout the prior chapters identified several similarities between the SERT knockout rat and depressed humans, thus providing a high degree of translatable validity to the SERT knockout rat as an animal model of depression and anxiety. This model should be strongly considered when developing novel pharmacological treatments of MDD, as the demonstrated effects closely mirror the human aetiology (See table 1). One significant effect which has likely limited the use of the SERT knockout rat in the development on novel treatments is the lack of predictive validity, whereby SERT knockouts do not response to SSRIs. It should be noted that response to MAOIs and SNRIs have been reported (Haenisch \& Bönisch, 2011), thus predictive validity is not absent. This is problematic, as while we understand the general mechanisms by which SSRIs exhibit their effects, the precise mechanisms underlying their antidepressant efficacy are yet to be fully understood (Kupfer, Frank, \& Phillips, 2012). Therefore, the failed response to them does not automatically imply poor model, rather highlights the need for novel treatments outside of the current serotonin transporter focused treatments. To support this, the efficacy of our current generation has been questioned (Kirsch et al., 2008), while response to treatment is known to be delayed (Uher et al., 2011). More troubling, one third of those with depression fail to respond to current first line treatments (Souery, Papakostas, \& Trivedi, 2006), 
an effect which has been shown to be mediated by the 5-HTTLPR polymorphism (Smeraldi et al., 1998). Finally, treatments outside of the use of SSRIs, such as vagal nerve stimulation, have demonstrated high efficacy, and the ability to treat those previously classed as treatment resistant (Nemeroff et al., 2006). The major limitation of the SERT ${ }^{-/-}$rat as a model of the human condition is the complete reduction of serotonin transporter expression, whereas humans expressing the $\mathrm{s}$ allelic form of the 5-HTTLPR polymorphism show only a partial reduction (Gotlib, Joormann, Minor \& Hallmayer, 2008). This is likely to explain the gene x environment interaction observed in humans where a stressor is required to produce the onset of a depressive episode, while the SERT $^{-/-}$animals demonstrate abnormal characteristic in the absence of a stressor (Olivier et al., 2008). A more suitable model of the MDD, would be the $\mathrm{SERT}^{+/-}$rat, whereby in the absence of a stressor, do not differ from the SERT ${ }^{+/+}$rat (as seen in chapters 3, 4, \& 5), whereas when exposed to stress, begin to exhibit dysfunctional behaviours (see chapter 2). This suggests that to provide greater face validity, stress induction such as CMS should be used with $\mathrm{SERT}^{+/-}$rats, likely producing the desired phenotype to emerge. Furthermore, our current behavioural assays may not be sufficiently sensitive to detect these dysfunctions in their current state, however, more sensitive behavioural measures as seen in retrieval scores (chapter 3). Physiological measures such as HRV have demonstrated greater sensitivity than behavioural measures (Grippo et al., 2003), thus should be incorporated in future models of MDD. This highlights a strong candidate for the development of novel treatments of MDD, whereby pharmacological stimulation of the parasympathetic nervous system warrants further attention. 


\section{References}

Acharya, U. R., Joseph, K. P., Kannathal, N., Lim, C. M., \& Suri, J. S. (2006). Heart rate variability: a review. Medical and biological engineering and computing, 44(12), 1031-1051.

Agorastos, A., Kellner, M., Stiedl, O., Muhtz, C., Becktepe, J. S., Wiedemann, K., \& Demiralay, C.

(2014). The 5-HTTLPR genotype modulates heart rate variability and its adjustment by

pharmacological panic challenge in healthy men. Journal of psychiatric research, 50, 51-58.

Akimova, E., Lanzenberger, R., \& Kasper, S. (2009). The serotonin-1A receptor in anxiety disorders. Biological psychiatry, 66(7), 627-635.

Alderfer, C. P. (1969). An empirical test of a new theory of human needs. Organizational behavior and human performance, 4(2), 142-175.

Alexander, R. D. (1974). The evolution of social behavior. Annual review of ecology and systematics, 5(1), 325-383.

Anacker, C., Zunszain, P. A., Cattaneo, A., Carvalho, L. A., Garabedian, M. J., Thuret, S., . . Pariante,

C. M. (2011). Antidepressants increase human hippocampal neurogenesis by activating the glucocorticoid receptor. Molecular Psychiatry, 16(7), 738.

Ansorge, M. S., Zhou, M., Lira, A., Hen, R., \& Gingrich, J. A. (2004). Early-life blockade of the 5-HT transporter alters emotional behavior in adult mice. Science, 306(5697), 879-881.

Appelhans, B. M., \& Luecken, L. J. (2006). Heart rate variability as an index of regulated emotional responding. Review of general psychology, 10(3), 229.

Aromaa, A., Raitasalo, R., Reunanen, A., Impivaara, O., Heliovaara, M., Knekt, P., . . Maatela, J. (1994). Depression and cardiovascular diseases. Acta Psychiatrica Scandinavica, 89(s377), 77-82.

Association, A. P. (2013). Diagnostic and statistical manual of mental disorders (DSM-5 ${ }^{\circledR}$ ): American Psychiatric Pub.

Balzarotti, S., Biassoni, F., Colombo, B., \& Ciceri, M. (2017). Cardiac Vagal Control as a Marker of Emotion Regulation in Healthy Adults: A Review. Biological psychology.

Banasr, M., \& Duman, R. S. (2007). Regulation of neurogenesis and gliogenesis by stress and antidepressant treatment. CNS \& Neurological Disorders-Drug Targets (Formerly Current Drug Targets-CNS \& Neurological Disorders), 6(5), 311-320.

Banasr, M., Hery, M., Printemps, R., \& Daszuta, A. (2004). Serotonin-induced increases in adult cell proliferation and neurogenesis are mediated through different and common 5-HT receptor subtypes in the dentate gyrus and the subventricular zone. Neuropsychopharmacology, 29(3), 450.

Barnes, N. M., \& Sharp, T. (1999). A review of central 5-HT receptors and their function. Neuropharmacology, 38(8), 1083-1152.

Barr, C. S., Newman, T. K., Schwandt, M., Shannon, C., Dvoskin, R. L., Lindell, S. G., . . Lesch, K. P. (2004). Sexual dichotomy of an interaction between early adversity and the serotonin transporter gene promoter variant in rhesus macaques. Proceedings of the National Academy of Sciences, 101(33), 12358-12363.

Baumeister, R. F. (1990). Suicide as escape from self. Psychological review, 97(1), 90.

Beck, A. T., Steer, R. A., \& Brown, G. K. (1996). Beck depression inventory-II. San Antonio, 78(2), 490498.

Becker, C., Zeau, B., Rivat, C., Blugeot, A., Hamon, M., \& Benoliel, J. (2008). Repeated social defeatinduced depression-like behavioral and biological alterations in rats: involvement of cholecystokinin. Molecular Psychiatry, 13(12), 1079.

Berman, R. M., Narasimhan, M., \& Charney, D. S. (1997). Treatment-refractory depression: Definitions and characteristics. Depression and anxiety, 5(4), 154-164.

Bhardwaj, R. D., Curtis, M. A., Spalding, K. L., Buchholz, B. A., Fink, D., Björk-Eriksson, T., . . Eriksson,

P. S. (2006). Neocortical neurogenesis in humans is restricted to development. Proceedings of the National Academy of Sciences, 103(33), 12564-12568. 
Blumenthal, J. A., Williams, S. R., Wallace, A. G., Williams Jr, R. B., \& Needles, T. L. (1982).

Physiological and psychological variables predict compliance to prescribed exercise therapy in patients recovering from myocardial infarction. Psychosomatic medicine, 44(6), 519-527.

Boettger, S., Hoyer, D., Falkenhahn, K., Kaatz, M., Yeragani, V. K., \& Bär, K.-J. (2006). Altered diurnal autonomic variation and reduced vagal information flow in acute schizophrenia. Clinical Neurophysiology, 117(12), 2715-2722.

Bond, A. J. (2005). Antidepressant treatments and human aggression. European journal of pharmacology, 526(1-3), 218-225.

Bonnin, A., Torii, M., Wang, L., Rakic, P., \& Levitt, P. (2007). Serotonin modulates the response of embryonic thalamocortical axons to netrin-1. Nature neuroscience, 10(5), 588.

Borsini, F., Volterra, G., \& Meli, A. (1986). Does the behavioral "despair" test measure "despair"? Physiology \& behavior, 38(3), 385-386.

Boulos, Z., Rosenwasser, A. M., \& Terman, M. (1980). Feeding schedules and the circadian organization of behavior in the rat. Behavioural brain research, 1(1), 39-65.

Brenes, J. C., Rodríguez, O., \& Fornaguera, J. (2008). Differential effect of environment enrichment and social isolation on depressive-like behavior, spontaneous activity and serotonin and norepinephrine concentration in prefrontal cortex and ventral striatum. Pharmacology Biochemistry and Behavior, 89(1), 85-93.

Brezun, J., \& Daszuta, A. (1999). Depletion in serotonin decreases neurogenesis in the dentate gyrus and the subventricular zone of adult rats. Neuroscience, 89(4), 999-1002.

Brothers, L. (1996). Brain mechanisms of social cognition. Journal of psychopharmacology, 10(1), 2-8.

Burgdorf, J., Panksepp, J., \& Moskal, J. R. (2011). Frequency-modulated 50 kHz ultrasonic vocalizations: a tool for uncovering the molecular substrates of positive affect. Neuroscience \& Biobehavioral Reviews, 35(9), 1831-1836.

Cacioppo, J. T., Hawkley, L. C., \& Thisted, R. A. (2010). Perceived social isolation makes me sad: 5 year cross-lagged analyses of loneliness and depressive symptomatology in the Chicago Health, Aging, and Social Relations Study. Psychology and aging, 25(2), 453.

Canli, T., \& Lesch, K.-P. (2007). Long story short: the serotonin transporter in emotion regulation and social cognition. Nature neuroscience, 10(9), 1103.

Cannon, W. B. (1911). The mechanical factors of digestion: Longmans, Green \& Company.

Cardiology, T. F. o. t. E. S. o. (1996). Heart rate variability, standards of measurement, physiological interpretation, and clinical use. circulation, 93, 1043-1065.

Carnevali, L., \& Sgoifo, A. (2014). Vagal modulation of resting heart rate in rats: the role of stress, psychosocial factors, and physical exercise. Frontiers in physiology, 5, 118.

Carney, R. M., Rich, M. W., Tevelde, A., Saini, J., Clark, K., \& Jaffe, A. S. (1987). Major depressive disorder in coronary artery disease. The American journal of cardiology, 60(16), 1273-1275.

Carobrez, A., \& Bertoglio, L. (2005). Ethological and temporal analyses of anxiety-like behavior: the elevated plus-maze model 20 years on. Neuroscience \& Biobehavioral Reviews, 29(8), 11931205.

Carola, V., Frazzetto, G., Pascucci, T., Audero, E., Puglisi-Allegra, S., Cabib, S., . . Gross, C. (2008). Identifying molecular substrates in a mouse model of the serotonin transporterx environment risk factor for anxiety and depression. Biological psychiatry, 63(9), 840-846.

Carrillo, M., Ricci, L. A., Coppersmith, G. A., \& Melloni, R. H. (2009). The effect of increased serotonergic neurotransmission on aggression: a critical meta-analytical review of preclinical studies. Psychopharmacology, 205(3), 349-368.

Casanova, M. F., Hensley, M. K., Sokhadze, E. M., El-Baz, A. S., Wang, Y., Li, X., \& Sears, L. (2014). Effects of weekly low-frequency rTMS on autonomic measures in children with autism spectrum disorder. Frontiers in human neuroscience, 8, 851.

Cases, O., Vitalis, T., Seif, I., De Maeyer, E., Sotelo, C., \& Gaspar, P. (1996). Lack of barrels in the somatosensory cortex of monoamine oxidase A-deficient mice: role of a serotonin excess during the critical period. Neuron, 16(2), 297-307. 
Caspi, A., \& Moffitt, T. E. (2006). Gene-environment interactions in psychiatry: joining forces with neuroscience. Nature Reviews Neuroscience, 7(7), 583.

Caspi, A., Sugden, K., Moffitt, T. E., Taylor, A., Craig, I. W., Harrington, H., . . Braithwaite, A. (2003). Influence of life stress on depression: moderation by a polymorphism in the 5-HTT gene. Science, 301(5631), 386-389.

Castagné, V., Moser, P., Roux, S., \& Porsolt, R. D. (2010). Rodent models of depression: forced swim and tail suspension behavioral despair tests in rats and mice. Current Protocols in Pharmacology, 49(1), 5.8. 1-5.8. 14.

Cheng, Y., Hogarth, K. A., O'Sullivan, M. L., Regnier, M., \& Pyle, W. G. (2015). 2-Deoxyadenosine triphosphate restores the contractile function of cardiac myofibril from adult dogs with naturally occurring dilated cardiomyopathy. American Journal of Physiology-Heart and Circulatory Physiology, 310(1), H80-H91.

Cole, D. A., Peeke, L. G., Martin, J. M., Truglio, R., \& Seroczynski, A. (1998). A longitudinal look at the relation between depression and anxiety in children and adolescents. Journal of consulting and clinical psychology, 66(3), 451.

Collier, D., Stöber, G., Li, T., Heils, A., Catalano, M., Di, D. B., . . Bengel, D. (1996). A novel functional polymorphism within the promoter of the serotonin transporter gene: possible role in susceptibility to affective disorders. Molecular Psychiatry, 1(6), 453-460.

Cook, M. N., Crounse, M., \& Flaherty, L. (2002). Anxiety in the elevated zero-maze is augmented in mice after repeated daily exposure. Behavior genetics, 32(2), 113-118.

Crişan, L. G., Pană, S., Vulturar, R., Heilman, R. M., Szekely, R., Drugă, B., . . Miu, A. C. (2009).

Genetic contributions of the serotonin transporter to social learning of fear and economic decision making. Social cognitive and affective neuroscience, 4(4), 399-408.

Cryan, J. F., \& Sweeney, F. F. (2011). The age of anxiety: role of animal models of anxiolytic action in drug discovery. British journal of pharmacology, 164(4), 1129-1161.

Cuthbert, B. N. (2014). The RDoC framework: facilitating transition from ICD/DSM to dimensional approaches that integrate neuroscience and psychopathology. World Psychiatry, 13(1), 2835.

de Jong, J. G., van der Vegt, B. J., Buwalda, B., \& Koolhaas, J. M. (2005). Social environment determines the long-term effects of social defeat. Physiology \& behavior, 84(1), 87-95.

Deacon, R. M. (2013). The successive alleys test of anxiety in mice and rats. Journal of visualized experiments: JoVE(76).

Denk, F., Walton, M., Jennings, K., Sharp, T., Rushworth, M., \& Bannerman, D. (2005). Differential involvement of serotonin and dopamine systems in cost-benefit decisions about delay or effort. Psychopharmacology, 179(3), 587-596.

Djavadian, R. L. (2004). Serotonin and neurogenesis in the hippocampal dentate gyrus of adult mammals. Acta neurobiologiae experimentalis, 64(2), 189-202.

Douglas, L. A., Varlinskaya, E. I., \& Spear, L. P. (2004). Rewarding properties of social interactions in adolescent and adult male and female rats: impact of social versus isolate housing of subjects and partners. Developmental psychobiology, 45(3), 153-162.

Drevets, W. C., Frank, E., Price, J. C., Kupfer, D. J., Holt, D., Greer, P. J., ... Mathis, C. (1999). PET imaging of serotonin $1 \mathrm{~A}$ receptor binding in depression. Biological psychiatry, 46(10), 13751387.

Duke, A. A., Bègue, L., Bell, R., \& Eisenlohr-Moul, T. (2013). Revisiting the serotonin-aggression relation in humans: A meta-analysis. Psychological bulletin, 139(5), 1148.

Duman, R. S., Malberg, J., Nakagawa, S., \& D'Sa, C. (2000). Neuronal plasticity and survival in mood disorders. Biological psychiatry, 48(8), 732-739.

Duman, R. S., Malberg, J., \& Thome, J. (1999). Neural plasticity to stress and antidepressant treatment. Biological psychiatry, 46(9), 1181-1191. 
Dunn, R. W., Corbett, R., \& Fielding, S. (1989). Effects of 5-HT1A receptor agonists and NMDA receptor antagonists in the social interaction test and the elevated plus maze. European journal of pharmacology, 169(1), 1-10.

Einon, D. F., \& Morgan, M. (1977). A critical period for social isolation in the rat. Developmental Psychobiology: The Journal of the International Society for Developmental Psychobiology, 10(2), 123-132.

Ellenbogen, M. A., Young, S. N., Dean, P., Palmour, R. M., \& Benkelfat, C. (1996). Mood response to acute tryptophan depletion in healthy volunteers: sex differences and temporal stability. Neuropsychopharmacology, 15(5), 465.

Ellenbroek, B. A., August, C., \& Youn, J. (2016). Does prenatal valproate interact with a genetic reduction in the serotonin transporter? A rat study on anxiety and cognition. Frontiers in neuroscience, 10, 424.

Ellenbroek, B. A., \& Sengul, H. K. (2017). Autism spectrum disorders: Autonomic alterations with a special focus on the heart. Heart and Mind, 1(2), 78.

Endo, K., Ando, S., Shimodera, S., Yamasaki, S., Usami, S., Okazaki, Y., . . Nishida, A. (2017). Preference for solitude, social isolation, suicidal ideation, and self-harm in adolescents. Journal of Adolescent Health, 61(2), 187-191.

Eriksson, P. S., Perfilieva, E., Björk-Eriksson, T., Alborn, A.-M., Nordborg, C., Peterson, D. A., \& Gage, F. H. (1998). Neurogenesis in the adult human hippocampus. Nature medicine, 4(11), 1313.

Erlinge, S., Göransson, G., Hansson, L., Högstedt, G., Liberg, O., Nilsson, I. N., . . . Sylvén, M. (1983). Predation as a regulating factor on small rodent populations in southern Sweden. Oikos, 3652.

Ettinger, P. O., Regan, T. J., \& Oldewurtel, H. A. (1974). Hyperkalemia, cardiac conduction, and the electrocardiogram: a review. American heart journal, 88(3), 360-371.

Everson, S. A., Goldberg, D. E., Kaplan, G. A., Cohen, R. D., Pukkala, E., Tuomilehto, J., \& Salonen, J. T. (1996). Hopelessness and risk of mortality and incidence of myocardial infarction and cancer. Psychosomatic medicine, 58(2), 113-121.

Faes, L., Chon, K. H., \& Nollo, G. (2009). A method for the time-varying nonlinear prediction of complex nonstationary biomedical signals. IEEE transactions on biomedical engineering, 56(2), 205-209.

Fanous, S., Hammer Jr, R. P., \& Nikulina, E. M. (2010). Short-and long-term effects of intermittent social defeat stress on brain-derived neurotrophic factor expression in mesocorticolimbic brain regions. Neuroscience, 167(3), 598-607.

Fava, M., Rankin, M. A., Wright, E. C., Alpert, J. E., Nierenberg, A. A., Pava, J., \& Rosenbaum, J. F. (2000). Anxiety disorders in major depression. Comprehensive Psychiatry, 41(2), 97-102.

Fergusson, D., Doucette, S., Glass, K. C., Shapiro, S., Healy, D., Hebert, P., \& Hutton, B. (2005). Association between suicide attempts and selective serotonin reuptake inhibitors: systematic review of randomised controlled trials. Bmj, 330(7488), 396.

File, S. E., Lippa, A. S., Beer, B., \& Lippa, M. T. (2004). Animal tests of anxiety. Current protocols in neuroscience, 26(1), 8.3. 1-8.3. 22.

File, S. E., Mabbutt, P. S., \& Hitchcott, P. K. (1990). Characterisation of the phenomenon of "one-trial tolerance" to the anxiolytic effect of chlordiazepoxide in the elevated plus-maze. Psychopharmacology, 102(1), 98-101.

Finlayson, K., Lampe, J. F., Hintze, S., Würbel, H., \& Melotti, L. (2016). Facial indicators of positive emotions in rats. PloS one, 11(11), e0166446.

Fox, M. A., Stein, A. R., French, H. T., \& Murphy, D. L. (2010). Functional interactions between 5HT2A and presynaptic 5-HT1A receptor-based responses in mice genetically deficient in the serotonin 5-HT transporter (SERT). British journal of pharmacology, 159(4), 879-887.

Gaspar, P., Cases, O., \& Maroteaux, L. (2003). The developmental role of serotonin: news from mouse molecular genetics. Nature Reviews Neuroscience, 4(12), 1002.

Goldberg, D. P. (2014). Anxious forms of depression. Depression and anxiety, 31(4), 344-351. 
Gonzalez, M. B., Snyderman, T. B., Colket, J. T., Arias, R. M., Jiang, J. W., O'Connor, C. M., \& Krishnan, K. R. (1996). Depression in patients with coronary artery disease. Depression, 4(2), 57-62. Gould, E., \& Tanapat, P. (1999). Stress and hippocampal neurogenesis. Biological psychiatry, 46(11), 1472-1479.

Grippo, A. J., Beltz, T. G., \& Johnson, A. K. (2003). Behavioral and cardiovascular changes in the chronic mild stress model of depression. Physiology \& behavior, 78(4-5), 703-710.

Haenisch, B., \& Bönisch, H. (2011). Depression and antidepressants: insights from knockout of dopamine, serotonin or noradrenaline re-uptake transporters. Pharmacology \& therapeutics, 129(3), 352-368.

Hall, H., Lundkvist, C., Halldin, C., Farde, L., Pike, V., McCarron, J., . . Wikström, H. (1997). Autoradiographic localization of 5-HT1A receptors in the post-mortem human brain using

[3H] WAY-100635 and [11C] way-100635. Brain Research, 745(1-2), 96-108.

Hamilton, M. (1960). A rating scale for depression. Journal of neurology, neurosurgery, and psychiatry, 23(1), 56.

Hånell, A., \& Marklund, N. (2014). Structured evaluation of rodent behavioral tests used in drug discovery research. Frontiers in behavioral neuroscience, 8, 252.

Hariri, A. R., Mattay, V. S., Tessitore, A., Kolachana, B., Fera, F., Goldman, D., . . Weinberger, D. R. (2002). Serotonin transporter genetic variation and the response of the human amygdala. Science, 297(5580), 400-403.

Harmer, C., Bhagwagar, Z., Perrett, D., Völlm, B., Cowen, P., \& Goodwin, G. (2003). Acute SSRI administration affects the processing of social cues in healthy volunteers.

Neuropsychopharmacology, 28(1), 148.

Hay, S. I., Jayaraman, S. P., Truelsen, T., Sorensen, R. J., Millear, A., Giussani, G., \& Beghi, E. (2017). GBD 2015 disease and injury incidence and prevalence collaborators. Global, regional, and national incidence, prevalence, and years lived with disability for 310 diseases and injuries, 1990-2015: a systematic analysis for the global burden of disease study 2015 (vol 388, pg 1545, 2016). Lancet, 389(10064), E1-E1.

Hayward, C. (1995). Psychiatric illness and cardiovascular disease risk. Epidemiologic reviews, 17(1), 129-138.

Hazel, N., Hammen, C., Brennan, P., \& Najman, J. (2008). Early childhood adversity and adolescent depression: the mediating role of continued stress. Psychological medicine, 38(4), 581-589.

Hendricksen, M., Thomas, A. J., Ferrier, I. N., Ince, P., \& O'Brien, J. T. (2004). Neuropathological study of the dorsal raphe nuclei in late-life depression and Alzheimer's disease with and without depression. American Journal of Psychiatry, 161(6), 1096-1102.

Hildreth, C. M., Padley, J. R., Pilowsky, P. M., \& Goodchild, A. K. (2008). Impaired serotonergic regulation of heart rate may underlie reduced baroreflex sensitivity in an animal model of depression. American Journal of Physiology-Heart and Circulatory Physiology, 294(1), H474$\mathrm{H} 480$.

Hirschfeld, R. M. (2001). The comorbidity of major depression and anxiety disorders: recognition and management in primary care. Primary care companion to the Journal of clinical psychiatry, 3(6), 244.

Hollis, F., \& Kabbaj, M. (2014). Social defeat as an animal model for depression. ILAR journal, 55(2), 221-232.

Hollis, F., Wang, H., Dietz, D., Gunjan, A., \& Kabbaj, M. (2010). The effects of repeated social defeat on long-term depressive-like behavior and short-term histone modifications in the hippocampus in male Sprague-Dawley rats. Psychopharmacology, 211(1), 69-77.

Holmes, A., Yang, R. J., Lesch, K.-P., Crawley, J. N., \& Murphy, D. L. (2003). Mice lacking the serotonin transporter exhibit 5-HT $1 \mathrm{~A}$ receptor-mediated abnormalities in tests for anxiety-like behavior. Neuropsychopharmacology, 28(12), 2077. 
Homberg, J., Olivier, J., Smits, B., Mul, J., Mudde, J., Verheul, M., ... Cremers, T. (2007).

Characterization of the serotonin transporter knockout rat: a selective change in the functioning of the serotonergic system. Neuroscience, 146(4), 1662-1676.

Homberg, J. R., De Boer, S. F., Raas $\varnothing$, H. S., Olivier, J. D., Verheul, M., Ronken, E., . . Vanderschuren,

L. J. (2008). Adaptations in pre-and postsynaptic 5-HT $1 \mathrm{~A}$ receptor function and cocaine supersensitivity in serotonin transporter knockout rats. Psychopharmacology, 200(3), 367-

380.

Homberg, J. R., Schiepers, O. J., Schoffelmeer, A. N., Cuppen, E., \& Vanderschuren, L. J. (2007). Acute and constitutive increases in central serotonin levels reduce social play behaviour in periadolescent rats. Psychopharmacology, 195(2), 175.

Homberg, J. R., Schubert, D., \& Gaspar, P. (2010). New perspectives on the neurodevelopmental effects of SSRIs. Trends in pharmacological sciences, 31(2), 60-65.

Jacobs, B., Van Praag, H., \& Gage, F. (2000). Adult brain neurogenesis and psychiatry: a novel theory of depression. Molecular Psychiatry, 5(3), 262.

Jandackova, V. K., Britton, A., Malik, M., \& Steptoe, A. (2016). Heart rate variability and depressive symptoms: a cross-lagged analysis over a 10 -year period in the Whitehall II study. Psychological medicine, 46(10), 2121-2131.

Jelen, P., Soltysik, S., \& Zagrodzka, J. (2003). 22-kHz ultrasonic vocalization in rats as an index of anxiety but not fear: behavioral and pharmacological modulation of affective state. Behavioural brain research, 141(1), 63-72.

Jonnakuty, C., \& Gragnoli, C. (2008). What do we know about serotonin? Journal of cellular physiology, 217(2), 301-306.

Jordan, D. (2005). Vagal control of the heart: central serotonergic (5-HT) mechanisms. Experimental physiology, 90(2), 175-181.

Kamath, V. P., Diedrich, P., \& Hindsgaul, O. (1996). Use of diethyl squarate for the coupling of oligosaccharide amines to carrier proteins and characterization of the resulting neoglycoproteins by MALDI-TOF mass spectrometry. Glycoconjugate journal, 13(2), 315-319. Kaufman, J., \& Charney, D. (2000). Comorbidity of mood and anxiety disorders. Depression and anxiety, 12(S1), 69-76.

Kee, N., Sivalingam, S., Boonstra, R., \& Wojtowicz, J. (2002). The utility of Ki-67 and BrdU as proliferative markers of adult neurogenesis. Journal of neuroscience methods, 115(1), 97105.

Keeney, A., \& Hogg, S. (1999). Behavioural consequences of repeated social defeat in the mouse: preliminary evaluation of a potential animal model of depression. Behavioural pharmacology, 10(8), 753-764.

Keller, M. B., Hirschfeld, R., Demyttenaere, K., \& Baldwin, D. (2002). Optimizing outcomes in depression: focus on antidepressant compliance. International clinical psychopharmacology, 17(6), 265-271.

Kemp, A. H., Quintana, D. S., Felmingham, K. L., Matthews, S., \& Jelinek, H. F. (2012). Depression, comorbid anxiety disorders, and heart rate variability in physically healthy, unmedicated patients: implications for cardiovascular risk. PloS one, 7(2), e30777.

Kemp, A. H., Quintana, D. S., Gray, M. A., Felmingham, K. L., Brown, K., \& Gatt, J. M. (2010). Impact of depression and antidepressant treatment on heart rate variability: a review and metaanalysis. Biological psychiatry, 67(11), 1067-1074.

Kempermann, G., \& Kronenberg, G. (2003). Depressed new neurons?-Adult hippocampal neurogenesis and a cellular plasticity hypothesis of major depression. Biological psychiatry, 54(5), 499-503.

Kenna, G. A., Roder-Hanna, N., Leggio, L., Zywiak, W. H., Clifford, J., Edwards, S., . . Swift, R. M. (2012). Association of the 5-HTT gene-linked promoter region (5-HTTLPR) polymorphism with psychiatric disorders: review of psychopathology and pharmacotherapy. Pharmacogenomics and personalized medicine, 5, 19. 
Kepser, L.-J., \& Homberg, J. R. (2015). The neurodevelopmental effects of serotonin: a behavioural perspective. Behavioural brain research, 277, 3-13.

Kessler, R. C., Nelson, C. B., McGonagle, K. A., \& Liu, J. (1996). Comorbidity of DSM-III-R major depressive disorder in the general population: Results from the US National Comorbidity

Survey. The British journal of psychiatry.

Kheirbek, M. A., Klemenhagen, K. C., Sahay, A., \& Hen, R. (2012). Neurogenesis and generalization: a new approach to stratify and treat anxiety disorders. Nature neuroscience, 15(12), 1613.

Kidwell, M., \& Ellenbroek, B. A. (2018a). Heart and soul: heart rate variability and major depression. Behavioural pharmacology, 29(2), 152-164.

Kidwell, M., \& Ellenbroek, B. A. (2018b). Heart and soul: heart rate variability and major depression. Behavioural pharmacology, 29(2 and 3-Special Issue), 152-164.

Kirsch, I., Deacon, B. J., Huedo-Medina, T. B., Scoboria, A., Moore, T. J., \& Johnson, B. T. (2008). Initial severity and antidepressant benefits: a meta-analysis of data submitted to the Food and Drug Administration. PLoS medicine, 5(2), e45.

Kiser, D., SteemerS, B., Branchi, I., \& Homberg, J. R. (2012). The reciprocal interaction between serotonin and social behaviour. Neuroscience \& Biobehavioral Reviews, 36(2), 786-798.

Kishimoto, K., Koyama, S., \& Akaike, N. (2001). Synergistic $\mu$-opioid and 5-HT1A presynaptic inhibition of GABA release in rat periaqueductal gray neurons. Neuropharmacology, 41(5), 529-538.

Kola, I., \& Landis, J. (2004). Can the pharmaceutical industry reduce attrition rates? Nature reviews Drug discovery, 3(8), 711.

Krishnan, V., Han, M.-H., Graham, D. L., Berton, O., Renthal, W., Russo, S. J., . . Lagace, D. C. (2007). Molecular adaptations underlying susceptibility and resistance to social defeat in brain reward regions. Cell, 131(2), 391-404.

Krishnan, V., \& Nestler, E. J. (2008). The molecular neurobiology of depression. Nature, 455(7215), 894.

Kruesi, M. J., Rapoport, J. L., Hamburger, S., Hibbs, E., Potter, W. Z., Lenane, M., \& Brown, G. L. (1990). Cerebrospinal fluid monoamine metabolites, aggression, and impulsivity in disruptive behavior disorders of children and adolescents. Archives of general psychiatry, 47(5), 419426.

Kuhn, H. G., Dickinson-Anson, H., \& Gage, F. H. (1996). Neurogenesis in the dentate gyrus of the adult rat: age-related decrease of neuronal progenitor proliferation. Journal of Neuroscience, 16(6), 2027-2033.

Kupfer, D. J., Frank, E., \& Phillips, M. L. (2012). Major depressive disorder: new clinical, neurobiological, and treatment perspectives. The Lancet, 379(9820), 1045-1055.

Lam, T. P., Goldberg, D., Dowell, A. C., Fortes, S., Mbatia, J., Minhas, F. A., \& Klinkman, M. (2012). Proposed new diagnoses of anxious depression and bodily stress syndrome in ICD-11-PHC: an international focus group study. Family practice, 30(1), 76-87.

Landgraf, R., \& Wigger, A. (2002). High vs low anxiety-related behavior rats: an animal model of extremes in trait anxiety. Behavior genetics, 32(5), 301-314.

Lauder, J. M. (1990). Ontogeny of the Serotonergic System in the Rat: Serotonin as a Developmental Signal a. Annals of the New York Academy of Sciences, 600(1), 297-313.

Lebrand, C., Cases, O., Adelbrecht, C., Doye, A., Alvarez, C., El Mestikawy, S., . . Gaspar, P. (1996). Transient uptake and storage of serotonin in developing thalamic neurons. Neuron, 17(5), 823-835.

Lesch, K.-P., Bengel, D., Heils, A., Sabol, S. Z., Greenberg, B. D., Petri, S., . . Murphy, D. L. (1996). Association of anxiety-related traits with a polymorphism in the serotonin transporter gene regulatory region. Science, 274(5292), 1527-1531.

Lewinsohn, P. M., Mischel, W., Chaplin, W., \& Barton, R. (1980). Social competence and depression: The role of illusory self-perceptions. Journal of abnormal psychology, 89(2), 203. 
Licht, C. M., De Geus, E. J., Van Dyck, R., \& Penninx, B. W. (2009). Association between anxiety disorders and heart rate variability in The Netherlands Study of Depression and Anxiety

(NESDA). Psychosomatic medicine, 71(5), 508-518.

Lima, S. L., \& Dill, L. M. (1990). Behavioral decisions made under the risk of predation: a review and prospectus. Canadian journal of zoology, 68(4), 619-640.

Lira, A., Zhou, M., Castanon, N., Ansorge, M. S., Gordon, J. A., Francis, J. H., ... Arango, V. (2003). Altered depression-related behaviors and functional changes in the dorsal raphe nucleus of serotonin transporter-deficient mice. Biological psychiatry, 54(10), 960-971.

Lister, R. G. (1987). The use of a plus-maze to measure anxiety in the mouse. Psychopharmacology, 92(2), 180-185.

Lizarraga, L. E., Phan, A. V., Cholanians, A. B., Herndon, J. M., Lau, S. S., \& Monks, T. J. (2014). Serotonin reuptake transporter deficiency modulates the acute thermoregulatory and locomotor activity response to 3, 4-( \pm )-methylenedioxymethamphetamine, and attenuates depletions in serotonin levels in SERT-KO rats. Toxicological Sciences, 139(2), 421-431.

Locher, C., Koechlin, H., Zion, S. R., Werner, C., Pine, D. S., Kirsch, I., ... Kossowsky, J. (2017). Efficacy and safety of selective serotonin reuptake inhibitors, serotonin-norepinephrine reuptake inhibitors, and placebo for common psychiatric disorders among children and adolescents: a systematic review and meta-analysis. JAMA psychiatry, 74(10), 1011-1020.

Logothetis, N. K. (2008). What we can do and what we cannot do with fMRI. Nature, 453(7197), 869. Lokhandwala, M. F., \& Barrett, R. J. (1983). Dopamine receptor agonists in cardiovascular therapy. Drug development research, 3(4), 299-310.

Longland, W. S. (1991). Risk of predation and food consumption by black-tailed jackrabbits. Journal of Range Management, 447-450.

Longo, L. P., \& Johnson, B. (2000). Addiction: Part I. Benzodiazepines-side effects, abuse risk and alternatives. American family physician, 61(7), 2121-2128.

Lopez-Leon, S., Janssens, A., Ladd, A. G.-Z., Del-Favero, J., Claes, S., Oostra, B., \& Van Duijn, C. (2008). Meta-analyses of genetic studies on major depressive disorder. Molecular Psychiatry, 13(8), 772.

Maesen, B., Nijs, J., Maessen, J., Allessie, M., \& Schotten, U. (2011). Post-operative atrial fibrillation: a maze of mechanisms. Europace, 14(2), 159-174.

Marangell, L. B., Rush, A. J., George, M. S., Sackeim, H. A., Johnson, C. R., Husain, M. M., . . Lisanby, S. H. (2002). Vagus nerve stimulation (VNS) for major depressive episodes: one year outcomes. Biological psychiatry, 51(4), 280-287.

Martinowich, K., \& Lu, B. (2008). Interaction between BDNF and serotonin: role in mood disorders. Neuropsychopharmacology, 33(1), 73.

Maslow, A. H. (1943). A theory of human motivation. Psychological review, $50(4), 370$.

Matsuda, N., Jesmin, S., Takahashi, Y., Hatta, E., Kobayashi, M., Matsuyama, K., . . Fukui, H. (2004). Histamine $\mathrm{H} 1$ and $\mathrm{H} 2$ receptor gene and protein levels are differentially expressed in the hearts of rodents and humans. Journal of Pharmacology and Experimental Therapeutics, 309(2), 786-795.

Mazzanti, C. M., Lappalainen, J., Long, J. C., Bengel, D., Naukkarinen, H., Eggert, M., . . . Goldman, D. (1998). Role of the serotonin transporter promoter polymorphism in anxiety-related traits. Archives of general psychiatry, 55(10), 936-940.

Medzihradszky, K. F., Campbell, J. M., Baldwin, M. A., Falick, A. M., Juhasz, P., Vestal, M. L., \& Burlingame, A. L. (2000). The characteristics of peptide collision-induced dissociation using a high-performance MALDI-TOF/TOF tandem mass spectrometer. Analytical Chemistry, 72(3), 552-558.

Melartin, T. K., Rytsälä, H. J., Leskelä, U. S., Lestelä-Mielonen, P. S., Sokero, T. P., \& Isometsä, E. T. (2002). Current comorbidity of psychiatric disorders among DSM-IV major depressive disorder patients in psychiatric care in the Vantaa Depression Study. The Journal of clinical psychiatry. 
Ming, G.-I., \& Song, H. (2005). Adult neurogenesis in the mammalian central nervous system. Annu. Rev. Neurosci., 28, 223-250.

Moffitt, T. E., Harrington, H., Caspi, A., Kim-Cohen, J., Goldberg, D., Gregory, A. M., \& Poulton, R. (2007). Depression and generalized anxiety disorder: cumulative and sequential comorbidity in a birth cohort followed prospectively to age 32 years. Archives of general psychiatry, 64(6), 651-660.

Mohr, D. C., Goodkin, D. E., Gatto, N., \& Van Der Wende, J. (1997). Depression, coping and level of neurological impairment in multiple sclerosis. Multiple Sclerosis Journal, 3(4), 254-258.

Molina, E., Cervilla, J., Rivera, M., Torres, F., Bellón, J. Á., Moreno, B., . . Gutierrez, B. (2011). Polymorphic variation at the serotonin 1-A receptor gene is associated with comorbid depression and generalized anxiety. Psychiatric Genetics, 21(4), 195-201.

Montañez, S., Munn, J. L., Owens, W. A., Horton, R. E., \& Daws, L. C. (2014). 5-HT1B receptor modulation of the serotonin transporter in vivo: studies using KO mice. Neurochemistry international, 73, 127-131.

Moy, S. S., Nadler, J. J., Young, N. B., Nonneman, R. J., Grossman, A. W., Murphy, D. L., . . Lauder, J. M. (2009). Social approach in genetically engineered mouse lines relevant to autism. Genes, Brain and Behavior, 8(2), 129-142.

Munafò, M. R., Brown, S. M., \& Hariri, A. R. (2008). Serotonin transporter (5-HTTLPR) genotype and amygdala activation: a meta-analysis. Biological psychiatry, 63(9), 852-857.

Murakami, F., Shimomura, T., Kotani, K., Ikawa, S., Nanba, E., \& Adachi, K. (1999). Anxiety traits associated with a polymorphism in the serotonin transporter gene regulatory region in the Japanese. Journal of human genetics, 44(1), 15.

Murakami, H., Matsunaga, M., \& Ohira, H. (2009). Association of serotonin transporter gene polymorphism and emotion regulation. Neuroreport, 20(4), 414-418.

Murphy, D. L., \& Lesch, K.-P. (2008). Targeting the murine serotonin transporter: insights into human neurobiology. Nature Reviews Neuroscience, 9(2), 85.

Musselman, D. L., Evans, D. L., \& Nemeroff, C. B. (1998). The relationship of depression to cardiovascular disease: epidemiology, biology, and treatment. Archives of general psychiatry, 55(7), 580-592.

Nahas, Z., Marangell, L. B., Husain, M. M., Rush, A. J., Sackeim, H. A., Lisanby, S. H., . . G George, M. S. (2005). Two-year outcome of vagus nerve stimulation (VNS) for treatment of major depressive episodes. The Journal of clinical psychiatry, 66(9), 1097-1104.

Nakamura, M., Ueno, S., Sano, A., \& Tanabe, H. (2000). The human serotonin transporter gene linked polymorphism (5-HTTLPR) shows ten novel allelic variants. Molecular Psychiatry, 5(1), 32.

Narita, N., Kato, M., Tazoe, M., Miyazaki, K., Narita, M., \& Okado, N. (2002). Increased monoamine concentration in the brain and blood of fetal thalidomide-and valproic acid-exposed rat: putative animal models for autism. Pediatric research, 52(4), 576.

Nemeroff, C. B., Mayberg, H. S., Krahl, S. E., McNamara, J., Frazer, A., Henry, T. R., . . Brannan, S. K. (2006). VNS therapy in treatment-resistant depression: clinical evidence and putative neurobiological mechanisms. Neuropsychopharmacology, 31(7), 1345.

Neumann, I., Wegener, G., Homberg, J., Cohen, H., Slattery, D., Zohar, J., . . Mathé, A. (2011). Animal models of depression and anxiety: What do they tell us about human condition?

Progress in Neuro-Psychopharmacology and Biological Psychiatry, 35(6), 1357-1375.

New, A. S., Buchsbaum, M. S., Hazlett, E. A., Goodman, M., Koenigsberg, H. W., Lo, J., . . O'Flynn, K. (2004). Fluoxetine increases relative metabolic rate in prefrontal cortex in impulsive aggression. Psychopharmacology, 176(3-4), 451-458.

Nezlek, J. B., Hampton, C. P., \& Shean, G. D. (2000). Clinical depression and day-to-day social interaction in a community sample. Journal of abnormal psychology, 109(1), 11.

Nezlek, J. B., Imbrie, M., \& Shean, G. D. (1994). Depression and everyday social interaction. Journal of Personality and Social Psychology, 67(6), 1101. 
Nicholson, A., Kuper, H., \& Hemingway, H. (2006). Depression as an aetiologic and prognostic factor in coronary heart disease: a meta-analysis of 6362 events among 146538 participants in 54 observational studies. European heart journal, 27(23), 2763-2774.

Nutt, D. J., Forshall, S., Bell, C., Rich, A., Sandford, J., Nash, J., \& Argyropoulos, S. (1999). Mechanisms of action of selective serotonin reuptake inhibitors in the treatment of psychiatric disorders. European Neuropsychopharmacology, 9, S81-S86.

O'reardon, J. P., Brunswick, D. J., \& Amsterdam, J. D. (2000). Treatment-resistant depression in the age of serotonin: Evolving strategies. Current Opinion in psychiatry, 13(1), 93-98.

O'Leary, O. F., Ogbonnaya, E. S., Felice, D., Levone, B. R., Conroy, L. C., Fitzgerald, P., . . Dinan, T. G. (2018). The vagus nerve modulates BDNF expression and neurogenesis in the hippocampus. European Neuropsychopharmacology, 28(2), 307-316.

Oakly, A., Brox, B., Schenk, S., \& Ellenbroek, B. (2014). A genetic deletion of the serotonin transporter greatly enhances the reinforcing properties of MDMA in rats. Molecular Psychiatry, 19(5), 534.

Oberlander, T., Gingrich, J., \& Ansorge, M. (2009). Sustained neurobehavioral effects of exposure to SSRI antidepressants during development: molecular to clinical evidence. Clinical Pharmacology \& Therapeutics, 86(6), 672-677.

Oehen, P., Traber, R., Widmer, V., \& Schnyder, U. (2013). A randomized, controlled pilot study of MDMA ( \pm 3 , 4-Methylenedioxymethamphetamine)-assisted psychotherapy for treatment of resistant, chronic Post-Traumatic Stress Disorder (PTSD). Journal of psychopharmacology, 27(1), 40-52.

Olivier, J., Van Der Hart, M., Van Swelm, R., Dederen, P., Homberg, J., Cremers, T., . . Ellenbroek, B. (2008). A study in male and female 5-HT transporter knockout rats: an animal model for anxiety and depression disorders. Neuroscience, 152(3), 573-584.

Olivier, J. D., Cools, A. R., Olivier, B., Homberg, J. R., Cuppen, E., \& Ellenbroek, B. A. (2008). Stressinduced hyperthermia and basal body temperature are mediated by different 5-HT1A receptor populations: A study in SERT knockout rats. European journal of pharmacology, 590(1-3), 190-197.

Organization, W. H. (2017). Depression and other common mental disorders: global health estimates.

Ottaviani, C., Watson, D. R., Meeten, F., Makovac, E., Garfinkel, S. N., \& Critchley, H. D. (2016). Neurobiological substrates of cognitive rigidity and autonomic inflexibility in generalized anxiety disorder. Biological psychology, 119, 31-41.

Overstreet, D. H., \& Wegener, G. (2013). The flinders sensitive line rat model of depression -25 years and still producing. Pharmacological reviews, 65(1), 143-155.

Panel, D. G. (1993). Depression in Primary Care: volume 2 treatment of major depression. Clinical practice guideline.

Paxinos, G., \& Watson, C. (1998). The rat brain atlas in stereotaxic coordinates. San Diego: Academic.

Penninx, B. W. (2017). Depression and cardiovascular disease: epidemiological evidence on their linking mechanisms. Neuroscience \& Biobehavioral Reviews, 74, 277-286.

Perazella, M. A. (2000). Drug-induced hyperkalemia: old culprits and new offenders. The American journal of medicine, 109(4), 307-314.

Persico, A., Altamura, C., Calia, E., Puglisi-Allegra, S., Ventura, R., Lucchese, F., \& Keller, F. (2000). Serotonin depletion and barrel cortex development: impact of growth impairment vs. serotonin effects on thalamocortical endings. Cerebral cortex, 10(2), 181-191.

Petersen, C. C. (2007). The functional organization of the barrel cortex. Neuron, 56(2), 339-355.

Pigott, H. E., Leventhal, A. M., Alter, G. S., \& Boren, J. J. (2010). Efficacy and effectiveness of antidepressants: current status of research. Psychotherapy and psychosomatics, 79(5), 267279.

Polter, A. M., \& Li, X. (2010). 5-HT1A receptor-regulated signal transduction pathways in brain. Cellular signalling, 22(10), 1406-1412. 
Porsolt, R., Le Pichon, M., \& Jalfre, M. (1977). Depression: a new animal model sensitive to antidepressant treatments. Nature, 266(5604), 730.

Pothion, S., Bizot, J.-C., Trovero, F., \& Belzung, C. (2004). Strain differences in sucrose preference and in the consequences of unpredictable chronic mild stress. Behavioural brain research, 155(1), 135-146.

Poulton, R., Moffitt, T. E., \& Silva, P. A. (2015). The Dunedin Multidisciplinary Health and Development Study: overview of the first 40 years, with an eye to the future. Social Psychiatry and Psychiatric Epidemiology, 50(5), 679-693.

Pratt, L. A., Ford, D. E., Crum, R. M., Armenian, H. K., Gallo, J. J., \& Eaton, W. W. (1996). Depression, psychotropic medication, and risk of myocardial infarction: prospective data from the Baltimore ECA follow-up. circulation, 94(12), 3123-3129.

Pyszczynski, T., Hamilton, J. C., Herring, F. H., \& Greenberg, J. (1989). Depression, self-focused attention, and the negative memory bias. Journal of Personality and Social Psychology, 57(2), 351.

Rajkowska, G. (2000). Postmortem studies in mood disorders indicate altered numbers of neurons and glial cells. Biological psychiatry, 48(8), 766-777.

Ramboz, S., Oosting, R., Amara, D. A., Kung, H. F., Blier, P., Mendelsohn, M., . . Hen, R. (1998). Serotonin receptor $1 \mathrm{~A}$ knockout: an animal model of anxiety-related disorder. Proceedings of the National Academy of Sciences, 95(24), 14476-14481.

Ramos, A. (2008). Animal models of anxiety: do I need multiple tests? Trends in pharmacological sciences, 29(10), 493-498.

Reif, A., Schmitt, A., Fritzen, S., \& Lesch, K.-P. (2007). Neurogenesis and schizophrenia: dividing neurons in a divided mind? European archives of psychiatry and clinical neuroscience, 257(5), 290-299.

Revest, J., Dupret, D., Koehl, M., Funk-Reiter, C., Grosjean, N., Piazza, P., \& Abrous, D. (2009). Adult hippocampal neurogenesis is involved in anxiety-related behaviors. Molecular Psychiatry, 14(10), 959.

Rosenthal, N. E., Mazzanti, C. M., Barnett, R. L., Hardin, T. A., Turner, E. H., Lam, G. K., . . Goldman, D. (1998). Role of serotonin transporter promoter repeat length polymorphism (5-HTTLPR) in seasonality and seasonal affective disorder. Molecular Psychiatry, 3(2), 175.

Roy-Byrne, P., Wingerson, D., Cowley, D., \& Dager, S. (1993). Psychopharmacologic treatment of panic, generalized anxiety disorder, and social phobia. Psychiatric Clinics of North America.

Ruiz Vargas, E., Sörös, P., Shoemaker, J. K., \& Hachinski, V. (2016). Human cerebral circuitry related to cardiac control: a neuroimaging meta-analysis. Annals of Neurology, 79(5), 709-716.

Rygula, R., Abumaria, N., Flügge, G., Fuchs, E., Rüther, E., \& Havemann-Reinecke, U. (2005). Anhedonia and motivational deficits in rats: impact of chronic social stress. Behavioural brain research, 162(1), 127-134.

Sackeim, H. A., Rush, A. J., George, M. S., Marangell, L. B., Husain, M. M., Nahas, Z., . . Haines, S. (2001). Vagus nerve stimulation (VNS'TM) for treatment-resistant depression: efficacy, side effects, and predictors of outcome. Neuropsychopharmacology, 25(5), 713-728.

Sahay, A., \& Hen, R. (2007). Adult hippocampal neurogenesis in depression. Nature neuroscience, 10(9), 1110.

Sanders, A. C., Hussain, A. J., Hen, R., \& Zhuang, X. (2007). Chronic blockade or constitutive deletion of the serotonin transporter reduces operant responding for food reward. Neuropsychopharmacology, 32(11), 2321.

Santos, J. R. A. (1999). Cronbach's alpha: A tool for assessing the reliability of scales. Journal of extension, 37(2), 1-5.

Savitz, J. B., \& Ramesar, R. S. (2004). Genetic variants implicated in personality: a review of the more promising candidates. American Journal of Medical Genetics Part B: Neuropsychiatric Genetics, 131(1), 20-32. 
Schneider, P., Ho, Y.-J., Spanagel, R., \& Pawlak, C. R. (2011). A novel elevated plus-maze procedure to avoid the one-trial tolerance problem. Frontiers in behavioral neuroscience, 5, 43.

Schubert, D., Martens, G., \& Kolk, S. (2015). Molecular underpinnings of prefrontal cortex development in rodents provide insights into the etiology of neurodevelopmental disorders.

Molecular Psychiatry, 20(7), 795.

Schwarting, R., \& Pawlak, C. (2004). Behavioral neuroscience in the rat: taking the individual into account. Meth Find Exp Clin Pharmacol, 26(S2), 17-22.

Serra, M., Pisu, M., Floris, I., \& Biggio, G. (2005). Social isolation-induced changes in the hypothalamic-pituitary-adrenal axis in the rat. Stress, 8(4), 259-264.

Serretti, A., Lilli, R., Lorenzi, C., Lattuada, E., Cusin, C., \& Smeraldi, E. (2002). Serotonin transporter gene (5-HTTLPR) and major psychoses. Molecular Psychiatry, 7(1), 95.

Sessa, B. (2007). Is there a case for MDMA-assisted psychotherapy in the UK? Journal of psychopharmacology, 21(2), 220-224.

Setiawan, E., Attwells, S., Wilson, A. A., Mizrahi, R., Rusjan, P. M., Miler, L., . . Houle, S. (2018). Association of translocator protein total distribution volume with duration of untreated major depressive disorder: a cross-sectional study. The Lancet Psychiatry, 5(4), 339-347.

Sevcencu, C., \& Struijk, J. J. (2010). Autonomic alterations and cardiac changes in epilepsy. Epilepsia, 51(5), 725-737.

Sgoifo, A., Stilli, D., Medici, D., Gallo, P., Aimi, B., \& Musso, E. (1996). Electrode positioning for reliable telemetry ECG recordings during social stress in unrestrained rats. Physiology \& behavior, 60(6), 1397-1401.

Shariatgorji, M., Nilsson, A., Goodwin, R. J., Källback, P., Schintu, N., Zhang, X., . . Andren, P. E. (2014). Direct targeted quantitative molecular imaging of neurotransmitters in brain tissue sections. Neuron, 84(4), 697-707.

Shariatgorji, M., Nilsson, A., Källback, P., Karlsson, O., Zhang, X., Svenningsson, P., \& Andren, P. E. (2015). Pyrylium salts as reactive matrices for MALDI-MS imaging of biologically active primary amines. Journal of the American Society for Mass Spectrometry, 26(6), 934-939.

Smeraldi, E., Zanardi, R., Benedetti, F., Di Bella, D., Perez, J., \& Catalano, M. (1998). Polymorphism within the promoter of the serotonin transporter gene and antidepressant efficacy of

fluvoxamine. Molecular Psychiatry, 3(6), 508.

Smith, C., \& Reichman, O. (1984). The evolution of food caching by birds and mammals. Annual review of ecology and systematics, 15(1), 329-351.

Smits, B. M., Mudde, J. B., van de Belt, J., Verheul, M., Olivier, J., Homberg, J., . . Plasterk, R. H. (2006). Generation of gene knockouts and mutant models in the laboratory rat by ENUdriven target-selected mutagenesis. Pharmacogenetics and genomics, 16(3), 159-169.

Smoller, J. W. (2016). The genetics of stress-related disorders: PTSD, depression, and anxiety disorders. Neuropsychopharmacology, 41(1), 297.

Sorrells, S. F., Paredes, M. F., Cebrian-Silla, A., Sandoval, K., Qi, D., Kelley, K. W., . . Auguste, K. I.

(2018). Human hippocampal neurogenesis drops sharply in children to undetectable levels in adults. Nature, 555(7696), 377.

Souery, D., Papakostas, G. I., \& Trivedi, M. H. (2006). Treatment-resistant depression. The Journal of clinical psychiatry, 67, 16-22.

Stanton, W. R. (1996). From child to adult: The Dunedin multidisciplinary health and development study: Oxford University Press, USA.

Steenbergen, L., Jongkees, B. J., Sellaro, R., \& Colzato, L. S. (2016). Tryptophan supplementation modulates social behavior: a review. Neuroscience \& Biobehavioral Reviews, 64, 346-358.

Stice, E., Ragan, J., \& Randall, P. (2004). Prospective relations between social support and depression: Differential direction of effects for parent and peer support? Journal of abnormal psychology, 113(1), 155. 
Stoltenberg, S. F., Christ, C. C., \& Carlo, G. (2013). Afraid to help: social anxiety partially mediates the association between 5-HTTLPR triallelic genotype and prosocial behavior. Social neuroscience, 8(5), 400-406.

Stravynski, A., \& Boyer, R. (2001). Loneliness in relation to suicide ideation and parasuicide: a population-wide study. Suicide and Life-Threatening Behavior, 31(1), 32-40.

Taupin, P. (2007). BrdU immunohistochemistry for studying adult neurogenesis: paradigms, pitfalls, limitations, and validation. Brain research reviews, 53(1), 198-214.

Tauscher, J., Bagby, R. M., Javanmard, M., Christensen, B. K., Kasper, S., \& Kapur, S. (2001). Inverse relationship between serotonin 5-HT1A receptor binding and anxiety: a [11C] WAY-100635 PET investigation in healthy volunteers. American Journal of Psychiatry, 158(8), 1326-1328.

Taylor, S. E., Way, B. M., Welch, W. T., Hilmert, C. J., Lehman, B. J., \& Eisenberger, N. I. (2006). Early family environment, current adversity, the serotonin transporter promoter polymorphism, and depressive symptomatology. Biological psychiatry, 60(7), 671-676.

Teo, A. R., Choi, H., \& Valenstein, M. (2013). Social relationships and depression: ten-year follow-up from a nationally representative study. PloS one, 8(4), e62396.

Thayer, J. F., Yamamoto, S. S., \& Brosschot, J. F. (2010). The relationship of autonomic imbalance, heart rate variability and cardiovascular disease risk factors. International journal of cardiology, 141(2), 122-131.

Thiele, A., \& Rehman, H. U. (2008). Hyperkalemia caused by penicillin. The American journal of medicine, 121(8), e1-e2.

Thompson, B., Leonard, K. C., \& Brudzynski, S. M. (2006). Amphetamine-induced $50 \mathrm{kHz}$ calls from rat nucleus accumbens: a quantitative mapping study and acoustic analysis. Behavioural brain research, 168(1), 64-73.

Tornatzky, W., \& Miczek, K. A. (1993). Long-term impairment of autonomic circadian rhythms after brief intermittent social stress. Physiology \& behavior, 53(5), 983-993.

Trezza, V., Damsteegt, R., \& Vanderschuren, L. J. (2009). Conditioned place preference induced by social play behavior: parametrics, extinction, reinstatement and disruption by methylphenidate. European Neuropsychopharmacology, 19(9), 659-669.

Tzschentke, T. M. (1998). Measuring reward with the conditioned place preference paradigm: a comprehensive review of drug effects, recent progress and new issues. Progress in neurobiology, 56(6), 613-672.

Tzschentke, T. M. (2007). Review on CPP: Measuring reward with the conditioned place preference (CPP) paradigm: update of the last decade. Addiction biology, 12(3-4), 227-462.

Uher, R., Mors, O., Rietschel, M., Rajewska-Rager, A., Petrovic, A., Zobel, A., . . Farmer, A. (2011). Early and delayed onset of response to antidepressants in individual trajectories of change during treatment of major depression: a secondary analysis of data from the Genome-Based Therapeutic Drugs for Depression (GENDEP) study. The Journal of clinical psychiatry.

Ulrich-Lai, Y. M., Christiansen, A. M., Ostrander, M. M., Jones, A. A., Jones, K. R., Choi, D. C., . . Davis, J. F. (2010). Pleasurable behaviors reduce stress via brain reward pathways. Proceedings of the National Academy of Sciences, 201007740.

van der Kooy, K. G., van Hout, H. P., van Marwijk, H. W., de Haan, M., Stehouwer, C. D., \& Beekman, A. T. (2006). Differences in heart rate variability between depressed and non-depressed elderly. International journal of geriatric psychiatry, 21(2), 147-150.

Vanderschuren, L. J., Niesink, R. J., Spruijt, B. M., \& Van Ree, J. M. (1995). Influence of environmental factors on social play behavior of juvenile rats. Physiology \& behavior, 58(1), 119-123.

Verdolin, J. L. (2006). Meta-analysis of foraging and predation risk trade-offs in terrestrial systems. Behavioral Ecology and Sociobiology, 60(4), 457-464.

Vitalis, T., \& Parnavelas, J. G. (2003). The role of serotonin in early cortical development. Developmental neuroscience, 25(2-4), 245-256. 
Vollenweider, F. X., Liechti, M. E., Gamma, A., Greer, G., \& Geyer, M. (2002). Acute psychological and neurophysiological effects of MDMA in humans. Journal of psychoactive drugs, 34(2), 171-

184.

Wall, P., \& Messier, C. (2001). Methodological and conceptual issues in the use of the elevated plusmaze as a psychological measurement instrument of animal anxiety-like behavior. Neuroscience \& Biobehavioral Reviews, 25(3), 275-286.

Wallace, J. A., \& Lauder, J. M. (1983). Development of the serotonergic system in the rat embryo: an immunocytochemical study. Brain research bulletin, 10(4), 459-479.

Wankerl, M., Miller, R., Kirschbaum, C., Hennig, J., Stalder, T., \& Alexander, N. (2014). Effects of genetic and early environmental risk factors for depression on serotonin transporter expression and methylation profiles. Translational psychiatry, 4(6), e402.

Wassertheil-Smoller, S., Applegate, W. B., Berge, K., Chang, C. J., Davis, B. R., Grimm, R., ... Schron, E. (1996). Change in depression as a precursor of cardiovascular events. Archives of internal medicine, 156(5), 553-561.

Wegiel, J., Kuchna, I., Nowicki, K., Imaki, H., Wegiel, J., Marchi, E., . . Bobrowicz, T. W. (2010). The neuropathology of autism: defects of neurogenesis and neuronal migration, and dysplastic changes. Acta neuropathologica, 119(6), 755-770.

Weiss, I. C., Pryce, C. R., Jongen-Rêlo, A. L., Nanz-Bahr, N. I., \& Feldon, J. (2004). Effect of social isolation on stress-related behavioural and neuroendocrine state in the rat. Behavioural brain research, 152(2), 279-295.

Willner, P. (2005). Chronic mild stress (CMS) revisited: consistency and behavioural-neurobiological concordance in the effects of CMS. Neuropsychobiology, 52(2), 90-110.

Willner, P., Towell, A., Sampson, D., Sophokleous, S., \& Muscat, R. (1987). Reduction of sucrose preference by chronic unpredictable mild stress, and its restoration by a tricyclic antidepressant. Psychopharmacology, 93(3), 358-364.

Witteveen, J. S., Middelman, A., van Hulten, J. A., Martens, G. J. M., Homberg, J. R., \& Kolk, S. M. (2013). Lack of serotonin reuptake during brain development alters rostral raphe-prefrontal network formation. Frontiers in cellular neuroscience, 7, 143.

Wood, P. L. (2014). Mass spectrometry strategies for clinical metabolomics and lipidomics in psychiatry, neurology, and neuro-oncology. Neuropsychopharmacology, 39(1), 24.

Woods, S. C., \& Begg, D. P. (2015). Regulation of the motivation to eat. In Behavioral Neuroscience of Motivation (pp. 15-34): Springer.

Yamakawa, K., Matsunaga, M., Isowa, T., \& Ohira, H. (2015). Serotonin transporter gene polymorphism modulates inflammatory cytokine responses during acute stress. Scientific reports, 5, 13852.

Yasuma, F., \& Hayano, J.-i. (2004). Respiratory sinus arrhythmia: why does the heartbeat synchronize with respiratory rhythm? Chest, 125(2), 683-690.

Yeh, M.-L., Chung, Y.-C., Hsu, L.-C., \& Hung, S.-H. (2017). Effect of transcutaneous acupoint electrical stimulation on post-hemorrhoidectomy-associated pain, anxiety, and heart rate variability: a randomized-controlled study. Clinical nursing research, 1054773816685745.

Young, H. A., Cousins, A. L., Watkins, H. T., \& Benton, D. (2017). Is the link between depressed mood and heart rate variability explained by disinhibited eating and diet? Biological psychology, 123, 94-102.

Young, S. N., \& Leyton, M. (2002). The role of serotonin in human mood and social interaction: insight from altered tryptophan levels. Pharmacology Biochemistry and Behavior, 71(4), 857865.

Zhang, J. (2007). Effect of age and sex on heart rate variability in healthy subjects. Journal of manipulative and physiological therapeutics, 30(5), 374-379. 
Appendices

Appendix 1:

Means and Standard Deviations for Chasing

\begin{tabular}{|c|c|c|c|c|c|c|c|}
\hline & & Day 1 & Day 2 & Day 3 & Day 4 & Day 5 & Overall \\
\hline \multirow{2}{*}{ SERT +/+ } & Mean & $29.62 \%$ & $25.07 \%$ & $24.96 \%$ & $22.85 \%$ & $21.36 \%$ & $24.77 \%$ \\
\hline & Standard Deviation & $3.90 \%$ & $0.62 \%$ & $2.52 \%$ & $1.26 \%$ & $2.95 \%$ & $1.39 \%$ \\
\hline \multirow{2}{*}{ SERT +/- } & Mean & $26.15 \%$ & $22.45 \%$ & $26.35 \%$ & $19.20 \%$ & $22.47 \%$ & $23.32 \%$ \\
\hline & Standard Deviation & $2.59 \%$ & $0.13 \%$ & $1.32 \%$ & $1.54 \%$ & $1.24 \%$ & $1.33 \%$ \\
\hline \multirow{2}{*}{ SERT -/- } & Mean & $19.33 \%$ & $17.39 \%$ & $21.31 \%$ & $23.44 \%$ & $26.56 \%$ & $21.61 \%$ \\
\hline & Standard Deviation & $2.72 \%$ & $2.57 \%$ & $2.31 \%$ & $3.38 \%$ & $2.64 \%$ & $1.60 \%$ \\
\hline
\end{tabular}

Appendix 2:

Means and Standard Deviations for Pinning

\begin{tabular}{|c|c|c|c|c|c|c|c|}
\hline & & Day 1 & Day 2 & Day 3 & Day 4 & Day 5 & Overall \\
\hline \multirow{2}{*}{ SERT +/+ } & Mean & $0.00 \%$ & $1.40 \%$ & $1.89 \%$ & $2.60 \%$ & $4.16 \%$ & $2.01 \%$ \\
\hline & Standard Deviation & $0.00 \%$ & $0.19 \%$ & $0.75 \%$ & $0.95 \%$ & $2.28 \%$ & $0.68 \%$ \\
\hline \multirow{2}{*}{ SERT +/- } & Mean & $0.16 \%$ & $1.16 \%$ & $2.91 \%$ & $2.02 \%$ & $2.40 \%$ & $1.73 \%$ \\
\hline & Standard Deviation & $0.10 \%$ & $0.72 \%$ & $0.94 \%$ & $0.91 \%$ & $0.15 \%$ & $0.48 \%$ \\
\hline \multirow{2}{*}{ SERT -/- } & Mean & $0.00 \%$ & $0.21 \%$ & $0.43 \%$ & $0.73 \%$ & $2.59 \%$ & $0.79 \%$ \\
\hline & Standard Deviation & $0.00 \%$ & $0.14 \%$ & $0.21 \%$ & $0.40 \%$ & $1.64 \%$ & $0.47 \%$ \\
\hline
\end{tabular}

Appendix 3:

Means and Standard Deviations for Pouncing

\begin{tabular}{|c|c|c|c|c|c|c|c|}
\hline & & Day 1 & Day 2 & Day 3 & Day 4 & Day 5 & Overall \\
\hline \multirow{2}{*}{ SERT +/+ } & Mean & $25.08 \%$ & $34.97 \%$ & $38.97 \%$ & $44.00 \%$ & $44.19 \%$ & $37.44 \%$ \\
\hline & Standard Deviation & $2.78 \%$ & $4.28 \%$ & $7.59 \%$ & $1.69 \%$ & $6.25 \%$ & $3.53 \%$ \\
\hline \multirow{2}{*}{ SERT +/- } & Mean & $28.25 \%$ & $34.92 \%$ & $42.10 \%$ & $40.50 \%$ & $28.51 \%$ & $34.86 \%$ \\
\hline & Standard Deviation & $0.40 \%$ & $1.23 \%$ & $1.43 \%$ & $0.65 \%$ & $3.56 \%$ & $2.90 \%$ \\
\hline \multirow{2}{*}{ SERT -/- } & Mean & $28.91 \%$ & $37.98 \%$ & $42.73 \%$ & $38.92 \%$ & $38.22 \%$ & $37.35 \%$ \\
\hline & Standard Deviation & $2.29 \%$ & $1.40 \%$ & $2.83 \%$ & $2.28 \%$ & $3.11 \%$ & $2.28 \%$ \\
\hline
\end{tabular}


Appendix 4:

Means and Standard Deviations for Boxing

\begin{tabular}{|c|c|c|c|c|c|c|c|}
\hline & & Day 1 & Day 2 & Day 3 & Day 4 & Day 5 & Overall \\
\hline \multirow{2}{*}{ SERT +/+ } & Mean & $0.00 \%$ & $0.00 \%$ & $0.09 \%$ & $0.08 \%$ & $0.01 \%$ & $0.04 \%$ \\
\hline & Standard Deviation & $0.00 \%$ & $0.00 \%$ & $0.05 \%$ & $0.05 \%$ & $0.01 \%$ & $0.02 \%$ \\
\hline \multirow{2}{*}{ SERT +/- } & Mean & $0.02 \%$ & $0.05 \%$ & $0.04 \%$ & $0.16 \%$ & $0.16 \%$ & $0.08 \%$ \\
\hline & Standard Deviation & $0.01 \%$ & $0.03 \%$ & $0.01 \%$ & $0.08 \%$ & $0.10 \%$ & $0.03 \%$ \\
\hline \multirow{2}{*}{ SERT -/- } & Mean & $0.00 \%$ & $0.00 \%$ & $0.00 \%$ & $0.00 \%$ & $0.00 \%$ & $0.00 \%$ \\
\hline & Standard Deviation & $0.00 \%$ & $0.00 \%$ & $0.00 \%$ & $0.00 \%$ & $0.00 \%$ & $0.00 \%$ \\
\hline
\end{tabular}

Appendix 5:

Means and Standard Deviations for Indirect Social Behaviour

\begin{tabular}{|c|c|c|c|c|c|c|c|}
\hline & & Day 1 & Day 2 & Day 3 & Day 4 & Day 5 & Overall \\
\hline \multirow{2}{*}{ SERT +/+ } & Mean & $6.68 \%$ & $6.42 \%$ & $4.06 \%$ & $5.82 \%$ & $5.34 \%$ & $5.66 \%$ \\
\hline & Standard Deviation & $0.48 \%$ & $0.55 \%$ & $0.60 \%$ & $0.81 \%$ & $0.47 \%$ & $0.46 \%$ \\
\hline \multirow{2}{*}{ SERT +/- } & Mean & $7.49 \%$ & $10.35 \%$ & $8.08 \%$ & $11.45 \%$ & $5.39 \%$ & $8.55 \%$ \\
\hline & Standard Deviation & $0.31 \%$ & $0.41 \%$ & $0.60 \%$ & $1.37 \%$ & $0.31 \%$ & $1.07 \%$ \\
\hline \multirow{2}{*}{ SERT -/- } & Mean & $8.44 \%$ & $9.61 \%$ & $5.33 \%$ & $7.85 \%$ & $5.62 \%$ & $7.37 \%$ \\
\hline & Standard Deviation & $1.52 \%$ & $1.39 \%$ & $0.76 \%$ & $1.09 \%$ & $0.91 \%$ & $0.83 \%$ \\
\hline
\end{tabular}

Appendix 6:

Means and Standard Deviations for Non-social behaviour

\begin{tabular}{|c|c|c|c|c|c|c|c|}
\hline & & Day 1 & Day 2 & Day 3 & Day 4 & Day 5 & Overall \\
\hline \multirow{2}{*}{ SERT +/+ } & Mean & $16.69 \%$ & $13.62 \%$ & $8.29 \%$ & $8.68 \%$ & $5.25 \%$ & $10.51 \%$ \\
\hline & Standard Deviation & $3.29 \%$ & $1.05 \%$ & $1.17 \%$ & $1.21 \%$ & $2.02 \%$ & $2.05 \%$ \\
\hline \multirow{2}{*}{ SERT +/- } & Mean & $12.59 \%$ & $8.74 \%$ & $6.49 \%$ & $7.18 \%$ & $4.66 \%$ & $7.93 \%$ \\
\hline & Standard Deviation & $2.38 \%$ & $0.39 \%$ & $1.19 \%$ & $0.62 \%$ & $1.33 \%$ & $1.34 \%$ \\
\hline \multirow{2}{*}{ SERT -/- } & Mean & $12.67 \%$ & $9.46 \%$ & $6.58 \%$ & $3.34 \%$ & $2.38 \%$ & $6.89 \%$ \\
\hline & Standard Deviation & $3.64 \%$ & $1.97 \%$ & $1.54 \%$ & $0.14 \%$ & $0.58 \%$ & $1.91 \%$ \\
\hline
\end{tabular}

Appendix 7:

Means and Standard Deviations for Social Grooming

\begin{tabular}{c|c|cccccc}
\hline \hline \multicolumn{2}{c}{} & \multicolumn{1}{c}{ Day 1 } & Day 2 & Day 3 & Day 4 & Day 5 & Overall \\
\hline \multirow{2}{*}{ SERT +/+ } & Mean & $21.92 \%$ & $18.52 \%$ & $21.75 \%$ & $15.96 \%$ & $19.69 \%$ & $19.57 \%$ \\
& Standard Deviation & $2.91 \%$ & $3.35 \%$ & $3.78 \%$ & $1.55 \%$ & $1.47 \%$ & $1.11 \%$ \\
\hline \multirow{2}{*}{ SERT +/- } & Mean & $25.34 \%$ & $22.33 \%$ & $17.77 \%$ & $16.09 \%$ & $8.20 \%$ & $17.95 \%$ \\
& Standard Deviation & $1.46 \%$ & $1.72 \%$ & $1.22 \%$ & $1.75 \%$ & $2.60 \%$ & $2.94 \%$ \\
\hline \multirow{2}{*}{ SERT -/- } & Mean & $30.64 \%$ & $25.34 \%$ & $23.62 \%$ & $25.72 \%$ & $24.62 \%$ & $25.99 \%$ \\
& Standard Deviation & $1.74 \%$ & $2.61 \%$ & $2.07 \%$ & $0.84 \%$ & $0.57 \%$ & $1.22 \%$ \\
\hline
\end{tabular}


Appendix 8:

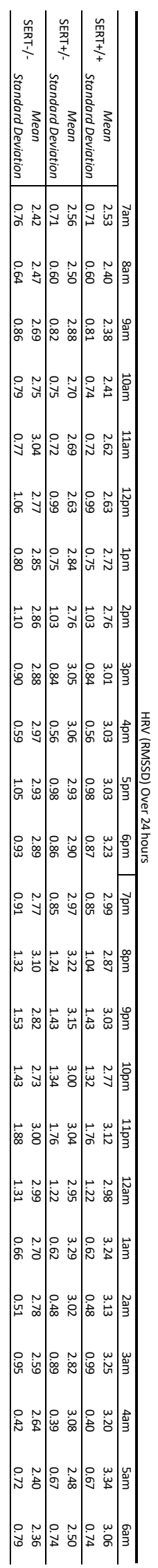


Appendix 9:

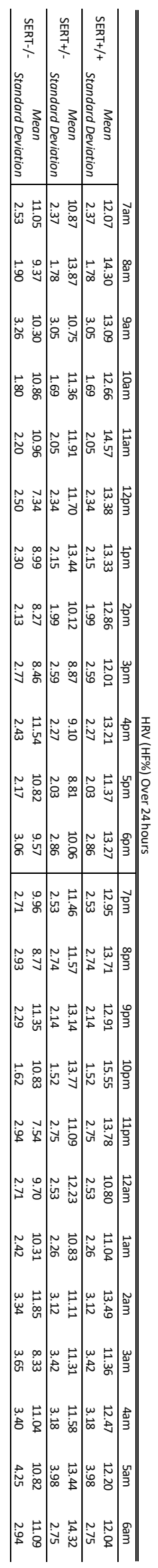


Appendix 10: Euthanasia and tissue extraction procedure

Animals via conscious decapitation in order to prevent hypoxia induced brain tissue deterioration and prevent neurochemical alterations caused by general anaesthetics. Brains were rapidly extracted within 45 seconds and snap frozen in liquid nitrogen to prevent degradation of neurotransmitters. Following the brain extraction, hearts were severed above the atria, weighed and snap frozen using the same method as the brain tissue. Following this, the telemetry device was extracted and weighed.

Appendix 11:

ECG Morphology: P and R Wave Heights

\begin{tabular}{c|rrrrrrr}
\hline \hline & \multicolumn{3}{c}{ High HRV } & & \multicolumn{3}{c}{ Low } \\
\hline & $\begin{array}{c}\text { P-Wave } \\
\text { Height }\end{array}$ & R-Wave & P/R & P-Wave & R-Wave & P/R \\
& 0.18 & 0.82 & 0.22 & 0.09 & 1.44 & 0.07 \\
Mean & 0.06 & 0.25 & 0.27 & 0.03 & 0.47 & 0.05 \\
\hline Standard Deviation
\end{tabular}

